\title{
Tolerant chimpanzee - quantifying costs and benefits of sociality in wild female bonobos (Pan paniscus)
}

\author{
Dissertation \\ for the award of the degree \\ “Doctor rerum naturalium” (Dr.rer.nat.) \\ of the Georg-August-Universität Göttingen
}

within the doctoral program Behavior and Cognition (BeCog)

of the University of Göttingen School of Science (GAUSS)

\author{
Submitted by \\ Niina Orvokki Nurmi \\ from Helsinki, Finland
}

Göttingen, 2018 


\section{Thesis Committee}

Prof. Dr. Hannes Rakoczy, Cognitive Psychology, University of Göttigen.

Dr. Dietmar Zinner, Cognitive Behavior, German Primate Center.

PD Dr. Oliver Schülke, Behavioral Ecology, University of Göttingen.

\section{Members of the Examination board}

Referee: PD Dr. Oliver Schülke, Behavioral Ecology, University of Göttingen.

Co-referee: Prof. Dr. Hannes Rakoczy, Cognitive Psychology, University of Göttigen.

Further members of the examination board

Prof. Dr. Julia Ostner, Behavioral Ecology, University of Göttigen

Prof. Dr. Eckhard W. Heymann, Behavioral Ecology and Sociobiology Unit, German Primate Center (DPZ), Leibniz Institute for Primate Research.

PD Dr. Matthias Waltert, Department of Systematic Conservation Biology, Johann-Friedrich-Blumenbach Institute of Zoology, University of Göttigen.

Dr. Dietmar Zinner, Cognitive Behavior, German Primate Center. 




\section{TABLE OF CONTENTS}

SUMMARY

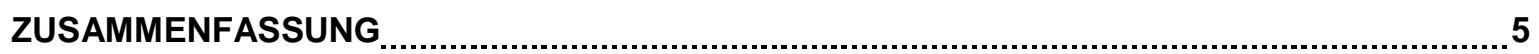

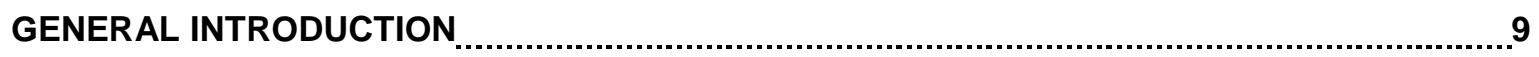

Socio-ecology in an evolutionary framework

Intrasexual selection

Competition for resources

Dominance hierarchies

Primate socio-ecology

Energy balance and allostatic load $\ldots$

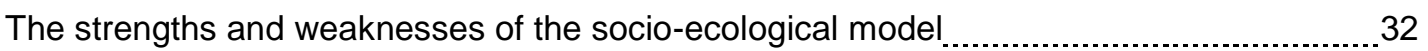

Study species

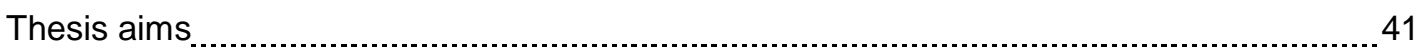

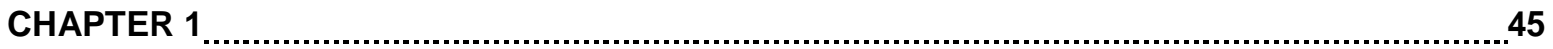

The "tolerant chimpanzee" - towards the costs and benefits of sociality in female bonobos. with Gottfried Hohmann, Lucas G. Goldstone, Tobias Deschner \& Oliver Schülke

CHAPTER 2

Effects of reproductive state and dominance status on urinary cortisol levels in wild female bonobos

with Gottfried Hohmann, Liza Moscovice, Tobias Deschner \& Oliver Schülke

GENERAL DISCUSSION

Summary of results

Mode of feeding competition in female bonobos

Effects of dominance rank on feeding efficiency but not on energy balance $\ldots . . \ldots \ldots \ldots \ldots \ldots . . . . . . .103$

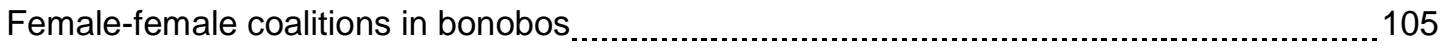

Effects of dominance rank and reproductive state on allostatic load $\ldots 107$

Conclusions and future directions

REFERENCES

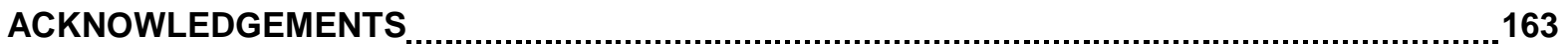

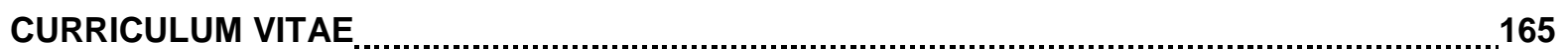

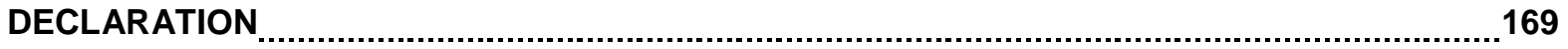







\section{SUMMARY}

Social interactions with conspecifics have fitness consequences for individuals. Socioecology is a subdiscipline of behavioral ecology. Socio-ecology integrates ecology, sexual selection, inclusive fitness theory, and evolutionary optimality modelling to advance our understanding of how social strategies evolve. Social strategies are sets of repeated individual behavioural decisions from which social systems eventually arise. A successful strategy maximizes an individuals' inclusive fitness. Behavioural decisions are constrained by several factors, many of which stem from the surrounding ecological and social environment. Group living comes with the costs of within-group competition for resources and increased disease transmission risk, which needs to balance out against the benefits of sociality for group living to evolve. Benefits of group living include reduced risk of predation, increased access to mates, defense of resources, and enhanced foraging success, among others. Social strategies are modulated by sex, because male and female fitness are limited by different resources. In mammals, female fitness is limited by access to food resources due to the energetic costs of gestation and lactation. Therefore, feeding competition tends to pose a greater cost of sociality for females than for males. Primate socio-ecology has focused on how food resource characteristics affect the distribution of females and their social strategies, which in turn defines the social strategies of males. Male coercion, infanticide risk, and predation risk are also key factors which shape female strategies.

Classical literature on resource competition makes a distinction between two main forms of competition, contest and scramble. Contest competition is a direct form of competition induced by clumped, defendable resources, and it results in a skewed distribution of resources among group members. Scramble is an indirect form of competition that results in equal resource distribution among group members but the amount received by everyone decreases with increasing group size. Primate socio-ecological models have been central in providing distinct predictions for the behavioural responses and net energy intake depending on the resource characteristics. The social outcomes describe the competitive regimes within and betw een groups, including characteristics of dominance relationships and degree of tolerance among females. The two species in genus $P a n$, chimpanzees ( $P$. troglodytes) and bonobos ( $P$. paniscus), share several social traits, including female dispersal and a high degree of fission-fusion dynamics. However, female bonobos are distinct due to their high gregariousness (i.e. high ratio of attendance in mix-sex parties and female cohesiveness), increased social tolerance and socio-sexual behaviours. The proximate mechanisms 
underlying these traits have not been studied rigorously thus far. Several hypotheses have been proposed to explain the species difference within the Genus Pan, however a formal test with the appropriate parameters has been lacking. As our closest living relatives together with the chimpanzees, studying bonobos in their natural habitat can also enhance our understanding of human evolution.

My thesis aim was test predictions derived from socio-ecological theory, which link repeated social interactions in feeding patches to variation in female energy balance and glucocorticoid levels reflecting allostatic load (i.e. the cumulative burden on the body due to environmental challenges) in female bonobos. In particular, my aim was to elucidate, whether the variation is consistently associated with female dominance rank position such that the costs of feeding competition are skewed. With my study, I was also able to comprehensively test a socio-ecological hypothesis, the Resource Abundance hypothesis, which proposes that differences in gregariousness between female chimpanzees and bonobos are due to the differences in the availability of abundant food resources. In addition, I tested two alternative hypotheses, the Cooperative Defence and Priority of Access hypotheses, proposed to explain the increased gregariousness in female bonobos specifically. The former proposes female defence of food resources against males as the main driver of female sociality in bonobos, while the latter invokes female defence of food resources against other females, respectively. To test the predictions for these hypotheses, I characterized the proximate mechanisms of within-group feeding competition in female bonobos by assessing the relative effects of social and ecological parameters on female feeding efficiency, energy balance and allostatic load. I collected data on 14 adult female bonobos of the Bompusa community at the study site of LuiKotale in Democratic Republic of Congo during two nine-month field seasons. I obtained data on food resource characteristics on a scale relevant to the bonobos from 683 feeding patches using the focal tree method. I recorded food intake rates and movement in focal trees to assess female feeding efficiency. I collected non-invasive urine samples to assess female energy balance based on C-peptide levels and allostatic load based on cortisol metabolite levels. I collected scan data on party composition and terrestrial herbaceous vegetation feeding, and used it to infer potential alternative female feeding strategies. To examine whether females predominantly formed coalitions against other females or against males, I analysed all occurrence data on agonistic interactions.

My first study evaluated the relative contributions of ecological and social factors on female feeding success, feeding effort and energy balance. Based on the combined effects 
of decreasing food intake and increasing movement in feeding patches, I established that bonobos depleted their food resources, which is a condition for feeding competition to occur. I found that high-ranking females had higher feeding efficiency than low-ranking females, because high dominance rank was associated with higher food intake rates in combination with lower probability of moving in feeding patches. This finding is consistent with withingroup contest competition and most likely results from high-ranking females attaining better feeding positions in food patches and avoidance of high-ranking females by low-ranking females. I found that variation in female energy balance was explained by monthly availability of fruits (positive association between energy balance and fruit availability), but not by female dominance rank. I demonstrated that low-ranking females did not compensate for the lower feeding efficiency by feeding in terrestrial herbaceous vegetation patches or by foraging in smaller parties than high-ranked females. I also found that female-female coalitions mainly targeted males. In combination with the patch depletion and lack of support for lower-ranking females using alternative feeding strategies, I have shown that the Resource Abundance hypothesis is an unlikely explanation for the differences in female gregariousness between chimpanzees and bonobos. My study lends stronger support for the Cooperative Defence hypothesis, suggesting that female gregariousness and tolerance in bonobos evolved due to the need of flexible coalition formation among the mostly unrelated females in defense of food resources against males and to deter harassment by males.

In my second study, I tested the predictions of the allostatic load framework to asses the effects of energetic condition, dominance status, and reproductive state on female cortisol metabolite levels as a proxy of allostatic load. I established, that there was no relationship between energy balance and cortisol levels measured from the same urine sample. I found that females in early lactation state had higher cortisol levels compared to females in cycling or late lactation state. I also found that the three highest-ranked females had higher cortisol levels compared to the 11 females on all other dominance rank positions. I also showed that females at the highest dominance rank position face increased allostatic load. Moreover, I found that early lactation increases allostatic load of females independent of dominance rank position.

The combined results of my study refine our understanding of female social relationships in a species that is peaceful and tolerant according to the widely held notion. Lower-ranking female bonobos suffer only limited costs of within-group feeding competition. Despite having lower feeding efficiency compared to high-ranking females, the energy 
balance of low-ranking females is similar to those of higher-ranked females. I did not find indication that low-ranked females used compensatory feeding strategies of feeding away from the main party or feeding more on terrestrial herbs. Another possibility is that lowerranking females feed longer, which I did not test for. However, I did find that highest-ranking females face costs of sociality that seem to be driven by social effects rather than by nutritional challenges. The increased costs of sociality to the highest-ranked female bonobos may be due to dominance rank maintenance and acquisition in the absence of support from female relatives on the one hand. Moreover, there may be additional energetic costs for those high-ranking females who provide agonistic support for their son(s). My study therefore consolidates the modulating effect of male strategies on female social relationships, and the importance of considering the synergistic effects of male and female strategies on sociality. 


\section{ZUSAMMENFASSUNG}

Die klassische Literatur über die Nahrungskonkurrenz unterscheidet zwischen zwei Hauptformen des Wettbewerbs: Interferenzkonkurrenz und Ausbeutungskonkurrenz. Interferenzkonkurrenz ist eine direkte Form des Wettbewerbs, die von gruppierten, verteidigbaren Ressourcen hervorgerufen wird und eine ungleiche Verteilung der Ressourcen zur Folge hat. Ausbeutungskonkurrenz ist eine indirekte Art des Wettbewerbs, die eine gleiche Verteilung der Ressourcen zu Folge hat, aber sich die Menge, die jeder bekommt, mit erhöhter Gruppengröße verringert.

Sozioökologische Modelle über Primaten bieten eindeutige Vorhersagen für Verhaltensantworten und die Energiebilanz abhängig von den Eigenschaften der Ressource. Die sozialen Auswirkungen beschreiben das Wettbewerbssystem innerhalb von Gruppen und zwischen Gruppen, wie Charakteristika der Dominanzbeziehungen und den Grad von Toleranz unter Weibchen. Die zwei Arten in der Gattung Pan, Schimpansen ( $P$. troglodytes) und Bonobos ( $P$. paniscus), haben einige soziale Eigenschaften gemeinsam, darunter die Abwanderung von Weibchen und starke Fission-Fusion-Dynamiken. Jedoch unterscheiden sich weibliche Bonobos von Schimpansen durch ihren hohen Grad an Geselligkeit, größere Toleranz und ihre sozio-sexuellen Verhaltensweisen. Bisher wurden die proximaten Mechanismen, die diesen Eigenschaften zugrunde liegen, noch nicht gründlich untersucht.

Meine Doktorarbeit hatte zum Ziel die Voraussagen, die von der sozioökologischen Theorie abgeleitet werden können, zu untersuchen. Diese stellt wiederholte soziale Interaktionen an Orten wo Nahrung vorkommt mit unterschiedlichen Energiebilanzen und allostatischer Belastung von weiblichen Bonobos in Bezug. Insbesondere war mein Ziel aufzuklären, ob diese Unterschiede durchweg mit der Position in der Rangordnung zusammenhängen. Mit meiner Studie konnte ich außerdem einen formalen Test der sozioökologischen Hypothese durchführen. Diese schlägt vor, dass Unterschiede in der Geselligkeit zwischen weiblichen Schimpansen und Bonobos durch die Verfügbarkeit üppiger Nahrungsressourcen im Lebensraum der Bonobos zustande kommen.

Des Weiteren untersuchte ich zwei alternative Hypothesen, welche besonders die erhöhte Geselligkeit von Bonobos erklären sollen. Um dies zu tun, beschrieb ich die proximaten Mechanismen der Nahrungskonkurrenz weiblicher Bonobos innerhalb von Gruppen, indem ich die relativen Auswirkungen sozialer und ökologischer Parameter auf die Effizienz bei der Nahrungsaufnahme, die Energiebilanz und die allostatische Belastung maß. 
Über zweimal neun Monate sammelte ich Daten von 14 weiblichen Bonobos der BompusaGruppe am Studienstandort LuiKotale in der Demokratischen Republik Kongo.

Mit der Fokusbaum-Methode (focal tree method) sammelte ich Daten über die Eigenschaften von Nahrungsressourcen, auf einer für Bonobos relevanten Skala, an 683 Orten an denen Nahrung vorkam. Ich erfasste die Rate der Nahrungsaufnahme und Bewegungen im Fokus stehenden Baum, um die Effizienz bei der Nahrungsaufnahme festzustellen. Ich sammelte nicht-invasive Urinproben, um die Energiebilanz von Weibchen anhand der C-Peptidmenge und der allostatischen Belastung (Menge der Kortisolmetaboliten) abzuschätzen. Ich sammelte Scan-Daten über die Zusammensetzung der Gruppe und das Fressen am Boden wachsender krautiger Vegetation und nutzte diese um mögliche unterschiedliche Strategien der Nahrungsaufnahme bei den Weibchen abzuleiten. Um zu untersuchen, ob Weibchen Koalitionen gegen andere Weibchen oder Männchen bilden, analysierte ich Daten über das Vorkommen agonistischer Interaktionen.

Meine erste Studie untersuchte die relativen Beiträge ökologischer und sozialer Faktoren zur erfolgreichen Nahrungsaufnahme, dem zur Nahrungsaufnahme nötigen Aufwand und der Energiebilanz bei Weibchen. Basierend auf dem Zusammenspiel geringerer Nahrungsaufnahme und erhöhter Bewegung innerhalb des Nahrungsvorkommens, konnte ich feststellen, dass Bonobos ihre Nahrungsressourcen erschöpfen, was eine Grundvoraussetzung für das Vorkommen von Nahrungskonkurrenz ist. Ich fand heraus, dass hochrangige Weibchen eine höhere Effizienz bei der Nahrungsaufnahme hatten als niedrigrangige Weibchen, da ein hoher Rang mit höheren Raten der Nahrungsaufnahme in Kombination mit einer geringeren Wahrscheinlichkeit der Bewegung innerhalb des Nahrungsvorkommens zusammenhing.

Ich fand heraus, dass Unterschiede in der Energiebilanz bei Weibchen durch die monatliche Verfügbarkeit von Früchten erklärt werden konnte, aber nicht mit der Position in der Rangordnung zusammenhingen. Ich zeigte, dass niedrigrangige Weibchen die niedrigere Effizienz bei der Nahrungsaufnahme weder dadurch kompensierten, dass sie krautige Vegetation am Boden fraßen, noch, dass sie in kleineren Gruppen auf Nahrungssuche gingen als höherrangige Weibchen. Ich konnte zeigen, dass Interferenzkonkurrenz ein Teil des Kokurrenzsystems von Bonobos ist. Dies kommt wahrscheinlich dadurch zustande, dass hochrangige Weibchen bessere Positionen für die Nahrungsaufnahme im Nahrungsvorkommen einnehmen und von niedrigrangigen Weibchen gemieden werden. Zusammen mit der Erschöpfung des Nahrungsvorkommens und in Ermangelung von 
Hinweisen darauf, dass niedrigrangige Weibchen alternative Strategien zur Nahrungsaufnahme wählen, habe ich gezeigt, dass die Hypothese des Überflusses an Ressourcen eine unwahrscheinliche Erklärung für die Unterschiede in der Geselligkeit zwischen weiblichen Schimpanse und Bonobos ist. So unterstützt meine Studie die Hypothese, dass sich die Geselligkeit und Duldung von Weibchen bei Bonobos evolutionär entwickelt hat, da größtenteils nicht-verwandte Weibchen flexible Koalitionen bilden müssen, um Nahrungsressourcen gegen Männchen zu verteidigen und Schikanen durch Männchen abzuwehren.

In meiner zweiten Studie untersuchte ich die Vorrausagen des Konzeptes der allostatischen Belastung, um die Wirkungen des energetischen Zustandes, der Position in der Rangordnung und des reproduktiven Zustandes auf die Menge an Kortisolmetaboliten bei den Weibchen, ein Maß für die allostatische Belastung, zu untersuchen. Ich stellte fest, dass es keine Beziehung zwischen der Energiebilanz und der in derselben Urinprobe gemessenen Kortisolmenge gab. Ich fand heraus, dass Weibchen in der frühen Phase der Milchbildung höhere Kortisolmengen hatten als sich im Zyklus befindende Weibchen und Weibchen in einer späteren Phase der Milchbildung. Ich fand auch heraus, dass die drei höchstrangigen Weibchen höhere Kortisolmengen aufwiesen, als die 11 Weibchen auf den anderen Positionen in der Rangordnung. Ich zeigte, dass Weibchen in den höheren Positionen in der Rangordnung einer größeren allostatischen Belastung ausgesetzt sind. Auch zeigte ich, dass die frühe Phase der Milchbildung die allostatische Belastung der Weibchen, unabhängig von der Position in der Rangfolge, erhöht.

Zusammengenommen verbessern die Ergebnisse meiner Studie unser Verständnis der sozialen Beziehungen zwischen Weibchen, bei einer Art, die allgemein als friedfertig und tolerant angesehen wird. Niedrigranginge Bonoboweibchen haben nur beschränkt Nachteile durch Nahrungskonkurrenz, da ihre Energiebilanzen ähnlich derer der hochrangingen Weibchen waren. Die höchstrangigen Weibchen sind durch die Sozialität benachteiligt. Dies scheint mehr durch soziale Ursachen, als durch Nachteile bei der Nahrungsaufnahme begründet. Die erhöhten Nachteile der Sozialität für weibliche Bonobos, könnten einerseits in der Erhaltung der Position in der Rangordnung und des Erlangens von Rang bei gleichzeitigem Fehlen von weiblichen Verwandten begründet liegen und andererseits durch ihre Beteiligung an Konflikten zwischen den Geschlechtern hervorgerufen werden, ins besonders wenn Unterstützung ihrer Söhne in Konflikten dabei eine Rolle spielt. 


\section{GENERAL INTRODUCTION}

\section{Socio-ecology in an evolutionary framework}

The fitness consequences of interactions between individuals are central to the understanding of the evolution of sociality. Natural selection is one of the most studied evolutionary processes because it generates adaptation based on the differential reproductive success of individuals (Gardner et al. 2011). Hamilton (1964) added another measure of reproductive success, inclusive fitness, to expand darwinian natural selection to the evolution of social behavior (Grafen 1985). Behavioural ecology is rooted in population genetics, yet the genetics that underlie social traits are often not known well enough or at all. Hence, behavioural ecology studies often start with the underlying assumption that individuals behave as if maximizing their inclusive fitness and that natural selection, in the present or at some time in the past, has caused them to behave in that way (Grafen 1984). Socio-ecology is a sub-discipline in behavioural ecology. Socio-ecology uses an evolutionary approach in order to understand the adaptive behavioural responses of individuals to varying selection pressures, with the ultimate aim of predicting the variation in social systems. In other words, a social system is an emergent property of individual behaviours.

A social system has the following three interrelated components: social organisation, mating system and social structure (Koenig et al. 2013). Following the definitions of Kappeler and van Schaik (2002), social organisation is a demographic component describing the size, cohesion and sexual composition of a social system. Individuals live alone (solitary), or with one (pair), or with several other individuals (groups) of the same or different sex (e.g. onemale multi-female, multi-male multi-female). Pairs and groups can vary in terms of temporal and spatial stability, ranging from cohesive to dispersed pairs or groups. Groups with fissionfusion dynamics form temporary subgroups that can vary in duration, size and composition, whereas multilevel groups are modular entities composed of smaller social units. Mating system is the component describing the degree of sexual conflict patterns of paternity distribution. It consists of monogamy (males/females mate with only one other individual of opposite sex), polyandry (one female mates with many males), polygyny (male mates with several females), polygynandry (both males and females mate with multiple partners), and promiscuity (several males mate with several females). Social structure is a component on an inter-individual level, describing variation in relationships within and between the sexes in terms of agonistic and affiliative interactions (Kappeler and van Schaik 2002). 
A typical socio-ecological (verbal) model uses the following principle; individuals make iterative behavioural decisions according to particular social strategies, these decisions accumulate to determine social relationships, which in turn form components of the social system (Wrangham 1979, van Schaik 1996). A strategy (see Cp. 7, Dawkins 1982) is a commonly used concept in evolutionary optimization models. Optimization models offer a way to understand adaptations by focusing on biological constraints (Parker and Maynard Smith 1990). If social strategies are adaptations, it then follows that those factors imposing the strongest constraints on individual fitness could also be expected to impose the strongest selection pressure on the evolution of social strategies (van Schaik 1996).

\section{Intrasexual selection}

Selection pressures may operate differently in males and females in sexually reproducing species. Bateman's (1948) seminal work on intra-sexual selection in fruits flies (Drosophila melanogaster) documented greater variance in the reproductive success of male flies as compared to female flies. He linked this sex difference to the mechanism of sexual reproduction with the following reasoning; male reproductive success was unlikely to be constrained by gamete production but rather by access to mates, whereas in most animals female reproductive success was likely to be constrained by gamete production, causing strain on energy acquisition. Moreover, in female mammals, the energetic demands would be further strained by gestation and lactation. Trivers (1972) extended this reasoning by proposing that the relative parental investment of the sexes determined the degree of intrasexual competition and the strength of sexual selection.

Later empirical research on food limited wild mammals has shown that the relative fitness costs of lactation are higher compared to gestation (Clutton-Brock et al. 1989). Clutton-Brock and Parker (1992) reformulated Trivers's concept by emphasizing the importance of potential reproductive rates of males and females and operational sex ratio (ratio of receptive males to receptive females at a given time; Emlen 1976) in shaping sexual selection. They also noted that the costs and benefits associated with the forms of competitive behaviour might differ between the sexes. However, Clutton-Brock and Parker's (1992) review adopts a narrower definition of sexual selection, as it focuses on intrasexual competition for mating partners. A broader definition would consider competition for reproduction in general, which would incorporate female-female competition for resources other than mates. Due to the traditional focus on competition for mates, the strength of sexual 
selection on females compared to males may have been underestimated (Stockley and BroJørgensen 2011).

Due to the lower potential reproductive rates of females, especially in mammals, females are expected to compete with females over access to resources, such as food and shelter, whereas males are expected to compete with males over access to mating partners. Therefore, females are expected to space themselves according to the distribution of resources in the environment, which then determines female social strategies depending on the costs and benefits of female-female association. The distribution of males and their social strategies is expected to depend on the dispersion of females. This line of reasoning is also at the heart of Emlen and Oring's (1977) influential paper on the evolution of mating systems, which posits that the spatial and temporal pattern of resource dispersion are the primary ecological determinants of the economical monopolizability of multiple mates (or the resources to attract multiple mates). The concept of economical monopolizability is a paraphrase of Brown's (1964) economical defendability, which reflects the balance between the costs (lost time and energy, risk of injury) and benefits (gained energy, mates or shelter) of defending the requisites for reproduction. When the benefits of defending resources outweighs the costs, territorial behaviour would be favoured as a social strategy. In other words, the economical defendability of resources may help in understanding what form of competition individuals might adopt in a given situation.

\section{Competition for resources}

Following the general definition of Miller (1967), competition is the demand by two or more individuals for a common (actually or potentially) limiting resource. The criterion of competition has been ambiguous at times, because there are empirical tests measuring the outcome of competition while others focus on the mechanistic processes and their components. Confusion has also ensued due to varying terminology and concepts used by different disciplines, or when competition is investigated at the intraspecific and interspecific levels (Birch 1957, Miller 1967, Parker 2000). There is a traditional distinction between two components of competition, namely exploitation and interference (Park 1954, Birch 1957). Exploitation is the independent use of a common resource by one individual without any regard to other individuals. Interference ensues when an individual prevents or tries to prevent other individuals from using a common resource (De Jong 1976). Exploitation and interference are sometimes used analogously with scramble and contest competition, 
respectively (e.g. Alatalo et al. 1987, Isbell 1991, Duijns and Piersma 2014), or treated as separate mechanisms occurring at different levels (De Jong 1976). Scramble and contest competition are concepts introduced by Nicholson (1954). He coined scramble to describe the way animals feeding on dead organic matter utilized their food resource, with the rate of food supply independent of the actions of animals. In contrast, contest was a type of competition deriving from the pattern of individual behaviours, with successful individuals excluding others from utilizing limiting resources, such as in territorial animals (Nicholson 1957).

From a density-dependent population growth perspective, scramble competition involves an equal partitioning of the resource such that when the resource becomes depleted, complete survival can switch rapidly to complete demise. In contest competition however, only the successful individuals secure a share of resources and hence as density increases, the number of survivors is expected to remain constant (Hassell 1975). While the implications of scramble and contest competition for population outcomes have been central for many ecological studies, socio-ecological studies have placed more emphasis on how the characteristics of limiting resources influence individual competitive strategies and spacing (Brown 1969, Brown and Orians 1970, Geist 1974, Jarman 1974, Bradbury and Vehrenkamp 1977).

Ideal free distribution is a concept born out the effort to understand how birds distribute themselves in relation to habitat distribution (Fretwell and Lucas 1970, Fretwell 1972). The model of an ideal free distribution depicts the theoretical distribution of competitors in a situation where basic habitat suitability depends mainly on food density and predation risk, and habitat suitability is expected to decrease with increasing competitor density. Key assumptions of the model are that individuals select a habitat best suited for their survival and reproduction (habitat selection is 'ideal'), and that individuals have equal competitive abilities so that no individual is able to prevent another from using the available resources (individuals are 'free' to enter any habitat). As the best habitats saturate with competitors, resource depletion leads to a situation where an individual settling on a lower quality habitat can achieve a similar rate of resource acquisition that it would have obtained from a better habitat. Hence, the average fitness of individuals is expected to be similar across all occupied habitats.

Fretwell and Lucas (1970) also introduce the concept of ideal dominance distribution as a hypothesis for predicting the distribution of territorial species. The model, also known as 
the ideal despotic distribution (Parker and Sutherland 1986), no longer has an assumption of a 'free' entry to a habitat. Instead, an individual arriving to the most suitable habitat first is expected to defend resources by establishing a territory, thus restricting the settlement of later arrivals. Later arriving individuals settle for lower quality habitats or are excluded from obtaining any resources (they become 'floaters' sensu Brown 1969) and hence the average fitness of the late arrivals is expected to be lower than the average fitness in the habitat. Although territoriality has received a lot of attention especially in avian studies, it represents only one manifestation of interference competition, whereas other examples include brief contests over single resource units or guarding ephemeral resources (Goldberg et al. 2001).

The assumption of equal competitive ability among individuals is unlikely to hold in most animal populations. Parker and Sutherland (1986) noted that tests of the ideal free model fell in to two types of situations according to the assumption concerning resource distribution, and that these types might mask an ideal free distribution with unequal competitors although the perceived situation appears like an ideal free distribution with equal competitors. The two types of situations are 'continuous-input' and 'interference'. Continuousinput is a situation where resource items (food or mates) arrive continuously to a patch and are utilized immediately, so that the number of competitors is expected to be proportional to the rate at which resource units are delivered to the patch. Hence, an increase in the number of competitors should also affect the average intake of resources in direct proportion (Parker 1970, Milinski 1979, Harper 1982). Because the negative effects result from resource depletion, the 'continuous-input' situation is also described as a type of exploitation competition (Tregenza et al. 1996). 'Interference' is a situation where resource densities vary to some extent and the presence of competitors hinders obtaining the resources efficiently so that individuals are expected to move to patches of lower resource density to avoid interactions with competitors (Goss-Custard et al. 1984). Because the negative effects arise from interactions between competitors, 'interference' is in direct reference to interference competition. Parker and Sutherland (1986) extended the ideal free distribution by considering three types of models for populations with competitive asymmetries among individuals that do not defend territories like in the ideal despotic model. These models included the continuous-input and the interference situations, and a third type modelled around kleptoparasitism interactions, where individuals steal resources from others (Brockmann and Barnard 1979, Ruxton and Moody 1997). 
Taken together, an individual might opt to compete for resources by scramble or by contest, leading to competitor distributions ranging from ideal free distribution to ideal despotic distribution. These are not necessarily strict categories (e.g. pure scramble type) but rather degrees of competitive strategies which can include both types, and individuals might switch between strategies at some point (Hassell 1975). The question naturally arises, what are the conditions under which individuals adopt a specific competitive strategy, and especially under what conditions might individuals opt for escalated physical fighting? This returns us to the insight of Brown (1964), namely that the decision to defend or not to defend resources is influenced by the spatial and temporal distribution of resources, by the density of competitors, and by the adaptive value of aggressiveness. Brown's concept represents a simple optimality model with a cost-benefit approach. Under energetic constraints, when the benefit of obtaining exclusive access to a resource such as food exceeds the costs associated to defense in terms of loss of time, energy, opportunities and risk of injury (from conspecific rivals but also predation due to reduced alertness), resource defense becomes economical due to a net benefit (Carpenter 1987). When competitor density increases, the costs of defending resources are expected to increase because more time might be spent in defense rather than utilisation. In contrast, as resources become more clumped spatially, the costs of defending are expected to decrease because an individual is able to confine their movement around a smaller area containing a higher resource density (Robb and Grant 1997). Spatial clumping can be defined statistically as the positive correlation between the mean and variance of resource density (Green 1979). Resource density and competitor density should be ideally considered together because they can have interacting effects on the costs of defense e.g. when the low costs of defending a clumped resource increase due to attracting more competitors (Vahl et al. 2005, Jacobson et al. 2015).

When there are asymmetries in competitive ability among individuals, spatial clumping is predicted to increase the monopolisation of resources as individuals of higher competitive ability shift from scramble competition to contest competition (Grant and Guha 1993). This prediction is largely supported by empirical studies using manipulations of resource distribution in fishes, birds and mammals (reviewed by Vahl et al. 2005). Divergent findings have been attributed to phylogenetic constraints (Ganslosser et al. 1997) or to the interacting effects of spatial and temporal clumping of resources (Robb and Grant 1998). Although the concept of economic defendability was originally conceived with territoriality in mind, it has been applied successfully in predicting the economic use of contest competition in non-territorial species and the strength of dominance hierarchies in social groups (Isbell 
1991). The dichtonomy of territorial and non-territorial behaviour is somewhat elusive, partly because of the way territory is defined in studies that have been conducted on different species under different contexts (Burt 1943, Kaufmann 1971).

There have also been propositions of treating territories and dominance hierarchies as the two poles of a behavioural continuum, governed by competitor density (Davis 1958). This approach, also known as the continuum of social organization theory, considers all resource related aggression such that the outcome of aggressive interactions is dependent on resource value and distribution, individual aggressive abilities, and competitor density. When the outcome of exclusive access to resources (i.e. 'territory' holding) is uneconomical, then partial access to resources can still be obtained using lower cost aggression (Craig and Douglas 1986). Although aggression is considered as one form of interference, contest competition might also manifest as displacements, subtle threats or avoidance behaviours (Shopland 1987). While aggression can be loosely defined as the behaviour of attack and threat (Deag 1977), the term agonism is used commonly to refer to the broader range of competitive behaviours including those involved in defense and retreating (King 1973). Aggression in particular may involve major costs for an individual and there is likely to be asymmetries in aggressive abilities among the competitors (e.g. due to age or residence status).

Game theoretical approaches applied in biology (Lewontin 1961, Maynard Smith and Price 1973, Maynard Smith and Parker 1976) have improved our understanding of competitive tactics, especially in terms of predicting the occurrence of escalated aggression. Maynard Smith and Price (1973) began with the premise that escalated conflicts among conspecifics are relatively rare and often involve ritualized conflicts or ineffective weapons. Their main point was to show that these 'limited war' type behaviours could be explained by individual selection without the need to invoke group selection (Maynard Smith 1979). Among their key insights is that the relative success of an individual adopting a specific strategy often depends on the strategies adopted by other individuals in the population and not just on the individuals own behaviour; in other words, fitnesses are frequency dependent. The HawkDove-Retaliator is an expanded game featuring three commonly modelled strategies; the Hawk escalates until victory or defeat, the Dove displays but retreats before being hurt if opponent escalates, and the Retaliator always displays on the first move but escalates if its opponent escalates (Maynard Smith 1982). The model aim is to find a strategy that would be stable under natural selection, in other words, an 'evolutionary stable strategy' (ESS). A 
strategy is considered to be an ESS if it is adopted by most individuals in a population, and rare individuals, which have adopted a different strategy, are unable to increase in frequency (Maynard Smith 1974).

In the expanded game, Hawks do well in a population mainly consisting of Doves, but rare Hawks can invade easily. Rare Doves can invade a majority Hawk population, depending on the costs of injury. Hence neither pure Hawk nor pure Dove is an ESS. While rare Hawks are not able to invade a population of Retaliators, rare Doves can invade and attain similar fitness to Retaliators, leading to a mixed population of Doves and Retaliators at equilibrium, albeit not as an ESS (McElreath 2007). The original simulations by Maynard Smith and Price (1973) illustrated how a 'limited war' strategy could be stable against a 'total war' (pure Hawk) or a 'total peace' strategy (Dove), when individuals began by using ritualized contest and escalated only when the opponent escalates. Some errors were pointed out in the early analysis however, which explains why the mix between Doves and Retaliators becomes selective neutral rather than an ESS (Gale and Eaves 1975, Maynard Smith 1982).

The early game theoretical models also worked under the assumption that the contest were symmetrical. Parker (1974a) noted that in natural situations contestants might assess the relative strength of their opponents before deciding to escalate, and that this might further depend on being a resource holder. He coined the term resource holding power as a measure of the absolute fighting ability of an individual, based on factors such as size, experience, strength and weaponry. Escalation is predicted only when the resource holding power of the contestants is closely matched. The odds are weighted towards the holder so that the attacker should have a higher relative resource holding power before escalating ('Bourgeois' strategy sensu Maynard Smith 1982). These developments led to the subsequent collaborative paper concerning asymmetric contests by Maynard Smith and Parker (1976). They split the analyses between correlated asymmetry, where fighting ability reflects ability to win, and uncorrelated asymmetry, where resource holding power is uncorrelated with the ability to win (see Grafen 1987 for a useful commentary concerning the 'Bourgeois' principle). The major conclusions are that mixed strategies are rare in asymmetric contests, and that the ESS is to permit an asymmetry cue (such as weapon size) in order to settle the contest without the need to escalate.

Individuals in social groups often experience repeated competitive interactions with their group members. Hence, it is likely that prior experiences of aggressive interactions, whether as a participant or as an observer, can influence the outcome of subsequent contests 
via so-called winner and loser effects, and bystander and audience effects (Chase et al. 1994, Dugatkin 2001). The winner effect can be defined as an increased probability of winning the subsequent contest upon winning the prior contest, and the loser effect similarly an increased probability to loose upon losing a prior contest (Chase et al. 1994). The bystander effect can be defined as a change in an observers' (i.e. the individual bystander) assessment of the contestants fighting abilities depending on the outcome of aggressive interactions, while the audience effects describes changes in fighting behaviour of a contestant when it is being observed by others (Dugatkin 2001). These effects are not considered in the game theoretical models described so far. Although the proximate mechanisms underlying winner and loser effects on fighting behaviour are not completely understood, there is ample indication that hormonal changes are involved. Winning fights increases plasma testosterone levels in territorial California mice (Peromyscus californicus) and the probability of winning a future aggressive encounter is significantly increased, independent of fighting ability (Oyegbile and Marler 2005). Loosing fights is linked to increases in plasma corticosterone levels in copperheads (Agkistrodon contortrix; Schuett et al. 1996). Winner and loser effects have received a lot of attention, especially in terms of their potential importance in structuring dominance hierarchies. Before proceeding to models that have incorporated there effects, it is useful to revisit some of the characteristics and concepts of dominance hierarchies.

\section{Dominance hierarchies}

Social dominance is an attribute of a pair of individuals based on the consistent outcome of agonistic interactions (Drews 1993). When a pair of individuals has repeated interactions trying to interfere with each other in securing a desired resource, the ability to link the competitive attributes of the individual and the previous outcomes can shift the conflict from aggressive competition towards lower intensity threat (Bernstein 1981). Hence, it is not always the case that the dominant status is assigned to a very aggressive individual because opponents can also show deference upon being attacked due to low chances of winning. While dominance status (dominant vs subordinate) is used to describe the status of an individual within the dyad, dominance rank reflects the arithmetic outcome of dominance interactions locating an individuals' relative position in a dominance hierarchy (Drews 1993). If a dominance hierarchy can be constructed in a given social group, it is often described in terms of its linearity (Landau 1951a), with linear or near-linear hierarchies being relatively rare and deviations from linearity more common e.g. one individual is dominant over all others but among the lower ranked individuals there are no individuals clearly dominating another 
one (Hinde 1978). Sometimes the dominance status of two individuals is unknown due to lack of interactions between them or due to sampling limitations, decreasing the linearity of the dominance hierarchy (Klass and Cords 2011). The dominance status can also be defined operationally as egalitarian, when neither of the two individuals wins dominance interactions consistently (Hand 1986).

Another characteristic of dominance hierarchies is the degree of their stability, which can be influenced by individual learning, ageing, health, stochastic processes and/or group composition (Hinde 1978). Depending on the pattern of dispersal, social groups often contain related individuals (Greenwood 1980). In mammals, including many primate species, it is more common for females to remain in their natal groups compared to males, which can lead to higher levels of relatedness among the group females (Dobson 1982, Pusey and Packer 1987). The increased chance to encounter a related individual in a dominance interaction can have a strong bearing on the stability of dominance hierarchies via coalitions with related individuals (Broom et al. 2009). Coalitions can also form among non-related individuals, such as bridging alliances targeting an individual between high and low rank (Chapais 1995).

It is worth noting however, that aggressive interactions can be as frequent or more frequent among related individuals, possibly due to increased proximity and increased opportunities for conflict (Bernstein 1988). However, conflicts among related opponents are often followed by a friendly reunion (reconciliation sensu de Waal and Yoshihara 1983) albeit the behaviors exchanged (e.g. allogrooming, gestures, restoration of proximity) can vary depending on the species (reviewed in Aureli et al. 2002). Therefore, some of the stressors stemming from the social environment, such as aggressive interactions, can be mitigated via social support from conspecifics (Creel et al. 2013). There is also a possibility that coalitionary support can affect morphological competitive traits, such as weaponry e.g. canine size tends to be smaller in primate species where females form coalitions (Plavcan et al. 1995).

Dominance hierarchies are often proposed to have a function in reducing aggression and risks of lost energy (Struhsaker 1967). However, a more parsimonious proposition is that predictable social relationships in general (independent of forming a hierarchical pattern) and a reduction in escalated contests ensues when individuals adopt social strategies that either maximize their fitness or minimize their losses when faced with a more formidable adversary in a contest situation (Deag 1977). As noted in the section concerning the forms of competition, the potentially interacting effects of resource holding power and winner/loser effects on dominance hierarchy had not been considered simultaneously in theoretical 
models. Landau (1951a, b) had already arrived at a conclusion that intrinsic factors (e.g. resource holding power) alone did not seem to produce linear hierarchies, whereas social factors (e.g. winner/loser effects) might better explain those animal societies with highly liner hierarchies. Dugatkin (1997) combined resource holding power and winner/loser effects in a single model to understand their simultaneous influence on dominance hierarchy formation. The model results reveal, that when contestants assess each other's resource holding power, the separate influence of winner effects produces dominance hierarchies with clearly assigned ranks of all individuals. In contrast, the separate influence of loser effects produces dominance hierarchies with a clearly distinct individual on the highest dominance rank position (alpha), but unclear dominance relations among all the other individuals due to scarcity of aggressive interactions. The loser effects are proposed to select for nonaggressive individuals, which means that most competitive interactions would result in both contestant deferring simultaneously (i.e. 'double kotow' sensu Dugatkin 1997).

The influence of bystander effects on dominance hierarchies has been modelled in a similar manner (Dugatkin 2001). When a bystander observers a contestant win, it raises its estimation concerning the contestants fighting ability (bystander winner effect), whereas when a bystander sees a contestant loose, it devalues the fighting ability correspondingly. Modeling of separate bystander winner effects produce social groups in which only the lowest dominance rank position (omega) is clear, whereas separate bystander looser effects produce groups without discernable dominance hierarchies.

One of the pervasive questions in terms of dominance hierarchies is what are the relative costs and benefits associated with various dominance rank positions? The question becomes particularly pertinent for studies investigating the correlation between dominance rank and reproductive success. Given that the benefit of high dominance rank position may often include priority of access to resources such as food or mates and fewer interruptions during feeding due to avoidance by lower-ranked individuals (primates: Whitten 1983, Wright et al. 2014; ungulates: Lovari and Rosto 1985, Thouless 1990), we would expect a positive correlation between dominance rank and reproductive success. A review by Ellis (1995) has found that males of many non-primate species fit the expectation, with high-ranking males having higher reproductive success compared to low-ranking males. The results for females of non-primate species are less clear, with some species showing a positive correlation while in others there is no significant correlation between dominance rank and reproductive success. The majority of findings on primate species suggest that high-ranking males have 
a strong reproductive advantage compared to low-ranking males. Number of males (i.e. male density) is an important source of variation in the relationship between dominance rank and reproductive success (Alberts et al. 2012). The results for female primates also show a clear positive relationship between dominance rank and female reproductive success, with a stronger signal seen in enhanced infant growth compared to infant survival (Pusey 2012). Harcourt's (1987) review on the correlation between dominance rank and fertility focuses on female primates. Based on three measures of fertility (age at menarche, birth rate, and timing of births), high-ranking females were significantly more fertile than low-ranking females. However, the correlation was not consistent across species or across years. There was ample indication that the effects of competition on fertility were due to food access, with stronger effects induced by spatially clumped foods (see also Harcourt 1989). One of the factors potentially blurring a correlation between dominance rank and fertility was differential investment depending on the sex of the offspring (Harcourt 1987).

Given, that there may be directional selection for high-ranking individuals to obtain greater share of fitness enhancing resources at the expense of low-ranking individuals, the question then arises what factors would keep the low-ranking individuals in the social group? Leaving the group may not always be a feasible option, as dispersing can entail risks ranging from increased predation risk in unfamiliar areas to hostility by new group members upon immigrating. On the other hand, dominant individuals might also have limitations in terms of using aggression, e.g. when the competitors are lower-ranking offspring or related individuals. Frequent aggression might also increase inter-individual distances, which might expose offspring or related individuals more readily to predators (Deag 1977). Therefore, the relative costs and benefits associated with various dominance rank positions also reflect the net benefits of group living in general, and the possible constraints on leaving a group.

Alexander's (1974) influential review on the evolution of social behaviour asserts that group living entails the automatic costs of competition for resources and mates, and greater risk of parasite transmission, but that these detriments can be overcome by the benefits of lowered predation pressure, grooming to remove external parasites, and group augmented localization of food resources or safe sleeping sites. Hence, social behaviour can evolve to enhance the benefits by facilitating cooperative hunting or defence of clumped resources, by reducing parasite transmission, and through its effects on reproductive competition among group members. The relative strength of those factors, along with options to leave the group, would then establish the limits to the degree to which high-rankers can monopolize resource 
without the risk of losing group members, which consequently secure a lower share, but might be needed for social support or an extra pair of eyes to spot predators (Vehrencamp 1983).

\section{Primate socio-ecology}

Primates have a flexibility of social systems proposed to be a consequence of variation in ecological conditions (Crook and Gartlan 1966). The initial approach by Crook and Gartlan used broader ecological grades (such as habitat types) in predicting primate social organization. Their contribution was influential in guiding future studies, but one of the shortcomings was that the categories made it difficult to pinpoint which selection pressures were operating (Janson 2000). Denham (1971) offered an alternative model that highlighted the importance of energy acquisition and anti-predator behaviour. Another shortcoming of the categorical approach by Crook and Gatland was that it could not address variation between species within the grades. As comparative field studies accumulated, sympatric, closely related primate species often differed in group size and hence in social structure, and these differences were linked to temporal and spatial clumping of food resources (e.g. colobines: Clutton-Brock 1974; macaques: MacKinnon and MacKinnon 1978; lemurs: Sussman 1974). Primate socio-ecology started around this time to have a more refined focus on how the distribution of food and risks in the environment shaped social strategies and social relationships, especially among females (Wrangham 1979, Wrangham 1980, van Schaik 1989). The role of social factors, including male harassment of females (Wrangham 1979, Brereton 1995) and infanticide risk (van Schaik 1996, Sterck et al. 1997), have also been emphasized.

Wrangham (1979) proposed that food distribution was the main ecological factor determining the social system of apes because it was the most cost-effective resource in terms of increasing female fitness. Predation received less attention due to the low perceived risk of predation on great apes. He reasoned that female apes are expected to forage without other females to minimize the costs of feeding competition, unless they mainly fed on widely available dispersed foods leading to lower costs of competition. Indeed, females of most ape species, including orangutans, gibbons, siamangs and eastern chimpanzees, tend to spend considerable time away from other females. Hence, food dispersion appears to define the competitive strategies and spatial distribution of female apes, which in turn influences male ape competitive strategies. Wrangham (1979) noted that male strategies can modulate 
female strategies due to social factors such as male harassment of females, and hence the social systems eventually depended on the distribution of both sexes.

However, most diurnal primate taxa show female gregariousness (van Schaik 1996), calling for a better understanding of the adaptive significance of social groups. Wrangham (1980) presented an influential ecological (verbal) model for the evolution of female-bonded groups. By female-bonded groups, he referred to stable groups based around a core of related, resident females. He posed the common occurrence of female-bonded groups as a paradox, because the costs of competing with relatives for food are expected to select for female dispersal rather than philopatry. Wrangham suggested that female-bonded groups evolved because the costs of feeding competition within groups was outweighed by the benefits of female-female cooperation in defending food resources against other groups containing less related females. Cooperation would only be effective, when food resources were defendable i.e. they were found in high quality patches, and when food patch size were large but contained limited number of feeding sites. These ecological conditions seemed to fit particularly well to frugivorous primates, many of which also lived in female-bonded groups. As for non-female-bonded groups, Wrangham predicted that such species would not feed on food occurring in high-quality patches.

Van Schaik (1983) tested Wrangham's model against an alternative hypothesis, namely that group living in primates evolved due to benefits accrued from protection from predators. Van Schaik compared data on fecundity and juvenile survival in groups of varying sizes and found that fecundity did not increase with group size and juvenile survival was higher in groups habiting areas with natural predators. He concluded that predation pressure was likely to determine the minimum size of groups, whereas feeding competition within groups would determine the maximum group size in primates. According to this view, the optimal primate group size can be modeled as an evolutionary compromise between the benefit of predator protection and the cost of within group feeding competition (Terborgh and Janson 1986).

An example of the functional link between group size and social systems is species with fission-fusion dynamics. Individuals in such groups can respond to varying food patch sizes because groups split in to smaller, temporary sub-groups of varying composition and duration. At the same time, foraging in small groups also enhances the risk of predation so that fission-fusion dynamics could be expected to be found in species that have a large body size and few predators (Terborgh and Janson 1986). Most mammalian species that are 
considered to exhibit relatively high degrees of fission-fusion dynamics are indeed relatively large in body size, including bottle nosed dolphins (Tursiops spp.), African elephants (Loxodonta africana), spotted hyenas (Crocuta crocuta), spider monkeys (Ateles spp.), chimpanzees (Pan troglodytes) and bonobos (Pan paniscus) (Asensio et al. 2008). Terborgh and Janson (1986) reminded however, that understanding the factors shaping group characteristics such as size could only have limited value in explaining social systems. Instead, the impact on individual fitness would need to take precedence because this was also the level at which natural selection was operating on. They advocated the use of quantitative methods in determining the extent to which social effects, such as reduction in food intake due to competition within and between groups, affected individual fitness. These guidelines provided an impetus to provide standardized methods to quantify feeding competition.

The two traditionally recognized forms of competition, contest and scramble, have different implications for food intake. Janson and van Schaik (1988) devised four types of competition for group living species: within-group contest competition, within-group scramble competition, between-group contest, and between-group scramble competition. They built a model to predict how each type would predict individual food intake depending on group size and an individuals' dominance rank. Between-group scramble was not included as it was not expected to affect the costs and benefits of group living. According to the model, the importance of within-group contest can be separated from within-group scramble and between-group contest because only the former causes variation in food intake among individuals within groups. Within-group scramble competition is sensitive to group size such that individuals, independent of dominance rank, have reduced food intake in larger groups. Between-group contest is also sensitive to group size such that individuals have increased food intake in larger groups. The interaction of contest and scramble competition within groups yields equal individual food intake in smaller groups but increasingly steeper dominance rank-dependent variation in food intake as the groups get larger (Janson and van Schaik 1988).

One of the limitations of the ecological model of female-bonded groups by Wrangham was the sole focus on contest competition between groups without considering scramble effects. Van Schaik (1989) extended Wrangham's (1980) ecological model of female-bonded groups by including within-group contest and within-group scramble. In addition, he included predation risk as an additional factor favouring cohesive social groups. He characterized the 
ecological conditions for different types of competitive regimes. Within-group contest is predicted when food occurs in clumped patches that are too small to accommodate all group members. Within-group scramble is predicted when food occurs in dispersed patches that are large enough to fit all group members. In both cases, groups are expected to be cohesive due to high vulnerability to predators. Between-group contest is expected when foods occur in large defendable patches, and groups are expected to be flexible due to lower predation risk either stemming from large body size or lack of natural predators. Van Schaik (1989) also characterized the expected female social relationship under each competitive regime. Dominance rank can enhance food intake under within-group contest conditions and hence dominance hierarchies are expected to be linear and nepotistic, with maternal support of relatives increasing rank stability. In contrast, under within-group scramble conditions dominance status does not enhance food intake due to the resource characteristics, and hence dominance hierarchies are expected to be weakly differentiated and non-linear, with limited advantages of supporting relatives decreasing rank stability. Van Schaik (1989) noted that although groups could be more or less cohesive under between-group contest conditions, contest within groups would be inevitable and hence subordinates might be tempted to leave a group or just parasitise on the food resources without helping in a contest between groups. He therefore predicted that under strong between-group contest, dominance hierarchies would be tolerant because subordinate females would have more leverage on high-ranking females. However, dominance hierarchies would also be nepotistic, because females would ally with relatives for increased stability and inclusive fitness benefits, as suggested by Wrangham (1980).

Besides the characteristics of dominance relationships, competition for food can be inferred from the following observations: female-female aggression between groups (ringtailed lemurs: Pride 2005; vervets: Cheney and Seyfarth 1987), increasing home-range size with increasing group size (Japanese macaques: Takasaki 1981), longer day range with increasing group size (capuchins: De Ruiter 1986; mangabeys: Olupot et al. 1994; spider monkeys: Symington 1988) and lower reproductive rates in larger group (leaf monkeys: Borries et al. 2008; black howler monkeys: Van Belle and Estrada 2008). An examination of daily ranging and patterns of female aggression in 20 primate species by Isbell (1991) found general support for Wragham's model of cooperative defense of resources. As a deviation from Wrangham's model however, she proposed that food distribution influences only withingroup competition whereas food abundance influences between-group contest. Isbell (1991) expanded the two types of female relationships proposed by Wrangham (1980), 
resident/differentiated (female-bonded) and dispersing/non-differentiated (nonfemalebonded), by adding a third type: resident/non-differentiated. She concluded, that alternative explanations, such as infanticide risk or male harassment, might better explain lower reproductive rates of females in larger groups than feeding competition effects.

In a review on primate socio-ecology, van Schaik (1996) noted that social factors such as male strategies and male-female association had been ignored from models explaining female relationships based on mainly ecological factors. The proposition of males as protectors against conspecific infanticide or harassment threat had been advocated previously (Wrangham 1979, Smuts and Smuts 1993). Observations of infanticide were cumulating (e.g. savannah baboon: Collins et al. 1984; Hanuman langur: Newton 1988; ringtailed lemur: Hood 1994), although its evolutionary importance had been debated for some time (Bartlett et al. 1993, Sussman et al. 1995).

Van Schaik (1996) foreshadowed the transition of the ecological model in to a socioecological model of female social relationships by Sterck et al. (1997). The synthesis by Sterck et al. (1997) posits that predation risk, infanticide risk and food resource distribution are the main factors underlying evolution of social systems. The basic premise, that withingroup contest competition occurs when food resources are clumped and of high quality has been retained from the earlier models. Female agonistic relationships are elaborated using three dimensions: egalitarian to despotic (sensu Vehrencamp 1983), individualistic to nepotistic (sensu Hrdy and Hrdy 1976), and varying levels of tolerance (sensu de Waal 1989). As a summary, Sterck et al. (1997) make a distinction between four social categories. The category Resident-Nepotistic is expected under strong within-group contest combined with no/low between-group contest; females should be philopatric and form stable alliances with relatives leading to linear, despotic dominance hierarchies. Dispersal-Egalitarian is expected under weak within-group and between-group contest competition; females should be 'free' to disperse because coalitions are not beneficial, dispersal may still depend on social risks such as infanticide. Resident-Egalitarian is a category predicted under the conditions of strong between-group contest combined with weak within-group contest; although coalitionary support of relatives is not required for decided dominance interactions within groups, female philopatry is expected because females are less likely to withhold their support during contests between groups. Finally, Resident-Nepotistic-Tolerant is expected under strong within-group and between-group contest; females should be philopatric and form stable linear dominance hierarchies, however, higher ranking females are predicted to show tolerance 
towards lower rankers so that the former would not risk losing cooperative support of the latter due to lack of incentive.

The socio-ecological model sensu van Schaik 1989 and Sterck et al. 1997 aims at connecting the causal factor of food resource characteristics to i) the proximate mechanisms of feeding competition (i.e. competitive regimes), ii) the ultimate effects on female fitness due to feeding competition, and iii) systematic variation in female social relationships and dispersal patterns (Koenig 2000). From an evolutionary perspective, female relationships are thought to reflect social strategies for maximizing fitness. One of the main strengths of the socio-ecological model is therefore the testable predictions concerning the ultimate effects in terms of net energy gain or reproductive success of females as fitness proxies.

\section{Energy balance and allostatic load}

Energy obtained from food resources in the environment is allocated between several processes that promote survival and enhance long-term reproductive success (Wade and Schneider 1992). Life histories reflect the strategies according to which organism prioritise energy allocation between maintenance functions, growth, and reproductive effort (Ellison 2003). Energy availability in natural environments is rarely constant because food abundance can fluctuate both temporally and spatially. Energetic demands of individuals can also vary with physiological states such as reproductive cycles or age. These aspects are particularly relevant for female mammals because of the energetic constraints of lactation and gestation (Clutton-Brock et al. 1989). As the body size of a mammal increases, its energetic constraints changes (Kleiber 1975, Bronson 1989). Larger mammals require relatively less food for maintenance and are also able to store more energy compared to small mammals (Gittleman and Thompson 1988).

Primates have been shown to expend only half the amount of energy that would be expected for a mammal of similar weight, and hence the slow metabolic rate may be one explanation for the slow life histories of primates (Pontzer et al. 2013). The endocrine system is an important mediator of energy allocation and hence considered as major regulator of life histories (Finch and Rose 1995, Dantzer et al. 2016). Energy status is the stored energy in an individual available for mobilization towards its reproductive effort. Energy balance is the net difference between energy intake and energy expenditure. An individual in high energy status is considered to have recently been in a state of positive energy balance, and both energy status and energy balance are expected to correlate positively with energy available 
for allocation to reproduction (Ellison 2003). However, an individual can also be in negative energy balance while having high energy status, when it has significant at stores but is losing weight nonetheless (Emery Thompson 2017).

Energy intake is a measure of the calories that can be absorbed from ingested foods (Emery Thompson 2017). Energy intake is largely determined by environmental factors such as food availability and abundance, but also by social factors such as group size or social status (Wilson et al. 2014). Energy intake can be approximated by measuring feeding effort (i.e. the time spent feeding) but using this measure alone may lead to spurious inferences concerning food intake and ultimately energy intake (Zinner 1999, Schülke et al. 2006). The measurement of food intake rates during behavioural observation in combination with analysis of chemical composition of food items for nutritional value can yield a more accurate measure of energy intake (Emery Thompson 2017). Some of the drawbacks are that obtaining accurate measurements of food intake rate can be challenging in field conditions (Nakagawa 2009), and the measurements of chemical composition might ignore variation within the same food patch (Houle et al. 2014).

Energy expenditure is a measure of the calories used through all metabolic pathways (Ellison 2017). These pathways relate to expenses due to resting metabolic rate, immune defenses, gestation, lactation and physical activity. The portion of expenditure on physical activity can be measured as distance (or time) traveled during the day (e.g. Pontzer et al. 2004), whereas measuring the proportion of energy expenditure devoted to immune defenses or lactation is more challenging (Emery Thompson 2017).

Direct physiological measures of energy balance based on insulin secretion offer an alternative approach to calculations of energy intake and expenditure rates. Insulin is one of the hormones that regulates metabolism. The $\beta$-cells of the pancreas produce and secrete insulin in response to changes in the extracellular glucose concentrations (Hedeskov 1980). Insulin facilitates energy uptake into target organs, including liver, adipose, and muscle tissue (Belgardt and Stoffel 2014). During lactation, changes in insulin sensitivity direct energy allocation towards milk production and these shifts in energy allocation correlate with lower insulin levels in humans (Ellison and Valeggia 2003). The biosynthesis of insulin involves an initial, larger precursor, preproinsulin, which is converted rapidly in to proinsulin. Proinsulin is further converted in to the polypeptide chain insulin and the polypeptide C-peptide within secretory granules of the $\beta$-cells, where they are both found in equal amounts (Bonser et al. 1984). Unlike insulin, C-peptide is excreted in the urine with a consistent fraction of $\sim 5$ percent 
(Kuzuya et al. 1976, Sherry and Ellison 2007). Urinary C-peptide is often indexed by creatinine to correct for differences in urine concentration (Taussky et al. 1954) or alternatively by specific gravity (Pradella et al. 1988, Anestis et al. 2009). Following the 1975 discovery of C-peptide in urine by Kaneko and colleagues (Kaneko et al. 1975), it has been an important biomarker for insulin production in clinical studies of diabetes and obesity.

At a later stage, urinary C-peptide has been established and validated as a noninvasive biomarker of energy balance in primate species, including captive chimpanzees (Pan troglodytes) and wild orangutans (Pongo pygmaeus) by Sherry and Ellison (2007). It has also been validated based on experimental weight change in captive bonobos ( $P$ an paniscus; Deschner et al. 2008) and captive macaques (Macaca mulatta, M. fascicularis: Girard-Buttoz et al. 2011). Several field studies on primate behaviour, energetics and ecology have used urinary C-peptide as a metabolic marker of energy balance in a range of species, including guereza (Colobus guereza; Harris et al. 2010), Sanje mangabey (Cercocebus sanjei; McCabe and Emery Thompson 2013), mantled howler (Alouatta palliata; CanoHuertes et al. 2017), chimpanzee (Pan troglodytes; Emery Thompson et al. 2009, Emery Thompson et al. 2012, Emery Thompson et al. 2014), bonobos (Pan paniscus; Georgiev et al. 2011, Surbeck et al. 2015), orangutan (Pongo pygmaeus; Emery Thompson and Knott 2008), and mountain gorilla (Gorilla beringei beringei; Grueter et al. 2014). Feeding on preferred foods has been found to increase female C-peptide levels in white-faced capuchins (Bergstrom 2015), mountain gorillas (Grueter et al. 2014), and chimpanzees (Emery Thompson et al. 2014). Because of the association between altered insulin production and lactation, C-peptide has the additional benefit of providing information about the costs of lactation. In lactating chimpanzees, urinary C-peptide levels remain stable for the first six months of lactation followed by a sudden increase (Emery Thompson et al. 2012). A similar pattern of low urinary C-peptide levels after parturition have been documented in humans, albeit with a more gradual increase of C-peptide during the first year post-partum (Ellison and Valeggia 2003). Some authors have also cautioned, that in humans C-peptide can only explain 30 percent of variation in energy balance (Bergouignan et al. 2012). Dominance rank is predicted to enhance access to food resources under within-group contest competition and therefore higher-ranking females are predicted to have higher C-peptide levels when contest competition prevails. Studies of baboons (Lodge 2012), capuchins (Bergstrom 2015), and mountain gorillas (Grueter et al. 2014) did not find a relationship between dominance rank and urinary C-peptide levels of females. In contrast, higher urinary C-peptide levels have been found in high-ranking male bonobos when travelling in large parties (Surbeck et al. 
2015), whereas high-ranking male chimpanzees have lower C-peptide levels than lowranking males (Emery Thompson et al. 2009). Urinary C-peptide thus provides a useful fitness proxy to enhance our understanding of the social consequences of feeding competition because it yields an integrated view of not only energy intake but also energy utilization and allocation to reproduction.

An alternative view to the social consequences of feeding competition can be obtained by incorporating endocrinological data linked to the vertebrate stress response. Challenges posed by the external environment provide sensory stimuli that initiate a neuroendocrine cascade involving parts of the hypothalamic-pituitary adrenal axis (Creel et al. 2013). Stressors are sensory stimuli that pose a direct threat or a perceived as threatening, to an individual's homeostasis (Boonstra 2013). Homeostasis refers to the stability of physiological systems that are considered essential for sustaining life (McEwen and Wingfield 2003). Stressors can derive from external sources including ecological conditions (perturbations in food availability, weather etc.) and the social environment (challenges due feeding competition or conspecific threats) (Beehner and Bergman 2017). Internal stressors stem from stimuli due to changes in life history stage or reproductive state, and can be quite predictable (Boonstra 2013). External stressors can often be unpredictable, such as predation events or attacks by conspecifics.

Stressors can be divided into two classes, reactive and anticipatory (Herman et al. 2003). Reactive stressors are direct threats to homeostasis and do not necessitate cognitive input, whereas anticipatory stressors are posed by cues that the individual perceives as potentially threatening and hence require some degree of cognitive processing (Boonstra 2013). These two classes are also known as physiological and psychosocial stressors sensu Sapolsky (2005). Stressors can also be classified based on duration as acute (minutes, hours) or chronic (days, weeks); however, the duration should be defined by the consequences on physiology rather than by the stressor (Boonstra 2013).

The hypothalamic-pituitary-adrenal axis is one of the main anatomical structures that mediate the vertebrate stress response. Together with other structures, such as the parasympathetic systems and noradrenergic neurons of the brain stem, the hypothalamicpituitary-adrenal axis regulates adaptive responses to homeostatic challenges (Smith and Vale 2006). The activation of the hypothalamic-pituitary-adrenal axis culminates in the release of glucocorticoids, which in turn can inhibit the axis through a negative feedback-loop (Smith and Vale 2006, Dantzer et al. 2016). Glucocorticoids are metabolic steroid hormones 
released from the adrenal glands upon stimulation by adrenocorticotropic hormone (Beehner and Bergman 2017). The two primary glucocorticoids produced are cortisol and corticosterone, and their predominance depends on the species (Koren et al. 2012).

Glucocorticoids are not only involved in the vertebrate stress response, as they are also important mediators of more basic metabolic functions such as circadian rhythmicity and digestion (Beehner and Bergman 2017). Glucocorticoids and insulin are reciprocally involved in signaling to the brain leading to regulation of feeding behaviour; hence both are involved in the long-term regulation of energy balance (Strack et al. 1995, Dallman et al. 2007). Upon a stress response, one of the preeminent metabolic effects of glucocorticoids is to increase glucose concentrations in blood circulation. This is achieved via inhibition of insulinstimulated glucose uptake and permission of catecholamine-induced breakdown of glycogen, which is a storage form of carbohydrates in mammals (Jensen et al. 2011, Kuo et al. 2013). In addition, glucocorticoids inhibit costly processes such as growth and reproduction, and mediate a cognitive stress response at the onset of a stressor by facilitating memory consolidation and retrieval, which can help in avoiding similar future stressors (Sapolsky 2000). Glucocorticoids are metabolized mainly in the liver after which they are excreted as conjugates in faeces and urine (Palme et al. 2005). Following the development of methods to measure urinary and faecal glucocorticoid metabolites from non-invasive samples collected in the field, several studies have investigated effects of different stressors in wild ranging animals (Touman and Palme 2005, Creel et al. 2013).

The interpretation of environmental data collected in natural condition can be challenging due to the varying contexts of stressors. A stressor might induce differential glucocorticoid release depending on the social context, food availability, or reproductive state for example (Romero 2004). In social groups of primates, individual differences in stress responses may reflect the dominance style of the species. In other words, it is important to understand whether individuals can monopolise access to resources (e.g. highly skewed access in despotic hierarchies), are on the receiving end of aggressive interactions, or have the chance to establish predictable and stable social relationships (Abbot et al. 2003, Sapolsky 2005). Coping mechanisms such as the presence of related individuals, reconciliations after conflicts and the ability to fission to join a smaller sub-group can buffer responses to stressors especially for subordinate individuals (Sapolsky 2005). Because of the negative relationship between net energy intake and dominance rank position under within-group contest competition, subordinate females might be more prone for internal 
stressors stemming from restricted access to nutrients. Moreover, these effects may be enhanced during energetically challenging internal states, such as lactation.

Based on a number of empirical studies, there does not seem to be a clear relationship between dominance rank position and endocrine profile among primate species, perhaps partially in reflection of the diversity of the social systems (Abbot et al. 2003). However, some of the patterns indicate a trend for higher glucocorticoid levels in relation to the dominance rank position that entails greater metabolic or energetic demands, either due to lower energy intake or increased energy expenditure (Beehner and Bergman 2017). Another pattern concerns a sex difference; the majority of studies on males show a positive relationship between dominance rank and glucocorticoid levels, whereas the majority of studies on females show a negative relationship. This seems to indicate, that glucocorticoid secretion depends on how dominance rank position is obtained, rather than being a high or low ranked individual in general (Beehner and Bergman 2017). The relation between dominance rank status and glucocorticoid levels can be quantified using the allostatic load framework (Goymann and Wingfield 2004). Allostasis is a process that supports the maintenance of homeostasis (McEwen and Wingfield 2003). Allostatic load is the cumulative wear and tear of the body due to adapting to the physiological stressors such as changing season or reproductive state (McEwen 1998). The increase in allostatic load reflects increasing energetic demands (McEwen and Wingfield 2003). Allostatic overload is a state where additional unpredictable loads such as injury or unstable social environment exacerbate the existing allostatic load (Goymann and Wingfield 2004).

As the main mediators of allostasis, glucocorticoid metabolite levels provide a proxy measure of allostatic load and allostatic overload (McEwen and Wingfield 2003). The allostatic load framework emphasises the mechanism by which dominance rank position is acquired and maintained (Goymann and Wingfield 2004). The costs of obtaining a high rank position are expected to be low for nepotistic dominance hierarchies, or if dominance rank is attained through queuing conventions, such as in African elephants (Loxodonta africana; Archie et al. 2006), chimpanzees (Pan troglodytes schweinfurthii; Foerster et al. 2016), and gorillas (Gorilla beringei beringei; Robbins et al. 2005). The costs of obtaining high rank are expected to be moderate when dominance rank is acquired using low level threats or overt aggression e.g. when the hierarchy decreases with age as in Hanuman langurs (Presbytis entellus; Borries et al. 1991) or howler monkeys (Alouatta palliata; Zucker and Clarke 1998). The costs of dominance rank maintenance are expected to vary with hierarchy stability. Given 
the relatively high stability of nepotistic and age-graded hierarchies, high rank is expected to correspond to low levels of allostatic load. In contrast, high levels of allostatic load in dominant individuals are expected in groups where the use of overt aggression by dominants is frequent and subordinates often challenge dominants (Goymann and Wingfield 2004). Incorporating measures of urinary glucocorticoid levels can yield valuable information concerning female dominance style and social relationships, including the costs of rank maintenance and acquisition, and the availability of support mechanisms for low-ranked females (Schülke and Ostner 2012).

\section{The strengths and weaknesses of the socio-ecological model}

Several studies have tested predictions of the socio-ecological model, although no studies have provided a full test of it thus far (Schülke and Ostner 2012). The majority of the tests seem to provide support for the models general principle linking food resource characteristics to female social relationships. Some of the exemplary tests are comparisons between two closely related species, or between populations of the same species, that differ in some social aspect. Mitchell et al. (1991) compared two taxonomically close, yet socially distinct, species of squirrel monkeys (Saimiri sciurus and S. oerstedi) to examine whether food distribution, feeding aggression or between-group interactions explained some of the observed social differences. They found that the female-bonded S. sciureus fed more often on defensible fruit patches compared to the non-female bonded S. oerstedi, which mostly fed on tiny fruits in patches that were most likely too small to be worth defending. Due to the limited indication for between-group competition yet relatively high predation risk in both species, Mitchell et al. (1991) proposed that group living in squirrel monkeys was most likely to be a response to protection from predators, in line with the model proposed by van Schaik (1989). In a similar test, Barton et al. (1996) compared two baboon species, the female-bonded Papio anubis and the non-female-bonded $P$. ursinus. In line with the model predictions (van Schaik 1989), Barton et al. (1996) found that $P$. anubis fed mostly on highly clumped food sources in contrast to $P$. ursinus habitat where such discrete foods were rare. The difference was also reflected in much higher supplant rates and stronger group cohesion among $P$. anubis compared to $P$. ursinus. While neither species was under strong between-group contest competition, predation pressure was found to be high only for $P$. anubis. Barton et al. (1996) proposed that low predation risk would allow individuals to spread out more, thus relaxing within-group contest competition even in species feeding on contestable foods. Koenig et al. (1998) compared populations of Hanuman langurs (Presbytis entellus) differing in habitat and 
foraging strategies. The provisioned populations seem to be characterized by a mix of withingroup contest and scramble competition because females are philopatric and dominance rank is positively correlated with fertility, yet female fertility has an inverse correlation with group size. In contrast, the forest population has access to superabundant, continuous food patches, and females have weakly developed dominance relationships including relatively frequent dispersal. The comparison by Koenig et al. (1998) demonstrated that the principles of the socio-ecological model also explain differences between populations of the same species.

However, the increasing number of tests have also drawn attention to the mismatches between predictions and observed behaviours, and exposed some of the limitations of the model (reviewed by Clutton-Brock and Janson 2012, Koenig et al. 2013), culminating in a call of disposing the synthetic model altogether (Thierry 2008). As an alternative, Thierry (2007) has proposed that the variation in social systems may derive from correlated trait evolution due to genetic mechanisms such as pleiotropy i.e. a single locus of a gene affects two or more phenotypic traits (Stearns 2010). This relates to another point of criticism concerning the model, namely that phylogeny may explain more of the variation than ecological variables because traits may persist due to phylogenetic inertia even in the face of changing ecological conditions (Thierry 2008). As a counter argument, it has been noted that discerning patterns of phylogenetic constraints post-hoc fails to contribute towards our understanding of what processes initially shaped these patterns (Koenig and Borries 2009). Studies of hybrid species might help to illustrate, how genetic predispositions shape social behaviour (Kappeler and van Schaik 2002). Phylogenetic inertia can also arise due to niche conservatism and the socio-ecological model does test for this effect (Koenig and Borries 2009). Another point of criticism has concerned the models' strong emphasis on competition while overlooking the importance of cooperation and affiliation (Sussmann et al. 2005). From a broader taxonomic point of view, the field of primate-socio-ecology has received general critique for not paying enough attention to the socio-ecology of non-primates (Hauser 1993, Harcourt 1998). However, it may not always be meaningful to address specific hypothesis on primates, given the large qualitative differences in life history traits across different taxa (Kappeler and van Schaik 2002).

The synthetic socio-ecological model is admittedly complex, rendering sufficient tests of its assumptions and predictions difficult (Clutton-Brock and Janson 2012). Future revisions of the model might well lead to increased complexity in case additional factors, such as 
parasites, thermoregulation, paternal relatedness among females or male strategies, can increase its explanatory power (Janson 2000). As model complexity increases, shifting from a verbal model to mathematical modeling might be a reasonable alternative to improve the interpretability of multi-causal relationships (Dunbar 2002, Koenig and Borries 2009). Despite some of the shortcomings of the synthetic model, it retains considerable explanatory power concerning key aspects such as the form of competition, making a strong case against abandoning the whole model (Koenig and Borries 2009, Clutton-Brock and Janson 2012). It has been argued that several of the discrepancies between the model predictions and observed behaviours have resulted from either incomplete tests based on limited information, or uncritical testing based on assumed relationships between causes and effects (Koenig and Borries 2009, Schülke and Ostner 2012). One of the main avenues for improving further tests of the socio-ecological model is to obtain better quantitative measures of the relevant parameters (Koenig 2002, Isbell and Young 2002). However, measuring rates of predation or infanticide have been challenging because both are relatively uncommon occurrences (Janson 2000). Predation rates tend to overlook the impact of countermeasures against predation, and a similar point has been made concerning infanticide rates (Kappeler and van Schaik 2002). One possible way to overcome this issue is to distinguish between intrinsic predation risk and the observed predation rate to gain a clearer picture of population responses predators, with a similar approach applying to understanding female counterstrategies to infanticide (Hill and Lee 1998, Janson 2000, Kappeler and van Schaik 2002). In comparison, there has been considerable progress in improving quantitative measures of food resource characteristics, another key parameter of the model (Isbell et al. 1998, Pruetz and Isbell, 2000, Koenig and Borries 2006, Vogel and Janson 2007). Spurious results of earlier tests may have resulted from reliance on broad dietary categories (e.g. folivory vs insectivory vs frugivory) in reflecting food distribution, size and quality (Koenig 2002, Snaith and Chapman 2005). The categorical approach can mask temporal and spatial variation in food quality for example when leaves in different phytophases contain different amounts of nutrients (Koenig et al. 1998, Koenig 2000).

A related issue is that quantitative measures of food resource characteristics are very sensitive to scale (Wiens 1976, Isbell and Young 2002). Terms used in the verbal model for describing foods, such as patchy or clumped, have led to some confusion in terms of the scale they refer to. There is strong agreement however, that the different characteristics of foods, including quality, patch size and dispersion, have to be measured on the scale of the consumers i.e. based on an area over which the group spreads during feeding (Schülke and 
Ostner 2012). Measures on group spread, repeated use of food patches and distances between food patches of similar quality should also be included in studies examining the proximate mechanisms influencing contest competition (Koenig 2000). Food resource distribution and abundance have often been measured from a botanical perspective, which may not reflect a relevant scale for the feeding animals (Vogel and Janson 2007).

An important methodological development, the focal tree method (Vogel 2005, Vogel and Janson 2007, Vogel and Janson 2011) incorporates measures of patch size in relation to the feeding group, the amount, quality and depletion rate of foods available in the patch, and availability of alternative foods in the near vicinity of the patch. The focal tree method also incorporates the measurement of food intake rates of all individuals feeding in the patch simultaneously, and the occurrence of agonistic interactions (Vogel 2005). These measures are important parameters for assessing net energy gain, because they link to the proximate mechanisms of the different forms of competition. Quantifying food characteristics or behavioural mechanisms, such as frequency of aggression, alone are not sufficient to test the model predictions rigorously. A study by Koenig et al. (2013) investigated the link between rates of agonism and the degree of despotism of dominance hierarchies in female primates, and they did not find support for the model prediction, that higher rates of agonism were associated with despotism, as quantified via the directional consistency index. The frequency of aggression may not always reflect the full picture of within-group contest competition (Koenig 2002). In line with game-theoretical prediction for match conflict partners, low-ranked individuals may avoid high-ranked individuals and this can still lead to dominance rankrelated skew in energy intake (Janson and van Schaik 1988, Wright et al. 2014). Avoidance is just one of the possible ways to mitigate the effects of feeding competition, as cheek-pouch use (in cercopithecoids) and fission-fusion dynamics are thought to have evolved as counterstrategies to feeding competition and hence should be accounted for when testing the model (Smith et al. 2008, Ostner and Schülke 2012).

The socio-ecological model takes an evolutionary perspective of fitness maximization and therefore it is necessary to obtain measures of the ultimate fitness effects via measures of net energy gain or reproductive success (Koenig and Borries 2009). Another major point of the socio-ecological model is the association between strong within-group contest and female philopatry, which has received perhaps most of the criticism (Clutton-Brock and Janson 2012). This prediction relies on inclusive fitness theory whereby individual traits, such as giving costly support during agonistic interactions, not only impact the individuals own 
fitness, but also the fitness of its genetically related social partners (Gardner et al. 2011). In contrast, remaining with relatives can also increase local competition between relatives, which may end up reducing the selective advantages of supporting kin (West et al. 2002). A possible alternative explanation is that the contrasts in female philopatry derive from male life-histories, whereby females disperse to avoid the risks of inbreeding with male relatives (Clutton-Brock and Janson 2012).

In sum, very few studies have comprehensibly tested most of links from resource characteristics to competitive regimes to energetic effects, female social relationships, dominance hierarchies and dispersal patterns. Given the innovations in the development of non-invasive biomarkers for energy balance, and in consumer-oriented methods for measuring food characteristics, the opportunity is better than before for improved and rigorous tests of the socio-ecological model and advancing our understanding of how ecology influences social behaviour.

\section{Study species}

Bonobos are an exemplary species for studying the adaptive significance of female gregariousness and for gaining an insight of the evolution of social tolerance and socio-sexual behaviours. Bonobos, together with the closely related chimpanzees, are the closest extant relatives of humans. Thus, understanding the selective pressures on female social behaviours in bonobos may also yield important insights in to the evolution of human social behaviours. For my thesis, I collected data on wild female bonobos at the field site of Luikotale Bonobo Research Project, co-managed by Dr. Gottfried Hohmann (Max Planck Institute for Evolutionary Anthropology, Germany) and Dr. Barbara Fruth (Liverpool John Moores University, U.K.). The field site was established in 2002 and behavioural data has been collected on the habituated Bompusa community (37-40 individuals including immatures) continuously since 2007 .

The two Pan species have been estimated to have split approximately two million years ago (Pruefer et al. 2012). However, there is indication of gene flow from bonobos into chimpanzees around 200,000-550,000 years ago (de Manuel et al. 2016). The time when the Pan clade and humans shared the most recent common ancestor has been estimated to 7 to 12 million years ago (Langergaber et al. 2012). As a species endemic to the Democratic Republic of Congo, bonobos have been geographically isolated from chimpanzees and gorillas for a considerable time due to a barrier formed by the vast Congo river (Takemoto et 
al. 2015). In contrast, chimpanzee subspecies (P. t. verus, P.t. schweinfurthii, P.t. troglodytes, $P$. t. ellioti) occur over a wider geographical range across Africa, also in sympatry with gorillas (Prado-Martinez et al. 2013). The bonobo habitat is dominated by mixed tropical forest often containing swampy areas and savannah mosaic patches (Beaune et al. 2013b, Gruber and Clay 2016).

Bonobos, known as pygmy chimpanzees in initial descriptions (Susman 1984), live in multi-male multi-female social groups called communities (Furuichi 2011). The majority of female bonobos disperse from natal communities at the time of puberty, and hence their dispersal pattern contrasts that of many Old World monkeys with male dispersal and female philopatry (Eriksson et al. 2006, Gruber and Clay 2016). Most of the female-female dyads in a bonobo community are not closely related (Gerloff et al. 1999), despite the exceptions where females remain in natal communities (Hashimoto et al. 1996, Moscovice et al. 2017). Bonobo communities are characterized by a high degree of fission-fusion dynamics, where individuals split to form smaller, temporary subgroups called parties (Kuroda 1979, White 1996). Parties can vary in size, composition and temporal duration. Bonobos feed on both plant and animal matter but they primarily consume a frugivorous diet (Hohmann et al. 2010). Bonobos feed relatively often on what appears monopolisable fruits that can be quite large in size and high in nutrient content (Hohmann and Fruth 2002). Such food resources could be expected to promote within-group contest competition resulting in rank-related skew in energy balance. Bonobos also consume piths of terrestrial herbaceous vegetation, as they offer an important source of protein that is available year round and are therefore not considered a fallback food (Malenky and Stiles 1991, Malenky and Wrangham 1994, White 1998). A high degree of fission-fusion dynamics in both Pan species has been suggested to be an adaption to their feeding ecology, as both species rely on food resources that fluctuate spatially and temporally (Hohmann et al. 2010). Chimpanzees also share several other characteristics with bonobos, including similar morphology, multi-male multi-female community structure with female dispersal, and strong adherence to frugivory (Jungers and Susman 1984, Morin et al. 1994, Hohmann et al. 2010).

Bonobos differ in a number of important traits however, particularly in terms of female sociality and aspects of socio-sexuality (White 1989, Chapman et al. 1994, Gruber and Clay 2016). Female bonobos are more gregarious compared to female chimpanzees (Furuichi 1989, Hohmann and Fruth 2002), albeit some chimpanzee communities also show relatively high female gregariousness (Wittiger and Boesch 2013, Wakefield 2013). Female bonobos 
engage in socio-sexual behaviour referred to as genito-genital rubbing (Kuroda 1980). Genito-genital rubbing is proposed to have evolved to mitigate conflicts, facilitate gregariousness, and coalition formation (Kano 1980, Hohmann and Fruth 2000, Clay and de Waal 2014). Moreover, the maximally tumescent swelling phases of female bonobos last longer than compared to female chimpanzees (Kano 1982, Deschner et al. 2003, Douglas et al. 2016).

Relationships between neighbouring communities of bonobos tend to be more peaceful than in chimpanzees (Fruth and Hohmann 2018, Sakamaki et al. 2018). Overt aggression between female bonobos is low and agonistic coalitions are more prevalent among females than among males (Furuichi 1997, Surbeck and Hohmann 2013, Tokuyama and Furuichi 2016). Female bonobos are codominant with males, such that some females are higher-ranking than males yet high-ranked males dominate several lower-ranking females (Surbeck and Hohmann 2013). Dominance hierarchies among females tend to be non-linear, and the older females seem to occupy higher ranks (Vervaecke et al. 2000, Tokuyama et al. 2017). Female ranks are relatively challenging to infer due to the lack of interactions between some female-dyads, and a cluster of females tends to share a high dominance rank in the mixed-sex hierarchy (Surbeck et al. 2012). Bonobos exhibit more tolerance compared to chimpanzees during experimentally set co-feeding situations (Parish 1994, Hare et al 2007, but see Cronin et al. 2015). However, during an experimental food transfer situation, bonobos have been shown to share less and non-reciprcally in contrast to chimpanzees, which shared more actively and reciprocally (Jaeggi et al 2010).

The diet of bonobos is proposed to differ from chimpanzee diet in two prominent aspects; bonobos regularly consume herbaceous foods (Lomako, Badrian and Malenky 1984; Wamba, Kano and Mulawwa 1984; Yalosidi, Kano 1983), and bonobo habitats seem to contain more diverse fruit sources (Malenky et al 1994). The Resource Abundance hypothesis is an amalgam of sub-hypotheses proposing that higher gregariousness of female bonobos compared to female chimpanzees is driven by food resource characteristics. The first sub-hypothesis by Wrangham (1986) proposes that feeding competition in bonobos is relaxed because they regularly consume terrestrial herbaceous vegetation occurring in evenly distributed, large patches. In addition, bonobos do not occur in sympatry with gorillas, which also feed on terrestrial herbaceous vegetation. According to Wrangham (1986), female bonobos afford higher gregariousness than female chimpanzees due to the proximate mechanism of higher terrestrial herb availability, and female gregariousness is ultimately 
favoured because female bonobos need male protectors against intraspecific harassment and thus require tolerance to travel with other females. Malenky and Stiles (1991) found that despite widely distributed terrestrial herbs at Lomako, bonobos showed selectivity for the species (Haumania liebrechtsiana) and preference for herb patches containing larger stems, which did not support Wrangham's (1986) hypothesis. Malenky and Wrangham (1994) assessed terrestrial herbaceous vegetation consumption at Lomako further by analysing faecal samples from bonobos at Lomako and chimpanzees at Kanyawa, Uganda. In support of Wrangham's (1986) hypothesis, terrestrial herbaceous vegetation was more abundant at Lomako and bonobos consumed more of it compared to chimpanzees. Malenky and Wrangham (1994) noted that chimpanzees at Kanyawara kept searching for scarce fruits even when terrestrial herbaceous vegetation was available, with the implication that terrestrial herbs could not ameliorate feeding competition for chimpanzees, as it seemed to do for bonobos.

A follow-up article by Wrangham et al. (1996) proposed an adjustment to his original 1986 hypothesis, which steered the focus from the superabundance of terrestrial herbaceous vegetation toward its quality and abundance. The new hypothesis, presented in Wrangham (2000), proposed that bonobos were able to feed on high-quality terrestrial herbaceous vegetation between fruit patches (feed-as-you-go-foraging), unlike chimpanzees. In contrast, chimpanzee mothers preferred smaller parties because of their higher costs of travelling compared to males (or females without dependent infants) in the absence of feed-as-you-go opportunity.

Another sub-hypothesis of the Resource Abundance hypothesis proposes that the greater female gregariousness in bonobos is facilitated by access to larger fruit tree patches compared to the chimpanzee habitat (Wrangham and White 1988). By comparing data on party size and fruit tree patch use by bonobos at Lomako with those used by chimpanzee at Gombe, the authors suggested that on the ultimate level, female bonobos might benefit from greater sociality through the defense of large food patches from other females. This suggestion is further discussed by White and Lanjouw (1992), and by Wrangham (1993), who shifted his views by agreeing that the benefit of sociality in female bonobos was femalefemale alliances against males, rather than male protection of females. A comparison between average party size and fruit tree patch sizes between bonobos at Lomako and chimpanzees at Kanyawara, Uganda, by Chapman et al. (1994) did not find support for the 
proposition that larger average party sizes in bonobos were due to access to larger fruit tree patches and indicated that fruit tree patch sizes vary site-specifically.

The Resource Abundance hypothesis also contains a sub-hypothesis by Malenky (1990), which proposes that there is less seasonal variation in fruit production in bonobo habitats compared to chimpanzee habitats. Data on fruit abundance and dietary breath from Lomako showed that despite some variation in fruit abundance, there did not seem to be periods of fruit scarcity that would have prevented female bonobos gregariousness, in support of Malenky's hypothesis (White 1998). In contrast, Wrangham et al. (1996) has argued that fruiting seasonality would not be expected to differ consistently between the habitats of the two Pan species because of the strong link between seasonality and latitude. Taken together, the Resource Abundance hypothesis posits that the costs of scramble competition are lower to bonobos due to their habitat characteristics, compared to most populations of chimpanzees. The hypothesis predicts weakly developed female dominance relationships, and similar energy balance and glucocorticoid levels among female community members. Instead of rank-dependent variation, energy balance and glucocorticoid levels are expected to vary seasonally with food abundance.

An alternative hypothesis, the Cooperative Defense hypothesis (Parish 1996, Vervaecke et al. 2000) proposes that bonobos are under strong within-group contest competition for limiting food resources, and that increasing female aggregation facilitates the likelihood of female-female coalitions. The benefit of female-female coalitions derives from the improved ability to defend access to food resources against males. In dyadic conflicts, males can be dominant over females and hence the additional benefit would be protection against sexual coercion by males (Parish 1996). Therefore, it offers an ultimate level explanation for the higher female gregariousness in bonobos rather than addressing how it (gregariousness) is achieved. The hypothesis puts more emphasis on defendable food resources, as it was in part inspired by behavioural observation of captive females of both Pan species provided with a defensible food resource. Under these circumstances, the female bonobos monopolised access to the resource with high rates of co-feeding, and had higher feeding success than the male bonobo, whereas in the group of chimpanzees the male and females had similar feeding success (Parish 1994). Dominant female bonobos have been found to tolerate subordinate females during co-feeding in captivity (Vervaecke et al. 2000), in line with the finding that socio-sexual behaviour of female bonobos increases with increasing potential contest competition (Hohmann and Fruth 2000, Hohmann et al. 2009). 
According to the predictions of the Cooperative Defense hypothesis, within-group contest competition for food resources leads to dominance rank effects on female net energy intake. However, because all females benefit from coalition formation against males, high-ranked females are expected to tolerate low-ranking females in exchange for their coalitionary support. Although high-ranking females are expected to be in better energy balance, the leverage by low-ranked females on high-ranked females (i.e. through the enforced tolerance) is expected to show as increased glucocorticoid levels in high-ranked females due to social stressors. In sum, the hypothesis predicts that coalitions should mainly form between females (to target males), better energy balance with increasing dominance rank and increasing glucocorticoid levels with increasing dominance rank.

Finally, the Priority of Access hypothesis offers another ultimate-level explanation. It bases itself on females forming coalitions against other females to exclude them from food resources, a pattern commonly observed in cercopithecines (Wrangham 1980, Strier 1994, Cords 2012). The Priority of Access hypothesis predicts that within-group contest competition for limiting food resources leads to female-female coalitions against other such coalitions, albeit the possibility that females sometimes ally with males is not excluded. Females are predicted to maintain close affiliative and supportive relationships with their coalition partners, which may provide an advantage against other alliances and leads to increase net energy intake for its members. Kin support is not expected to lead to the formation of a linear dominance hierarchy, because most females disperse from their natal community. Instead, the dominance hierarchy is expected to be stratified, with the strata corresponding to various female-female coalitions. In sum, females are expected to form coalitions against other females, higher-ranked females are expected to be in between energy balance, and glucocorticoid levels are expected to increase with decreasing cluster rank such that females sharing the lowest rank strata have the highest glucocorticoid levels.

\section{Thesis aims}

My study aims to clarify the adaptive significance of female-female social relationships in a multi-male multi-female primate group characterized by female natal dispersal, with implications for the emergence of cooperation and social tolerance in pre-industrial human societies. By using an approach that combines behavioral data, feeding data from the perspective of the bonobos, and hormonal data on female energy balance and allostatic load, I will examine the link between repeated social interactions in food patches and their ultimate 
outcome for proxies of female fitness. As a corollary, my study aims to elucidate which factors drive the variation in female sociality within the Genus Pan by testing between three socioecological hypotheses proposed to explain not only the higher female gregariousness in bonobos but also their enhanced tolerance. In the following paragraphs, I provide summaries for the specific goals of my two chapters.

Chapter 1 focuses on assessing the relative importance of feeding competition among female bonobos. My goal is to obtain quantitative measures to properly test the predictions of the socio-ecology model. Bonobos are classified as dispersal-egalitarian according to the socio-ecological framework (Table 2; Sterck et al. 1997). Under this classification, contest competition is not expected to be important (neither within nor between groups), female bonobos are not expected to form linear dominance hierarchies, and dominance rank is not expected to affect net energy gain. A test for contest competition effects in bonobos is particularly pertinent, given that both the fission-fusion dynamics and the genito-genital rubbing may already serve as counterstrategies for mitigating feeding competition among females. To obtain the appropriate measures, I employ the focal tree method to collect data on food intake rates of all co-feeding individuals in the patch, along with data on food resource characteristics from a perspective relevant to the feeding bonobos. In addition, I collect data on agonistic interactions, individual movements in food patches, and size and composition of feeding groups. In combination with hormonal data on C-peptide levels, I will be able to address on an unprecedented temporal resolution, whether food characteristics lead to dominance rank effects in female energy on a day-to-day basis. My additional goal is to evaluate three alternative hypotheses (Resource Abundance, Cooperative Defense, Priority of Access) on the evolution of the social system of bonobos.

In Chapter 2, I use my findings concerning female energy balance to examine ecological and social effects on female glucocorticoid levels to gain a broader understanding of the costs of sociality. To this end, I assess the relationship between female reproductive state, energy balance and dominance rank on the glucocorticoid metabolite levels as a proxy of female allostatic load. Using the allostatic load framework, my goal is to disentangle the relative effects of nutritional and social stressors. According to the allostatic load theory, highranked female bonobos are predicted to have elevated glucocorticoid levels due to the costs of acquiring and maintaining their position in the dominance hierarchy without the support of female relatives. At the same time, the glucocorticoid levels of low-ranked females are 
expected to be reduced due to coping mechanisms, including the flexibility offered by fissionfusion dynamics.

Finally, I merge the summary results of my two studies in the General discussion. I discuss the implications and significance of my findings in the broader framework of mammalian socio-ecology and conclude with an outlook and suggestions for future research. 


\title{
CHAPTER 1
}

\section{The "tolerant chimpanzee" - towards the}

\section{costs and benefits of sociality in female bonobos}

\author{
Niina O. Nurmi ${ }^{1,2}$, Gottfried Hohmann², Lucas G. Goldstone², Tobias
} Deschner $^{2}$ and Oliver Schülke ${ }^{1,4}$

${ }^{1}$ Department of Behavioral Ecology, JFB Institute for Zoology/Anthropology, University of Göttingen, Germany, ${ }^{2}$ Department of Primatology, Max Planck Institute for Evolutionary Anthropology, Germany, ${ }^{3}$ Graduate School of Systemic Neurosciences, Ludwig Maximilians University, Germany, ${ }^{4}$ Research Group Social Evolution in Primates, German Primate Center, Leibniz Institute for Primate Research, Göttingen, Germany.

Behavioral Ecology 2018; 29(6): 1325-1339 


\section{ABSTRACT}

Humans share an extraordinary degree of sociality with other primates, calling for comparative work into the evolutionary drivers of the variation in social engagement observed between species. Of particular interest is the contrast between the chimpanzee (Pan troglodytes) and bonobo (Pan paniscus), the latter exhibiting increased female gregariousness, more tolerant relationships, and elaborate behavioral adaptations for conflict resolution. Here we test predictions from three socio-ecological hypotheses regarding the evolution of these traits using data on wild bonobos at LuiKotale, Democratic Republic of Congo. Focusing on the behavior of co-feeding females and controlling for variation in characteristics of the feeding patch, food intake rate moderately increased while feeding effort decreased with female dominance rank, indicating that females engaged in competitive exclusion from high quality food resources. However, these rank effects did not translate into variation in energy balance, as measured from urinary C-peptide levels. Instead, energy balance varied independent of female rank with the proportion of fruit in the diet. Together with the observation that females join forces in conflicts with males, our results support the hypothesis that predicts that females trade off feeding opportunities for safety against male aggression. The key to a full understanding of variation in social structure may be an integrated view of cooperation and competition over access to the key resources food and mates, both within and between the sexes.

\section{KEYWORDS}

feeding competition, social foraging, C-peptide, energy balance, Pan paniscus 


\section{INTRODUCTION}

Complex sociality is a hallmark feature of the human condition with evolutionary roots in our primate heritage (Brown et al. 2011, Freeberg et al. 2012, Bergman and Beehner 2015). Our closest living relatives, bonobo (Pan paniscus) and chimpanzee (Pan troglodytes) diverged approximately 1.5-2.6 million years ago, long after the Homo-Pan split occurred 7-13 million years ago (Langergraber et al. 2012, de Manuel et al. 2016). Nevertheless, the two Pan species differ in several important aspects of their sociality. Understanding the drivers of these differences and the factors underlying bonobo sociality may inform us about the evolutionary pressures leading to characteristic traits of human sociality.

Bonobos and chimpanzees live in relatively large multi-male, multi-female groups (Miller et al. 2014, Surbeck et al. 2017) with male philopatry and female dispersal (Morin et al. 1994, Gerloff et al. 1999, Eriksson et al. 2006), and a high degree of fission-fusion dynamics (Sugiyama 1968, Kuroda 1979, Aureli et al. 2008). Despite the considerable diversity among chimpanzee subspecies (Boesch 2009, Boesch et al. 2002, Gruber and Clay 2016), differences between bonobos and chimpanzees remain salient. Compared to chimpanzees, bonobo females are more gregarious (Hohmann et al. 1999, Hohmann and Fruth 2002), more central in the social network (Wrangham 1986, Furuichi 1989, White 1989, Tokuyama and Furuichi 2017), and travel in mixed sex subgroups more often (Wrangham 1986, White 1988, Furuichi 1989). In stark contrast to chimpanzees, female bonobos are codominant with males (Furuichi 1997, Surbeck and Hohmann 2013) and evolved elaborate socio-sexual behaviors that mitigate conflicts and facilitate gregariousness and coalition formation (Kano 1980, Hohmann and Fruth 2000, Clay and de Waal 2014), which may explain why levels of overt aggression between females are low (Furuichi 1997, Surbeck and Hohmann 2013). In contrast to chimpanzees, agonistic coalitions among bonobos are more prevalent among females than males (Stevens et al. 2006, Surbeck and Hohmann 2013, Tokuyama and Furuichi 2016).

Increased gregariousness and cooperativeness may result from higher social tolerance in bonobo compared to chimpanzee females. However, feeding experiments comparing captive groups of bonobos and chimpanzees have produced inconclusive results concerning tolerance towards conspecifics, partly because they concern both male and female behavior. Parish (1994) and Hare et al. (2007) both found bonobos to be more tolerant than chimpanzees during co-feeding experiments, whereas Cronin et al. (2015) found that bonobos exhibit less social tolerance than chimpanzees. In a food transfer- 
situation, bonobos were found to share less and unidirectionally whereas chimpanzees tended to share more actively and reciprocally (Jaeggi et al. 2010). Hare and Kwetuenda (2010) found that bonobos actively provided unrelated individuals with access to food, but Bullinger et al. (2013) were not able to replicate these results.

Rooted in theory of optimal foraging in patchy environments (Charnov 1976) and socio-ecological models (Jarman 1974), three feeding ecology hypotheses have been put forward to explain patterns of agonistic behavior in bonobos that all emphasize female competition (Stockley and Bro-Jørgenson 2011). These hypotheses borrow from ecological theory (Bradbury and Vehrencamp 1976) in predicting that characteristics of limiting food resources determine how animals compete for access to these resources, both within and between groups, and propose that the competitive regime shapes the rules of social engagement (Wrangham 1980, van Schaik 1989, Isbell 1991). If food resources occur in patches small enough to be economically monopolized against group mates and qualitatively worth defending, within-group contest competition over access to these resources ensues promoting competitive exclusion if alternative patches close by provide lower nutritional yield. Experimentally clumped resources cause increased direct competition in mammals (Monaghan and Metcalf 1985) birds (Sol et al. 1998), fish (Ward et al. 2006), and invertebrates (Cameron et al. 2007) and the resulting competitive exclusion selects for the evolution of despotism (Milinski and Parker 1991), steep linear dominance hierarchies, and coalition formation (Sterck et al. 1997). If patches are too large, of low quality, or too small to be defended economically, within-group scramble competition will prevail like in social spiders fed very small or very large prey (Sharpe and Avilés 2016), promoting an adjustment of group size or feeding party size to patch size without affecting agonistic behavior (Wrangham 1980, van Schaik 1989, Isbell 1991, Koenig 2002).

The first hypothesis, hereafter referred to as Resource Abundance hypothesis, was developed specifically to explain differences between chimpanzees and bonobos and has evolved over time, resulting in a number of verbal models supplementing and expanding the original idea as proposed by Badrian and Badrian (1984). The untested premise of this hypothesis is that bonobo food patches are too large and too narrowly spread to cause diminishing returns for the consumer over realistic residence times (Charnov 1976, Chapman 1988). Different versions of the Resource Abundance hypothesis highlight the size of fruit bearing trees or the widely available, terrestrial herbaceous vegetation (THV) occurring in large, un-defensible patches both of which are thought to prevent contest 
competition, diminish within-group scramble competition, and reduce the costs of grouping among female bonobos (Badrian and Badrian 1984, Wrangham 1986, White and Wrangham 1988). The THV sub-hypothesis suggests that contest competition effects, possibly occurring during exploitation of smaller fruit patches, can be compensated by subordinates feeding more on overabundant THV. Indeed, bonobos regularly consume THV at all major study sites (Kuroda 1979, Kano 1983, Badrian and Malenky 1984), but data concerning differences in THV consumption between bonobos and chimpanzees are inconclusive (Wrangham 1986, Malenky and Stiles 1991, Malenky and Wrangham 1994, Malenky et al. 1994) and rank effects on THV feeding have not been assessed yet. The fruit patch size sub-hypothesis invokes the size of fruit patches to be larger in bonobo habitat than chimpanzee habitat (Badrian and Badrian 1984, White and Wrangham 1988). The validity of the fruit patch size hypothesis is debated (e.g. Chapman et al. 1994, Furuichi et al. 2015) but it is implicitly used as a reference for species differences in female-female social relationships in Pan (Russon and Begun 2004, Heilbronner et al. 2008, Hare and Yamamoto 2017) and crucial elements regarding bonobo feeding behavior remain untested. From the Resource Abundance hypothesis, we predict that contest competition over highly prized defendable fruit patches prevails only when patches are small whereas in large patches, contest competition is moderate or absent (White and Wrangham 1988). Apart from behavioral measures (e.g. food intake, searching time) the patch-related difference in competition can be assessed as a dominance effect on energy intake (Table 1). However, if small patch size constrains food intake, the fission-fusion system allows subordinate females to compensate for reduced intake in small patches by feeding more often or for longer time away from high-ranking females (either in nearby fruit trees or on THV) resulting in dominance rank not being related to energy balance. Accordingly, we do not expect to find dominance rank effects on food intake, feeding efficiency or energy balance, but on time spent feeding on THV and on how often a female is missing from the main party.

The other two hypotheses aim at the proximate level of competition and explain patterns of conflict and association in bonobos from resource characteristics and the way the animals compete over food but do not explain why these differ between the Pan species. While the Resource Abundance hypothesis does not specify what females can gain from grouping, the Cooperative Defense hypothesis makes a clear prediction about the tradeoffs deriving from female bonding (Parish 1996). It assumes that bonobos face strong within-group contest competition for food, and that females benefit from forming coalitions 
with other females to defend access to food resources against males. As this implies that males are dominant over females in dyadic conflicts, the additional proposed advantage for coalition formation among females is the suppression of conditioning sexual aggression (Parish 1996). The hypothesis rests on observations of captive females of both Pan species provided with a defensible food source in which female bonobos monopolized access to the food patch, exhibited high rates of co-feeding, and had a higher feeding success than the male, whereas the male had a similar feeding success as females in the chimpanzee group (Parish 1994). While these data provide convincing evidence concerning the benefits derived from female bonding, the causes for species-differences in female bonding remain ambiguous. One possible explanation is that the driving force for female bonding in bonobos is that social bonds prevent male harassment and coercion in bonobos but not in chimpanzees (Wrangham 1993). Captive female bonobos tolerate subordinate ones during co-feeding (Vervaecke et al. 2000), and intersexual dominance relations may change with partner availability (Vervaecke et al. 1999). The Cooperative Defense hypothesis predicts that contest competition over access to limited food yields dominance rank effects on energy balance, but that the effect is weak, because females exhibit tolerance towards other females in exchange for their cooperation against males. Coalitions should mainly be formed by females and should mainly target males. This is in line with data showing that it is primarily the rate of socio-sexual behavior between female bonobos that increases at times when the potential for contest competition is high (Hohmann and Fruth 2000, Hohmann et al. 2009).

The Priority of Access hypothesis derives from the common cercopithecine pattern of females forming coalitions against other females to exclude them from food resources (Wrangham 1980, Strier 1994, Cords 2012). The hypothesis assumes strong within-group contest competition for limiting food resources and female competition to be primarily intrasexual. By forming close affiliative and supportive relationships, female alliances are thought to gain an advantage against other individuals and alliances, yielding increased net energy intake to its members. Given that most females disperse from their natal group, kin support will not generate linear hierarchies to begin with. Instead, hierarchies will be stratified, with females engaging in conflict only with females from other strata but not from their own (such as the bonobo hierarchy constructed with the ADAGIO method following Douglas et al. 2017). Like the Cooperative Defense hypothesis, the Priority of Access hypothesis predicts that bonobos face strong contest competition over access to food. In contrast to the other hypotheses, the Priority of Access hypothesis predicts conflicts over 
food to occur equally often between females and between the sexes, females to form coalitions mainly against other females, and strong rank effects on food intake and feeding effort. Furthermore, the resulting energy balances would be expected to vary according to rank strata, instead of diminished variation resulting from general tolerance among females.

Table 1: The three hypotheses for the evolution of bonobo social structure with predictions

\begin{tabular}{|c|c|c|c|}
\hline & $\begin{array}{c}\text { Resource } \\
\text { Abundance }\end{array}$ & $\begin{array}{c}\text { Cooperative } \\
\text { Defense }\end{array}$ & $\begin{array}{l}\text { Priority of } \\
\text { Access }\end{array}$ \\
\hline $\begin{array}{l}\text { Female coalitions } \\
\text { target }\end{array}$ & - & Males & Females \\
\hline Food patch depletion* & No & Yes & Yes \\
\hline Food intake in patch & Not related to rank & Increases with rank & $\begin{array}{c}\text { Strongly increases with } \\
\text { rank }\end{array}$ \\
\hline Movements in patch & Not related to rank & Decreases with rank & $\begin{array}{c}\text { Strongly decreases with } \\
\text { rank }\end{array}$ \\
\hline Energy balance (uCP) & Not related to rank & Increases with rank & $\begin{array}{c}\text { Strongly increases with } \\
\text { rank }\end{array}$ \\
\hline
\end{tabular}

Here we test the competing predictions (Table 1) of the three hypotheses laid out above with data on polyadic conflicts, feeding behavior in fruit patches and THV, as well as information on individual energy balance in female bonobos in their natural habitat at LuiKotale, Democratic Republic of Congo. We use the recently refined focal tree method (Vogel and Janson 2011), which combines information on the size and quality of individual food patches with data on feeding and agonistic behavior of all co-feeding consumers. We first test whether feeding in fruit patches is associated with diminishing returns resulting from patch depletion by regressing intake rate and feeding effort over residence time. Then we relate food intake rates and feeding effort within patches to female dominance rank as a function of patch size and number of competitors. These data offer a window into food patch characteristics that are relevant to the consumer, a perspective that has been applied 
to other primates (Snaith and Chapman 2005, Heesen et al. 2014) but was previously not applied to bonobos. As indicators of conflict avoidance and compensation for reduced food intake, we relate female dominance rank to average party size and to the time spent in THV patches, respectively. Finally, we assess the energetic consequences of direct and indirect forms of feeding competition with data on variation in urinary C-peptide (uCP) levels within and among individuals. The C-peptide of insulin, as shed in urine, has been established as a non-invasive biomarker for energy balance (Deschner et al. 2008, Girard-Buttoz et al. 2011) and energetic status (Sherry and Ellison 2007, Emery Thompson and Knott 2008), including in wild bonobos (Georgiev et al. 2011, Surbeck et al. 2015).

\section{METHODS}

\section{Ethics statement}

All methods applied were strictly non-invasive and non-contact. The Institut Congolaise pour la Conservation de la Nature (ICCN) granted the permission to conduct research at LuiKotale, Salonga National Park, Democratic Republic of Congo (0683/ICCN/DG/ADG/014/KV/2012). Permits for exporting the samples from the Democratic Republic of Congo were issued by the ICCN (0521/ICCN/DG/CWB/05/01/2014), while the permits for importing the samples to Germany were issued by the state ministry for social affairs and consumer protection of Saxony/Germany.

\section{(a) Study site and study subjects}

Data were collected at the LuiKotale field site near Salonga National Park, Democratic Republic of Congo, from August 2012 to April 2013 and November 2013 to August 2014. A description of the field site can be found in Hohmann and Fruth (2003). During this period, the fully habituated Bompusa West community consisted of five adult and two subadult males along with 16 adult females and three subadult females. Of the adult females, 13 were parous, of which two gave birth during the study period, and the remaining three were nulliparous. Our subjects were 14 adult females resident in the community at the onset of the study. 


\section{(b) Behavioral observations}

We followed bonobos during half day shifts ( $N=223$ days, 577 hours focal animal sampling), either from the morning nest site until noon or from noon until the evening nest site. Due to the fission-fusion dynamics of the species, most follows were at the party level. We define a party as a subgroup of the community that may vary in size and composition of individuals over time. All data were collected by two observers working as a team using notebooks and a voice-recorder, with pre-assigned methods of data collection and consistent roles throughout the field season. For baseline data, each half hour interval, one observer recorded the party composition, as well as the activity, location (ground or tree), and any food items being consumed by each individual in sight ( $N=3644$ scans). All occurrences of agonistic behavior in the party were recorded, including the context as well as the identities and roles of opponents (Altmann 1974). Both observers carried Garmin GPS devices (GPSMAP® 62 and GPSMAP $® 60 C S x$ ) for recording bonobo ranging data and marking the food patches visited during shifts.

\section{Food patch characteristics}

We collected data on bonobo feeding behavior with a modified version of the focal tree method (Vogel 2005, Vogel and Janson 2007, Vogel and Janson 2011). We included all arboreal and terrestrial feeding patches as focal trees (Chapman 1988), in order to account for instances such as a terrestrial patch where food had fallen on an area roughly equal to the tree crown above. In a few cases, we also included adjoining tree crowns of the same species as a single focal tree, when we clearly observed that the bonobos fed and moved easily among two closely connected crowns. Each focal tree was tagged physically using clearly visible plastic flagging tape with a hand-written unique identifier code (focal tree identity) to facilitate reliable recording of potential re-visits. We estimated focal tree size based on the diameter at breast height (DBH) for single trees in centimeters (Chapman et al. 1992), the DBH $(\mathrm{cm})$ of the "mother" tree from which the food had fallen, or the sum of DBHs for adjoining tree crowns. The latter practice may have over-estimated the size of multi-stem crowns but was used for $5 \%$ of focal trees only. We visually estimated focal tree food crop size on a five-point logarithmic scale (1 = 1-9 items, $2=10-99,3=100-999,4=$ 1000-9999 and 5 = 10000-99999; Janson and Chapman 1999). Focal tree observations began when the first bonobo entered the feeding patch and ended when the last individual departed the patch. One observer recorded the identities and times of entries and exits for each individual of the feeding group, which were then used in defining the "feeding bout 
duration" and "feeding party size" of each focal tree observation. When there was a clear pause in feeding such that most bonobos started resting or grooming, the time between the pause and resumption of feeding was subtracted from the total feeding bout duration. Bonobo food plants were identified with the help of local field assistants and by using reference herbaria at camp. We visited remaining unidentified focal trees at the end of the field season with a local botany expert in order to identify the remaining plant species.

\section{Food intake}

The second observer recorded feeding behaviors during five-minute focal protocols, rotating among all focal females feeding in the patch. Feeding was defined as ingesting, chewing, swallowing and short handling/processing times (10 sec), as well as short pauses (10 sec) and short movements between consecutive food items. Intake rates were calculated as the number of food items (e.g. fruits, seeds) ingested per unit of time of uninterrupted feeding behavior. For dry seeds, such as those of Scorodophloeus zenkeri, we counted the number of seed pods processed per unit time. In the case of leaves and flowers, we recorded the number of handfuls ingested.

\section{Movement}

To quantify feeding costs, movement in the focal tree that briefly interrupted feeding activity was recorded as a dichotomous variable (moved vs did not) for every one-minute interval of focal animal observation in a focal tree protocol.

\section{Dominance rank}

A novel graph based method, ADAGIO, was developed recently by Douglas et al. (2017) to construct a dominance hierarchy among female bonobos. We used an ADAGIO chart based on the overt and subtle agonistic interaction data from our observation period to rank the 14 study females into eight rank categories. The rank categories were based on an ADAGIO top-down approach such that the highest rank $r=1$ was assigned to the females at the root nodes of the graph and the direct child-nodes of these females were assigned rank $r+1$ etc. This method is particularly well suited to female bonobo dominance relationships, as it allows a quantification of nonlinear as well as linear dominance hierarchies. 


\section{(c) Urine collection}

Urine samples were collected from all focal individuals throughout the study period opportunistically for C-peptide measurement, with a focus on obtaining the first morning urine voids, as these samples better represent nocturnal fasting (Sherry and Ellison 2007). We used the underside of large Marantaceae leaves to capture urine samples $(N=230)$ or pipetted them from foliage on the ground $(N=8)$. Urine sample collection was conditional on the absence of urine from other individuals to avoid cross contamination, and on the sample not coming into contact with feces (Higham et al. 2011). All samples were frozen in liquid nitrogen on the day of collection and shipped to the laboratory on dry ice, where they were stored in $-20^{\circ} \mathrm{C}$ until analysis.

\section{(d) Hormone analyses for energy balance}

We assayed $N=238$ urine samples with matching behavioral data from the previous day for C-peptide levels at the Endocrinology laboratory of the Max Planck Institute for Evolutionary Anthropology, Leipzig, Germany. Thawed urine samples were shaken for 10 seconds (VX-2500 Multi Tube Vortexer) and then centrifuged for ten minutes at $2000 \mathrm{~g}$ (Heraeus Multifuge). We then used solid phase Enzyme Amplified Sensitivity Immunoassay kits (C-PEP-EASIA KAP0401) commercially distributed by DIAsource. The immunoassay was designed to measure C-peptide levels in human serum, and has been validated for bonobo urine (Surbeck et al. 2015). Intra-assay coefficients of variation (CV) based on the mean CVs of 4 replicates were $3.89 \%$ for low and $3.75 \%$ for high value quality controls. Inter-assay CVs were $2.66 \%$ for low and $8.85 \%$ for high value quality controls ( $N=15$ plates). To adjust for the variation in the volume and concentration of the urine, we corrected the C-peptide levels by Creatinine (Crea) levels in each sample (Bahr et al. 2000) and reported all hormone measurements as ng C-peptide/mg Crea. Samples with Crea values lower than $0.05 \mathrm{mg}$ Crea/ml were omitted from further analyses ( $N=2$ samples, $0.84 \%$ of all assayed samples). In addition, $N=1(0.42 \%)$ sample was excluded because it failed to yield detectable C-peptide levels.

In humans, plasma insulin levels increase dramatically from middle and late pregnancy (Spellacy and Goetz 1963, Spellacy et al. 1965) due to the necessity of diverting maternal glucose towards optimal fetal growth. Seven of the resident females gave birth during our field sampling seasons. Because elevated insulin levels do not reflect energy balance, we excluded 17 urine samples from pregnant females from our analyses. To do 
so, we assessed pregnancy retrospectively from observed birth events. Reproductive status of 10 of the 14 females was also monitored with pregnancy tests strips (hCG strip Artron Bioresearch Inc.) and with urinary steroid hormone analyses for another project (Douglas et al. 2016) rendering the likelihood of undetected pregnancy to be very low. The final sample size was $N=218$ with $15.6 \pm 6.8$ SD samples per female (range $=5-28$ ).

To test whether the C-peptide assay detected known patterns of C-peptide level variation, we first compared mean uCP values during the last pregnancy trimester with mean values when not pregnant for seven females with a Wilcoxon matched pairs test and found late pregnancy values to be significantly higher $\left(\mathrm{T}^{+}=28, \mathrm{~N}=7, P=0.016\right.$; Fig. 1a) which was expected from the human literature (Spellacy and Goetz 1963). Secondly, to assess whether our assay was sensitive enough to pick up day-to-day variation in feeding behavior, we compared two clearly distinct dietary regimes; days when females consumed mainly Dialium spp. and days when they were feeding mainly on the dry seeds of Caesalpinioideae trees such as Scorodophloeus zenkeri. Dialium fruits offer easily accessible pulp, are small fruits that require minimal handling time and are easy to harvest because they are clustered in tree crowns. Moreover, they contain a relatively high amount of sugar compared to the average sugar content of other fruits consumed by bonobos (Beaune et al. 2013a). Caesalpinioideae seeds are often encased in comparably hard seed pods requiring longer handling times, and are difficult to harvest because they occur widely dispersed over the tree crown and should therefore have a much smaller effect towards a positive energy balance than Dialium. We found that average individual uCP morning values following days when the bonobos fed mainly on Dialium fruits were significantly higher than morning values following days when dry seed consumption was the main diet (Wilcoxon matched pairs test $\mathrm{T}^{+}=35, \mathrm{~N}=8, P=0.016$; Fig. $1 \mathrm{~b}$ ), in line with similar results from another bonobo community in Kokolopori (Georgiev et al. 2011). 
a)

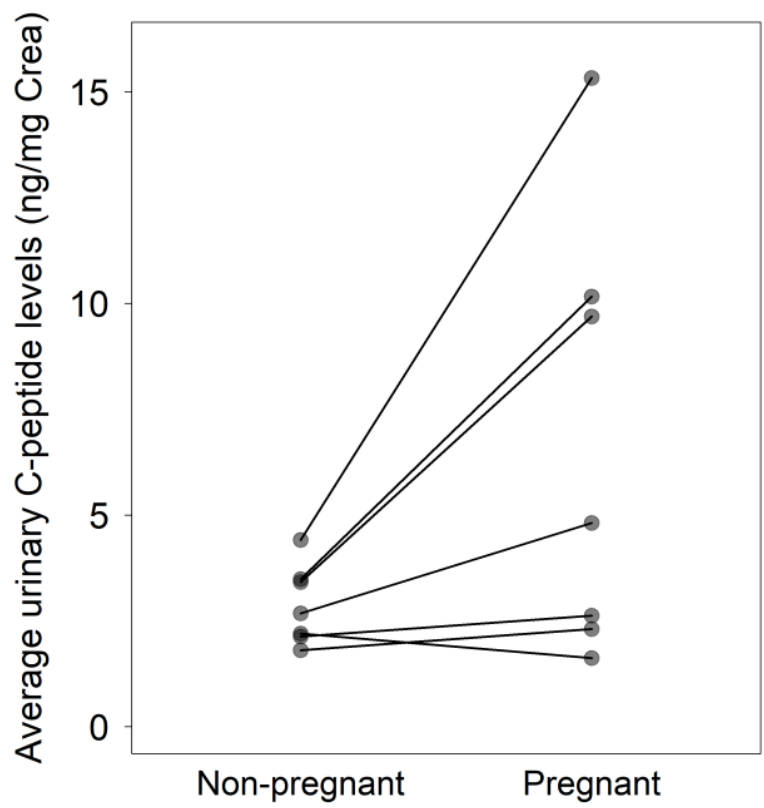

b)

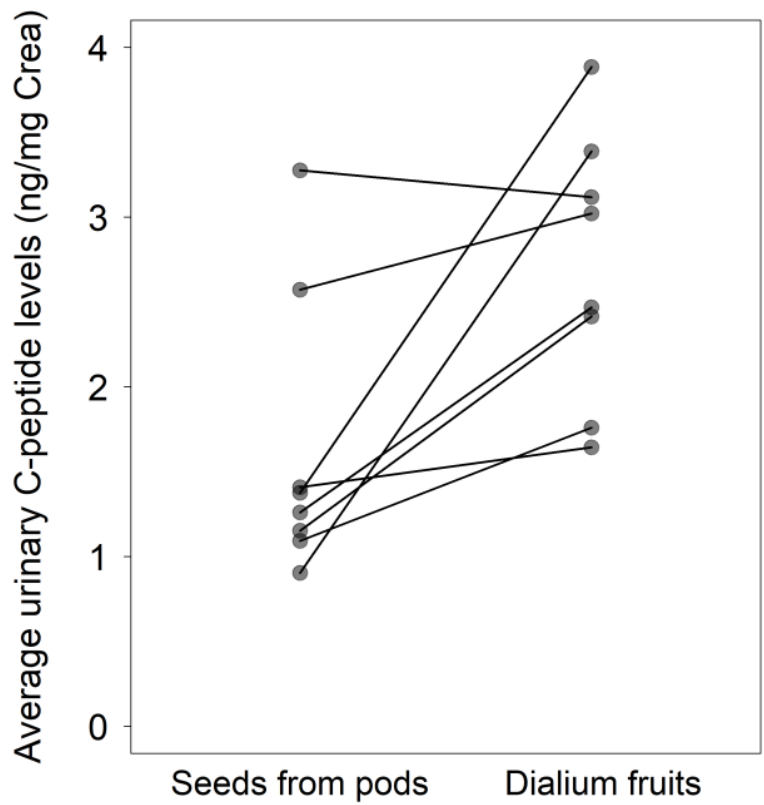

Figure 1: Average urinary C-peptide levels ( $\mathrm{ng} / \mathrm{mg}$ Creatinine) a) in nonpregnant ( $N=7$ females) and pregnant bonobo females ( $N=7$ females), and $\mathbf{b}$ ) during periods of Dialium fruit consumption and seeds of dry pods (Monopetalanthus, Hymenostegia, Scorodophleus) consumption ( $N=8$ females).

\section{(e) Statistical analyses}

We tested our predictions (Table 1) concerning the competitive regime of female bonobos with two Generalized Linear Mixed Models (GLMM; Baayen 2008) based on the behavioral data from females during focal tree observations. We then constructed a linear mixed model (LMM; Baayen 2008) to test for a potential energetic signature of competition among the females through our physiological measure, urinary C-peptide.

\section{(i) Food intake model}

In our first model, we evaluated whether social factors and food patch characteristics have an effect on female feeding gain. As an index of food intake, we used individual intake rate (bites or items/min) measured during the focal tree protocols as our response variable. We had initially built this model as an LMM with a Gaussian error structure, in which the response variable was set as the deviation of individual intake rate/min from the average intake rate for each food species, but this Gaussian model did not meet its assumptions 
about residuals, as the model residuals against fitted values were structured along diagonal lines. We then used a GLMM with a Poisson error structure with the counts of individual intakes as the response such that each minute of intake within the feeding focal protocols per female represented a single data point. We excluded incomplete intake rate minutes, unless they were preceded and followed by a minute of continuous intake. We included dominance ranks as standardized ranks per observation ranging from zero (lowest stratum) to one (highest stratum), converted from the eight rank strata derived from the ADAGIO plot and the number of individuals in the patch as our test predictors of social effects. Because the pattern of dominance among females tends to be non-linear, and nulliparous, young females are typically lower ranking than older females, we included rank as a squared term to account for a potential non-linear effect of rank on food intake resulting from only the lowest-ranking females having decreased food intake compared to mid- and high-ranking females. The number of adult and subadult individuals in the patch was determined from the continuous records of focal tree entries and exits to obtain a point score for each intake minute. After inspecting the distribution of the number of individuals, we log-transformed it to reduce the skew in its distribution.

Our test predictors accounting for food patch characteristics included the logtransformed size of the focal tree as $\mathrm{DBH}(\mathrm{cm})$ and the food crop size score. To test for the effect of food patch depletion, we included the proportion of time into the feeding bout as an additional predictor along with its squared term in case of a non-linear effect. The nonlinear effect could be expected based on the empirical observations that cumulative food intake follows a negative exponential curve due to satiation (McCleery 1977). We calculated the temporal variable by taking the difference between the time the focal tree bout ended and the time when the intake rate was recorded, and expressed it as the proportion of time into the feeding bout. We standardized the predictor to range from zero (time when the intake was recorded) to one (time when the focal tree bout ended). Due to our interest in the mode of feeding competition among female bonobos, we wanted to test whether dominance rank, our main test predictor, would have a different effect on intake rate depending on patch size, varying level of crowding or food availability. Thus, we added the following three interaction terms in to our model of food intake: i) rank squared (and rank) with the number of individuals in the patch, ii) rank squared (and rank) with $\mathrm{DBH}$, iii) rank squared (and rank) with food crop size score. Time elapsed since midnight and its square were added as control predictors because time of day has been shown to have an influence on primate feeding behavior (Carlson et al. 2013). Feeding bout identity (date combined 
with a running number for $n$th focal tree of the shift), focal tree identity, focal tree species and bonobo identity were added as random effects.

\section{(ii) Movement model}

Our second model examined the influence of the same social factors and food patch characteristics on female feeding costs. As an index of feeding cost, we tested the movement of females within focal tree food patches as a response variable in a GLMM with a binomial error structure. Each data point was one of either binary outcome "yes" or "no", corresponding to whether an individual female moved during a one-minute feeding focal recorded during the focal tree observations. We included the same test predictors as in the intake model, with the two following differences: i) we did not include a quadratic dominance rank term as we did not expect a non-linear effect of rank on movement, and ii) we included the group patch occupancy time as minutes until end of feeding bout, instead of using the proportion in to bout. Our interaction terms and random effects were the same as in the food intake model.

\section{(iii) Energy balance model}

As a final test to evaluate the mode of feeding competition among female bonobos, we used an LMM with a Gaussian error structure with the log-transformed urinary C-peptide levels, a proxy of energy balance, as the response variable. In line with our two models of behavior, we included dominance rank as our main test predictor. As an additional social test predictor, we used mean party size from the day preceding urine sample collection. Mean party sizes were calculated from the half-hourly party scan data, with the condition that the female (sampled the following morning for urinary C-peptide level) was observed in over 60 $\%$ of scans, as party size and composition can fluctuate throughout the day due to the fission-fusion dynamics of bonobo communities. We included the proportion of party scans the party spent feeding and the number of focal tree food patches visited per hour by the party as test predictors accounting for energy intake of the previous day. To calculate the proportion of feeding scans, we divided the number of half-hour party scans where most individuals of the party were observed feeding by the total number of scans recorded during the respective shift. The number of patches visited per hour variable was log-transformed due to its skewed distribution. 
Because terrestrial herbaceous vegetation availability has been proposed to relax feeding competition among female bonobos (Wrangham 1986), we included the proportion of party scans the party spent feeding on terrestrial herbaceous vegetation during the prior day as an additional test predictor. We calculated the proportion from the half-hour party scans by dividing the number of scans where most individuals of the party were observed feeding on terrestrial herbaceous vegetation by the total number of scans recorded during the respective shift, and square-root transformed the variable to make the its distribution more symmetrical.

To examine potential effects of energy expenditure on urinary C-peptide levels, we included distance travelled by the party in meters per hour of observation as a test predictor. Calculation of this parameter was based on the GPS track log of the day prior to urine sample collection; each track was first cleaned to avoid artificial increases in track length due to GPS points recorded while the party was stationary in a feeding patch. The cleaned track distance was then divided by the total duration of track recording to obtain the mean speed.

While the bonobo habitat exhibits some degree of seasonality in terms of predictable dry seasons (Beaune et al. 2013b) and there may be periods that are nutritionally better in terms of food availability, we were not able to test for effects of seasonality based on phenology data. We therefore followed Emery Thompson et al. (2014) and modeled as a test predictor the variation in food abundance as the proportion of fleshy fruit in the feeding time budget measured with 30 min group scans.

Recent work on bonobos at LuiKotale by Surbeck et al. (2015) showed that urinary C-peptide levels in male bonobos varied with dominance rank depending on the party size of the previous day, with higher-ranking males showing greater increases in urinary Cpeptide levels with increasing party size. We therefore included a two-way interaction term between rank and party size to account for the possibility that female dominance rank affects energy balance differently depending on the size of the party. We tested for two additional two-way interactions; one between dominance rank and duration of feeding and another between dominance rank and number of patches visited.

As uCP levels have been shown to be lower during the first six months of lactation in wild chimpanzees, (Emery Thompson et al. 2012) we included a dichotomous variable "lactating" vs. "non-lactating" $(N=28,12.9 \%)$ into our model as a control predictor. Bonobo 
identity and date were included as random effects to account for uneven sampling and repeated measures of individual females.

\section{iv) Model implementation}

All of our mixed models were implemented in $R$ (version 3.3.1.; R Core Team 2016) with the package "Ime4" (Bates et al. 2015) for model fitting. As a preparatory step prior to fitting the models, we normalized our numeric and ordinal input variables by z-transforming them to a mean of zero and standard deviation of one. This facilitates the interpretability of the resulting parameter estimates and squared terms (Schielzeth 2010). In addition, we included random slopes for every model between all those pairs of random and fixed effects, where one fixed effect varied within one level of a random factor in order to keep the Type I error rate at the nominal rate of 5\% (Schielzeth and Forstmeier 2009, Barr et al. 2013). For example, the same female fed in trees with different DBH's, so a random slope was modeled for the random effect of bonobo ID and the fixed effect of $\mathrm{DBH}$. The same female had always only one rank, so the random slope was not included for bonobo ID and rank.

We obtained Variance Inflation Factors (VIFs; Field 2005) determined for standard linear models (excluding the random effects, interactions, and squared terms) with the package "car" (Fox and Weisberg 2011) in order to rule out potential collinearity issues among the predictor variables. We did not find any issues with collinearity among our predictors (maximum VIF = 1.77). For the energy balance model with Gaussian error structure, we made a visual assessment of a quantile-quantile plot and the distribution of residuals plotted against fitted values to verify the assumptions of normally distributed and homoscedastic residuals, and did not detect any deviations from these assumptions. We checked model stabilities by omitting each level of random effects one at a time and comparing the derived model estimates with those of the full model estimates. The comparison of estimated coefficients did not reveal any influential cases. We checked that the food intake model with Poisson error structure complied with the model assumption of absence of overdispersion (Cameron and Trivedi 1990) and did not detect any issues with overdispersion $\left(X^{2}=2392.91, P=1\right.$, dispersion parameter $\left.=0.528\right)$.

As an initial test of significance (Forstmeier and Schielzeth 2011), we compared each of our full models against its respective null model (excluding test predictors but including random effects and respective random slopes) with a likelihood ratio test using the anova function with the test argument set to 'Chisq'. Our threshold for statistical 
significance was set to $P=0.05$. On the condition that the full model was significant against the null model, we proceeded to test for the significance of each variable one at a time (Barr et al. 2013) using the drop1 function in R. We tested the significance of interaction terms first, removing all non-significant interactions and only then testing the lower order variables that had been involved in the interactions. Our samples sizes were $N=4550$ for the food intake model, $\mathrm{N}=4500$ for the movement model, and $\mathrm{N}=218$ for the energy balance model. Details concerning the number of levels per random effect for each model can be found in Table 2. 
Table 2: Summary of models tested.

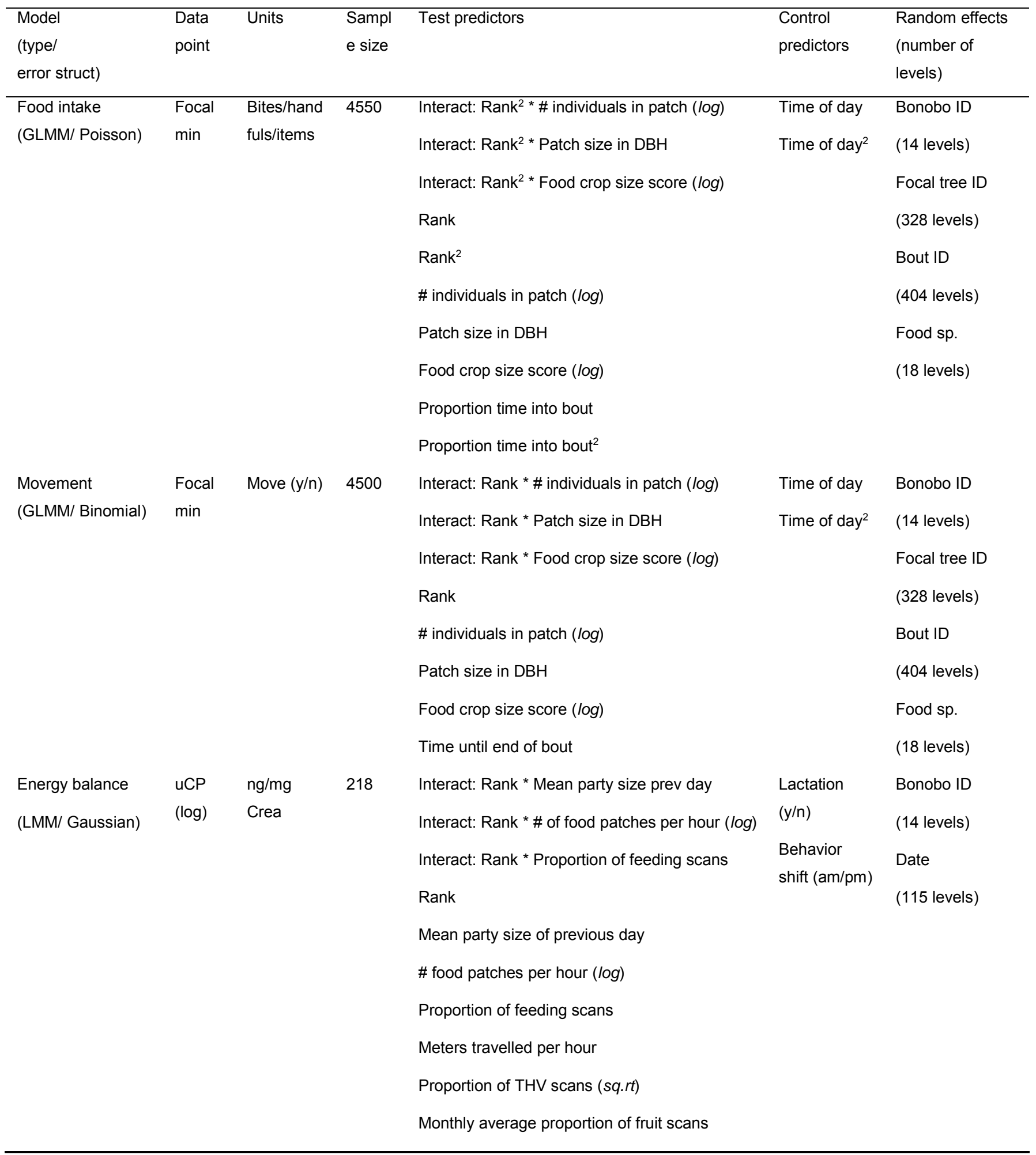




\section{RESULTS}

During the study period, females showed joint aggression against a common target 17 times (i.e. they formed a coalition sensu de Waal and Harcourt 1992) and a female supported another female in a conflict twice. The target in these conflicts was a male in 17 cases and a female in two. Considering only those conflicts that could be clearly assigned to a feeding context, 10 female-female coalitions were observed and nine targeted an adult male. Thus, females were more likely to work against males than against females in polyadic conflicts with low probabilities that these outcomes were the result of a random process (Binomial test for all conflicts $N=19, P=0.0007$, and for conflicts in feeding context $P=0.014)$. All 14 females engaged in joint aggression against male targets in a total of 17 different dyads. Comparing raw counts from all occurrences data matched per female, in dyadic conflicts in a feeding context females did not aggress males more often than females (median and interquartile range for male targets $=6(3-10)$, for female targets $=4(2-11)$, Wilcoxon matched pairs test $N=14, T=43, P=0.86)$. This pattern remained stable after exclusion of the three lowest ranking females that exhibited low or no aggression to other females in dyadic feeding conflicts $(T=26, P=0.88)$.

During the two field seasons we sampled a total of 683 focal patches, consisting of the following food types: fruits, leaves, flowers, pith, tree bark and animal matter. Most food items were fruits (611 patches; 45 taxa), including dry fruit pods (64 patches; 6 taxa) of the family Fabaceae, from which bonobos extracted seeds only (except for Scorodophloeus zenkeri). Bonobos consumed leaves (53 patches; 9 taxa), with some of the taxa appearing also in other food types, for instance, the leaves and fruit of Dialium spp. were consumed, and the leaves, flowers (6 patches) and dry fruit pods of Scorodophloeus zenkeri were consumed. The remaining focal patch observations involved the consumption of animal matter (8 patches; 4 taxa), tree bark ( 1 patch; 1 taxon) and waterlily (4 patches; 1 taxon).

Bonobos consumed Dialium in $16 \%$ (110 observations of 683) of focal trees, relatively consistently throughout both field seasons. The second most frequently used focal tree species was Polyalthia suavolensis (12\%, 80 observations), which was consumed almost exclusively during a two-month period (Nov-Dec) of the first field season, while during the second field season it was recorded only on a few days in November. Only $8 \%$ of focal tree observations concerned leaf consumption (53 observations), and 9\% were on seed feeding in Caesalpinioideae trees (64 observations). 
In order to investigate the effects of social factors and food patch characteristics on the food intake rates of female bonobos, we analyzed 404 feeding bouts that took place in 328 focal trees from 13 ripe fruit taxa, three dry fruit pod taxa, three leaf taxa, and one flower taxon. We did not include food items such as duiker meat (Philantomba monticola), jungle sop (Anonidium mannii) or African breadfruit (Treculia africana) due to the difficulty to obtain intake rates from a number of different females.

Our full model for the food intake was significant as compared to the null model (likelihood ratio test: $X^{2}=43.26$, df $=13, P<0.001$, Table 3). We found that the proportion of time until the end of a feeding bout had a significant, non-linear effect on intake rate (Table 3). Intake rates declined steeply from the beginning of a bout towards the middle and continued to decline on a shallower curve towards the termination of a bout (Fig. 2b). Together with the observation that the probability of moving in food patches increased as the minutes until the end of the bout decreased (Table 4, Fig. 3b; see the movement model below), these results suggest that the bonobos depleted their food patches.

Table 3: Food intake rate increases with increasing dominance rank and increasing food crop. The proportion of time remaining until the end of a feeding bout has a non-linear relationship with individual food intake rate. Results are an overview of a GLMM ( $N=4550$ focal minutes; mean minutes per female $\pm S D=325.0 \pm 217.7$, range $=89.0-935.0$ ), with the significant effects displayed in bold.

\begin{tabular}{|c|c|c|c|c|}
\hline & $\begin{array}{l}\text { Descr. Stats. } \\
\text { mean (range) }\end{array}$ & Estimate $\pm \mathrm{SE}$ & $z$ & $P$ \\
\hline Intercept & & $1.575 \pm 0.209$ & 7.546 & 1 \\
\hline \# individuals in patch & $5(1-17)$ & $-0.014 \pm 0.012$ & -1.210 & 0.235 \\
\hline Patch size as DBH $(\mathrm{cm})$ & $62(4-350)$ & $-0.013 \pm 0.025$ & -0.496 & 0.632 \\
\hline Food crop size score & $3.6(2-5)$ & $0.068 \pm 0.030$ & 2.245 & 0.046 \\
\hline Rank & & $0.034 \pm 0.011$ & 3.202 & 0.023 \\
\hline Proportion into bout & $0.4(0-1)$ & $-0.071 \pm 0.008$ & -8.559 & 1 \\
\hline Proportion into bout ${ }^{2}$ & & $0.028 \pm 0.010$ & 2.716 & 0.010 \\
\hline
\end{tabular}

The full model was highly significant as compared to the null model (likelihood ratio test $X^{2}=43.26, \mathrm{df}=13, P<$ $0.001)$. All interaction terms were non-significant and we therefore removed them from the model. ${ }^{1}$ Significance test not indicated because of not having a meaningful interpretation. 
The food intake model also revealed a significant positive effect of female dominance rank on intake rates (Table 3), lending support to the Cooperative Defense and the Priority of Access hypotheses but running against the Resource Abundance hypothesis (Table 1). Across all food patches, the higher ranking a female was, the more food she ingested per unit time spent in the patch, though the effect was modest (Fig 2a). The interaction between rank (including rank squared) and the number of individuals in a patch was non-significant, suggesting that the effect of rank on intake rate was similar across feeding group sizes, in support of the Cooperative Defense hypothesis. In addition, we found that the amount of food crop had a significant effect on intake rates (Table 3) such that animals feeding in focal patches with a larger crop size enjoyed higher intake rates. The observed rank effects on intake rates were not affected negatively by food crop size, as evidenced by the nonsignificance of the interaction between rank (including rank squared) and crop size, indicating that contest competition was not generally increased in patches containing less food.

a)

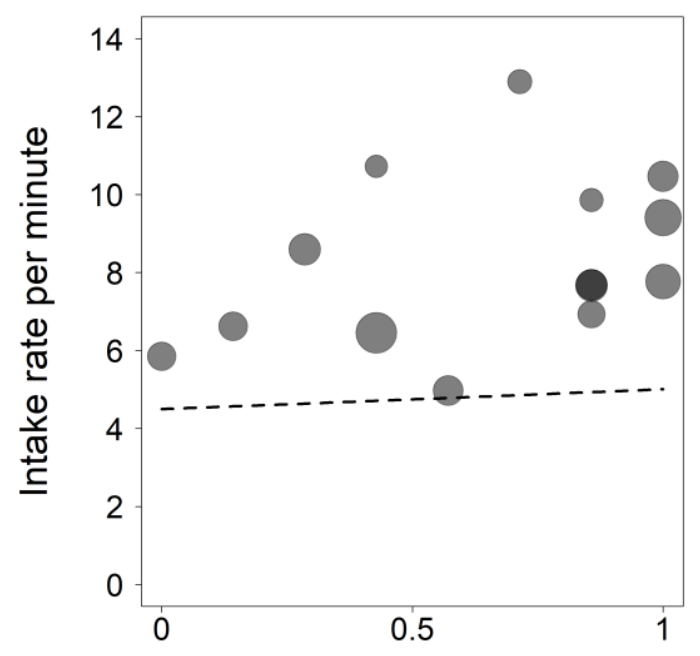

Increasing female dominance rank b)

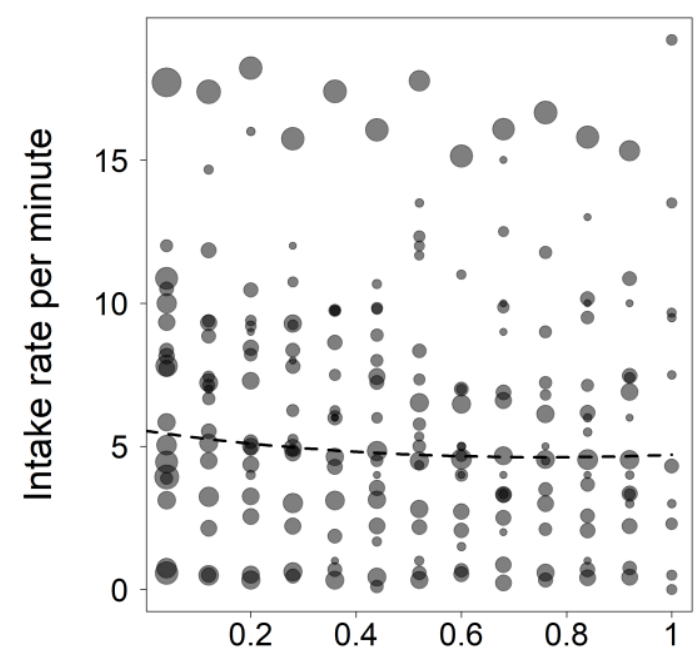

Proportion of time until end of bout

Figure 2: Food intake rate in focal trees in relation to a) female dominance rank (GLMM: estimate \pm $\left.\mathrm{SE}=0.034 \pm 0.011, \mathrm{X}^{2}=5.21, \mathrm{df}=1, P=0.023\right)$, and $\mathbf{b}$ ) the proportion of time until end of bout with 1 on the $x$-axis indicating the termination of bout (GLMM: estimate $\pm S E=0.028 \pm 0.010, x^{2}=6.73$, $\mathrm{df}=1, P=0.010$ ). The area of each circle in $a$ ) is in proportion to the number of data points per female. The area of each circle in $b$ ) is in proportion to the number of data points per food type. The dashed line in both $a$ ) and b) depicts the fitted model. 
Using the same subset of $N=404$ feeding bouts as the intake model and the same ecological and social predictors, we modeled the probability that a female moved during a minute of feeding in a focal tree which occurred in $18 \%$ of cases. Overall, our full model for movement was significant as compared to the null model (Table 4). We found a significant relationship between female dominance rank and movement, with lower ranking females being more likely to move in food patches than higher ranking females (Fig. 3a). None of the interaction terms between dominance rank and factors promoting contest competition (patch size, crop size and feeding party size) reached significance. Thus, patches did not differ much in the strength of contest competition caused. Therefore, the non-significant interaction terms were excluded from the movement model. Interestingly, we observed a statistical trend for the interaction of dominance rank and the number of co-feeding individuals to have an effect on movement probability, indicative of interference between individuals.

Table 4: The probability of movement in food patches decreases with dominance rank. The probability of movement has a negative, linear relationship with time remaining until the end of a focal tree bout, indicating that movement in focal trees increases towards the end of a feeding bout. Results are an overview of a GLMM ( $N=4500$ focal minutes), with the significant effects displayed in bold.

\begin{tabular}{lcccc}
\hline & $\begin{array}{c}\text { Descr. Stats. } \\
\text { mean (range) }\end{array}$ & Estimate \pm SE & $z$ & $P$ \\
\hline Intercept & $5(1-17)$ & $-0.102 \pm 0.104$ & -0.977 & 0.383 \\
\# individuals in patch & $62(4-350)$ & $0.088 \pm 0.133$ & 0.666 & 0.522 \\
Patch size as DBH (cm) & $3.6(2-5)$ & $0.047 \pm 0.128$ & 0.366 & 0.729 \\
Food crop size score & & $-\mathbf{0 . 1 5 0} \pm \mathbf{0 . 0 6 9}$ & $\mathbf{- 2 . 1 9 2}$ & $\mathbf{0 . 0 4 9}$ \\
Rank & $\mathbf{4 6}(\mathbf{0 - 2 4 9 )}$ & $\mathbf{- 0 . 2 6 1} \pm \mathbf{0 . 0 8 8}$ & $\mathbf{- 2 . 9 7 6}$ & $\mathbf{0 . 0 1 0}$ \\
Minutes until end of bout & & $-1.349 \pm 0.194$ & -6.948 \\
\hline The full model was highly significant as compared to the null model (likelihood ratio test $\mathbf{X}^{2}=21.43, \mathrm{df}=5, P<$ \\
0.001). All interaction terms were non-significant and we therefore removed them from the model. ${ }^{1}$ Significance \\
test not indicated because of not having a meaningful interpretation.
\end{tabular}


a)

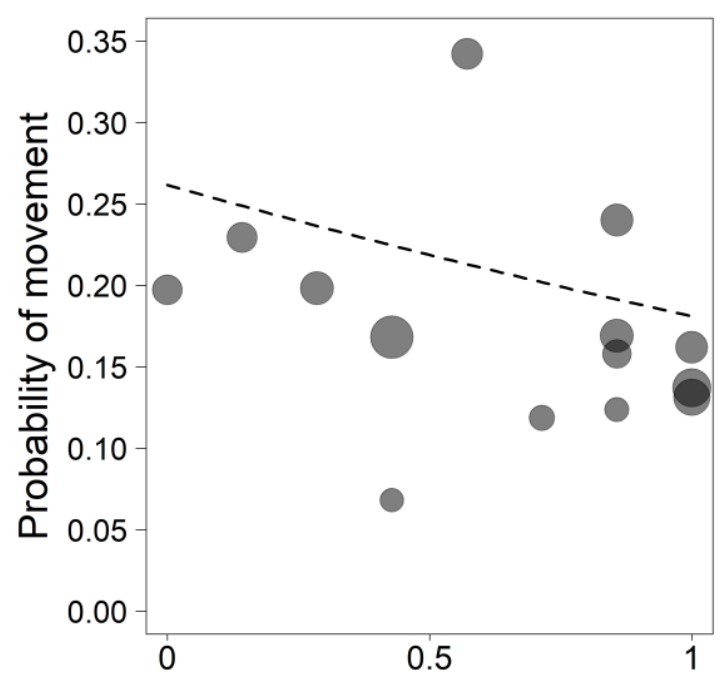

Increasing female dominance rank b)

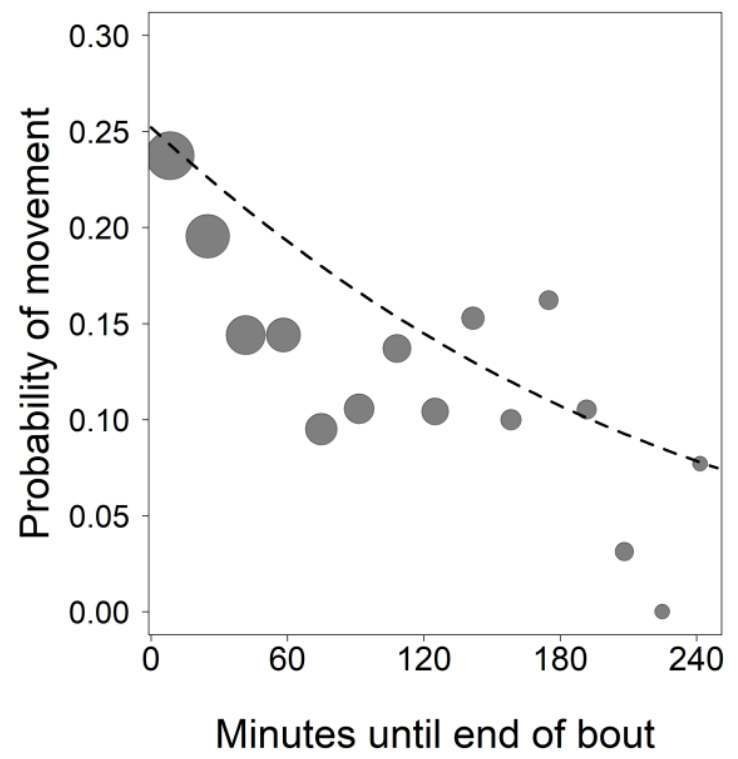

Figure 3: Probability of movement in focal patches as a measure of feeding effort in relation to a) female dominance rank (GLMM: estimate $\pm \mathrm{SE}=-0.150 \pm 0.069, \mathrm{X}^{2}=3.89 \mathrm{df}=1, P=0.049$ ), b) how many minutes remain until the end of the focal patch feeding bout (GLMM: estimate \pm SE $=$ $0.261 \pm 0.008, X^{2}=6.65, d f=1, P=0.010$ ). The area of each circle in $a$ ) is in proportion to the number of data points per female. The area of each circle in $b$ ) is in proportion to the number of data points across food type. The dashed line in both a) and b) depicts the fitted model.

Our behavioral models suggest that increasing dominance rank is associated with increasing food intake rate and reduced feeding effort when co-feeding. Next, we investigated whether these effects translated in to variation in energy balance by modelling uCP levels as a function of social and ecological factors. For the $N=218$ urine samples included in the analyses, the median uCP level per individual $(N=14)$ ranged from 1.49 to $3.30 \mathrm{ng} / \mathrm{mg}$ Crea with an overall range of values from 0.39 to $11.12 \mathrm{ng} / \mathrm{mg}$ Crea.

The full energy balance model was significantly different from the null model $\left(x^{2}=\right.$ 20.029, $\mathrm{df}=10, P=0.029$, Table 5). None of the interaction terms of dominance rank with a possible predictor of contest competition intensity was a significant predictor of urinary Cpeptide, and neither was dominance rank as a main effect in the reduced model (Table 5). The sole significant predictor of UCP levels was the monthly proportion of fruit in the diet as a measure of the seasonal abundance of these high quality resources (Figure 4). 
Table 5: Urinary c-peptide level increases as the monthly average proportion of fleshy fruits in the diet increases. Results are an overview of a LMM ( $N=218$ urine samples), with the significant effect displayed in bold.

\begin{tabular}{lcccc}
\hline & $\begin{array}{c}\text { Descr. Stats. } \\
\text { mean (range) }\end{array}$ & Estimate \pm SE & $t$ & $P$ \\
\hline Intercept & & $0.561 \pm 0.186$ & 3.012 & 1 \\
Mean party size & $9.0(3.0-20.7)$ & $0.063 \pm 0.061$ & 1.022 & 0.311 \\
\# food patches per hour & $0.70(0.16-2.89)$ & $-0.061 \pm 0.042$ & -1.457 & 0.150 \\
Proportion feeding scans & $0.39(0-0.88)$ & $-0.022 \pm 0.049$ & -0.448 & 0.674 \\
Rank & & $-0.052 \pm 0.065$ & -0.792 & 0.431 \\
Meters travelled per hour & $281.9(5.1-1083.8)$ & $0.049 \pm 0.050$ & 0.978 & 0.346 \\
Proportion of THV scans & $0.13(0-0.43)$ & $0.059 \pm 0.051$ & 1.145 & 0.266 \\
Monthly average proportion of fruit scans & $\mathbf{0 . 6 8 ( 0 . 3 8 - 0 . 8 8 )}$ & $\mathbf{0 . 1 7 4} \pm \mathbf{0 . 0 4 3}$ & $\mathbf{4 . 0 7 5}$ & $<\mathbf{0 . 0 0 1}$ \\
\hline
\end{tabular}

The full model was highly significant as compared to the null model (likelihood ratio test $\mathrm{X}^{2}=20.029, \mathrm{df}=10, P$ $<0.029$ ). All interaction terms were non-significant and we therefore removed them from the model. ${ }^{1}$ Significance test not indicated because of not having a meaningful interpretation.

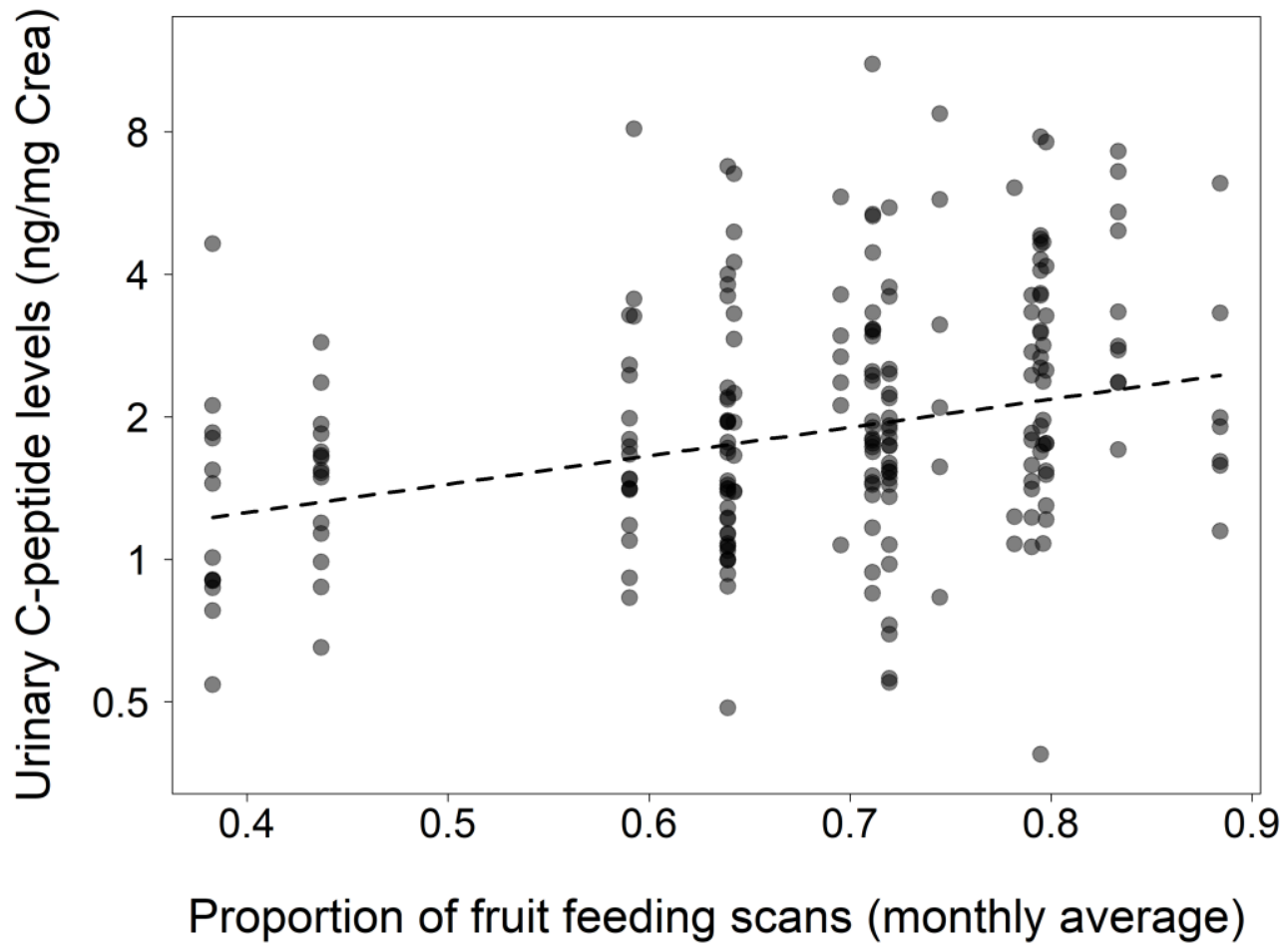

Figure 4: The effect of monthly average proportion of fleshy fruit on urinary C-peptide levels (LMM: estimate $\left.\pm \mathrm{SE}=0.174 \pm 0.043, X^{2}=11.29, \mathrm{df}=1, P<0.001\right)$. Urinary C-peptide levels are logtransformed. The dashed line depicts the fitted model. 
Female dominance rank and the average party size she travelled in were not correlated (Pearson's $r=0.12, N=8, P=0.78$; Fig. 5a); maximum (25) and minimum party size (1 to 3) were nearly identical for all females. There was also no rank effect on how often females were missing from group scan observations (Pearson's correlation between number of scan observations and female dominance rank $r=0.19, N=14, P=0.52$ ). At least one individual visited a THV patch in $7.2 \%$ of all group scans. Dominance rank did not have an effect on the time a female spent feeding on THV while travelling (Pearson's correlation between proportion of scans with THV feeding and female dominance rank $r=0.16, N=14, P=$ 0.59; Fig. 5b).

a)

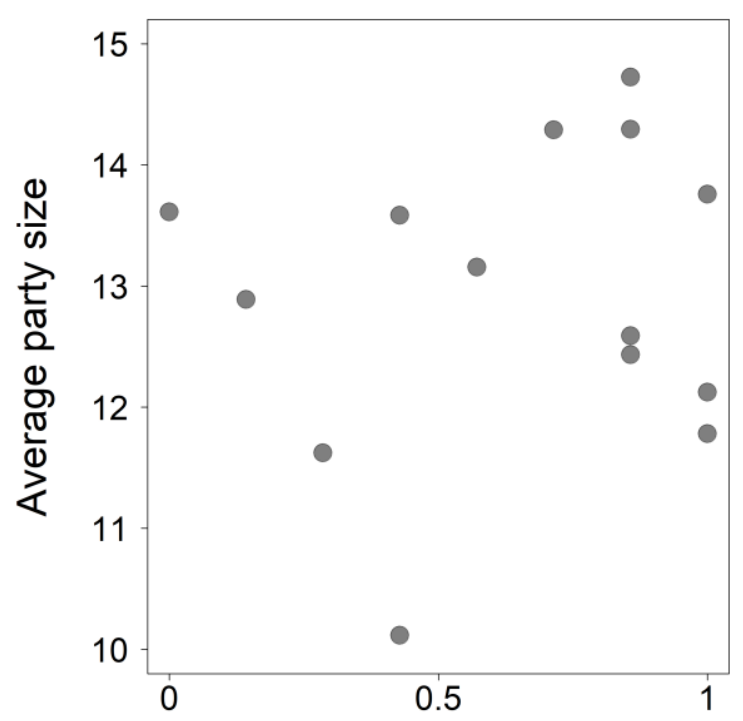

Increasing female dominance rank b)

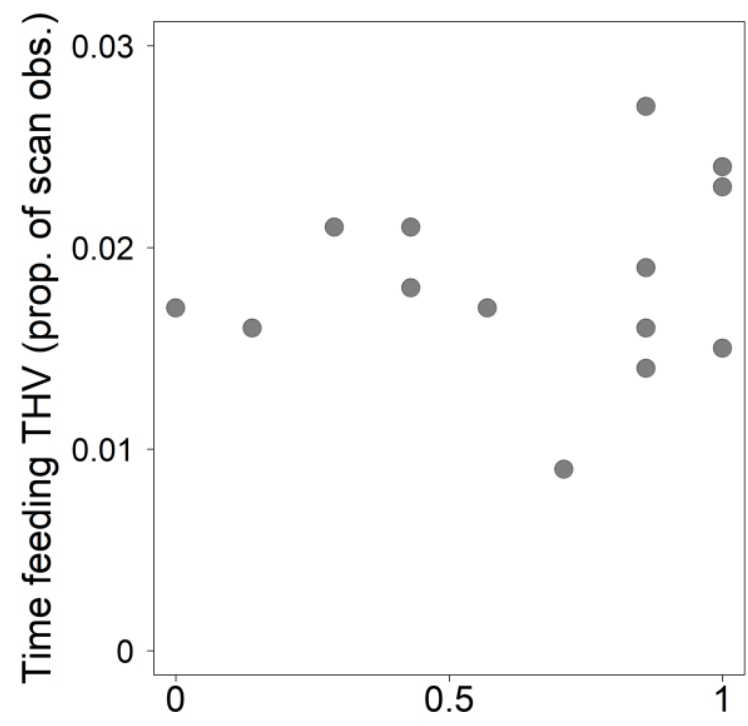

Increasing female dominance rank

Figure 5: Relation between female dominance rank and a) average party size the female was seen in (Pearson's $r=0.12, N=8, P=0.78$ ), and $\mathbf{b}$ ) time spent feeding terrestrial herbaceous vegetation $(\mathrm{THV})$, as the proportion of THV feeding scans over all scan observations including each female $(r=$ $0.16, N=14, P=0.60)$.

\section{DISCUSSION}

Our study revealed that females cooperated against male targets in agonistic coalitions, increasing female dominance rank was associated with decreasing feeding effort but moderate increases in food intake rates across a range of different food items and tree 
species, and that these rank effects did not translate into rank effects on female energy balance. Instead, variation of energy balance reflected the proportion of fruit in the diet with changes in UCP being independent of rank. In the following, we discuss these results in light of the three socio-ecological hypotheses for the evolution of bonobo female sociality and agonistic behavior.

The key prediction of the THV sub-hypothesis is that consumption of terrestrial herbs (THV) compensates for differences in food intake in fruit trees and that differences in access to such herbs are causally linked to the species-differences in female sociality (Wrangham 1986). However, in our study the use of THV was neither associated with dominance rank nor did dominance rank predict how often females were missing from the main party (our proxy to signal that subordinates would have opted to feed alone more often). A lack of rank effect on party size could be explained by rank assortative association in two or more parties of rather homogenous ranks. As strong evidence against such assortativeness, we have shown elsewhere that neither the social nor the rank relationship between females predicted their post fission association (Moscovice et al. 2017).

It has been suggested that rather than feeding on THV when others consume fruit, it could be that low ranking females compensate for the lower energy gain from fruit trees by feeding on THV when parties travel between fruit trees (Wrangham et al. 1996). The premise of this feed-as-you-go hypothesis is that either THV is uniformly distributed or that that patches are large enough to accommodate large parties, two conditions that are not always met (Malenky et al 1994). The model also suggests that unlike chimpanzees, bonobos slow down travel velocity when moving between fruiting trees (Wrangham 2000) which implies that most party members consume THV. However, in this case, it is reasonable to infer that the energetic gain from THV would be similar across individuals and that low ranking females would gain extra energetic benefits only if they had higher food intake or profit from enhanced fiber digestibility. Individual focal follows of females, including combined data on THV intake rates and food patch residence times, could yield a more rigorous test for potential rank effects on THV feeding. To specifically target potential compensation processes, comparative data are needed from females temporally leaving the party and those maintaining close proximity to others.

The key premise of the fruit patch size sub-hypothesis is that bonobos feed in fruit trees large enough to avoid direct and indirect feeding competition (Badrian and Bardian 1984, White 1986). Support for this is restricted to one data set (White and Wrangham 
1988), with subsequent studies finding that both Pan species use fruit tree patches of comparable sizes (e.g. Chapman et al. 1994, Furuichi et al. 2015). Our data also speak against the fruit patch size hypothesis because we found diminishing returns coupled with increasing effort suggesting that bonobos depleted fruit patches independent of their size, which evidently caused competition. Our behavioral data clearly show rank effects on female food intake and feeding effort, suggesting that females engage in contest competition over food. These rank effects should not prevail under the Resource Abundance hypothesis if competitive exclusion had no benefit (energy balance not affected by dominance rank) but carried any costs for the dominants over evolutionary time. If our assumption about contest effects fading over evolutionary time is wrong, then the Resource Abundance hypothesis would predict that subordinates would compensate for ingestion deficits in fruit patches by THV consumption, which was not the case either.

In support of the Cooperative Defense hypothesis and in agreement with earlier studies (Parish 1996, Surbeck and Hohmann 2013, Tokuyama and Furuichi 2016), female agonistic collaboration targeted mainly males and this was not a byproduct of females being generally more aggressive towards males than females, because females targeted males and females equally often in dyadic conflicts. Further support for the Cooperative Defense hypothesis came from the observation that food patches were depleted by feeding parties, and that dominance rank effects on food intake and feeding effort in fruit patches were moderate. The one prediction not supported by our data is that energy balance should vary with dominance rank. This could indicate either that high-ranking female bonobos do not profit through contest competition in terms of energy balance, or that effects exist but are too small to be detected by the analysis. Yet, we were able to detect individual changes in UCP between gestation and other reproductive phases. We also detected a nutritional signal with significantly higher uCP levels on days when bonobos mainly fed on abundant, easy to harvest, high sugar fruits versus days when feeding mainly on difficult to harvest starch rich fruits. Accordingly, the only significant predictor of uCP levels in our energy balance model was the proportion of fleshy fruit in the monthly diet. As a further point supporting the sufficient sensitivity of our energy balance measure, we have previously shown for the males of the same bonobo group that dominance rank effects on UCP can be picked up, but depend on the size of the party a male was travelling in, which can be interpreted as a proxy for food abundance (Surbeck et al. 2015). Another possibility is that high-ranking females indeed had energetic advantages from contest competition, but immediately transferred these gains to their offspring. If dominant females provided more or richer milk, 
differences would not be expected in female energy balance but rather in inter-birth interval or offspring survival. Our combined findings bear resemblance to the pattern observed in female mountain gorillas; despite clear behavioral indications of feeding competition (Wright et al. 2014, Wright et al. 2015, Grueter et al. 2016), uCP did not vary with dominance rank (Grueter et al. 2014). Future studies should try to link behavioral and energetic indicators of feeding competition more directly to assess whether UCP variation is more pronounced on days with more pronounced behavioral variation in terms of feeding times, ingestion rates and food quality.

The Priority of Access hypothesis predicts that female-female coalitions and female agonistic support in the feeding context should target other females, that dominance rank has strong effects on food intake and feeding efficiency in fruit patches, and on overall energy balance. Rank effects on food intake and feeding effort were significant across a wide range of different food items from many different species with largely varying resource characteristics, but these effects were generally weak. This interpretation is supported by visual inspection of the data for specific, frequently consumed food items, none of which showed pronounced rank effects. If females associated with one another to exclude other females from food resources, as predicted by the Priority of Access hypothesis, large differences in food intake rates between such alliances should ensue. Consequently, subordinates should be excluded from food patches and parties, yielding highly differentiated association patterns. However, subordinates were not missing from parties more often than dominants and lower ranking individuals did not avoid travelling in larger parties. Socio-ecological theory would also predict females to form strong affiliative relationships with their cooperation partners (Sterck et al. 1997, Ostner and Schülke 2014). Agonistic support, however, is not correlated with the strength of dyadic affiliative relationships in female bonobos at LuiKotale (Moscovice et al. 2017). This is partially supported by findings from bonobos at Wamba, where female coalition formation is facilitated by being associated in the same party yet coalitions did not correlate with proximity or grooming (Tokuyama and Furuich 2016). Hence, female bonobos seem to choose partners for cooperation and affiliation (Hohmann et al. 1999) rather opportunistically.

Our predictions derived from the Cooperative Defense and the Priority of Access hypotheses do overlap to some extent. Both hypotheses received some support from the result that i) food resources were depleted by the bonobos, a prerequisite for feeding 
competition to occur (Snaith and Chapman 2005, Heesen et al. 2014), and that ii) dominance rank was a positive predictor of food intake and a negative predictor of feeding effort in food patches albeit we predicted strong effect from the Priority of Access Hypothesis and observed weak effects. Our findings are not so different from what has been described for female chimpanzees in terms of rank-effects on feeding efficiency (reviewed in Pusey and Schroepfer-Walker 2013). Lower-ranking females of the Gombe community spent significantly more time foraging, and consumed a lower quality diet compared to highranking females (Greengrass 2005, Murray et al. 2006). Female chimpanzees at Gombe tend to concentrate their use of the community range by establishing overlapping core areas, with high-ranking females occupying smaller, higher quality core areas than lowranking females (Murray et al. 2007). Differentiated use of the community range occurs also at Kanyawara where high-ranking chimpanzee females are significantly more likely to occupy core areas containing high quality forage, and tend to exhibit shorter inter-birth intervals and higher levels of ovarian hormones (Emery Thompson et al. 2007, Kahlenberg et al. 2008a). At Taï National Park, where female chimpanzees tend to be more sociable than is typically reported for chimpanzees, and where they use the entire territory, often in mixed parties, contest competition was found to increase with increasing number of competitors, or when food was monopolizable. In the latter case, high-ranking females possessed food significantly more frequently following a conflict than low-ranking females (Wittig and Boesch 2003).

Dominance rank effects on feeding behavior are highly variable within and between primate species independent of their broadly categorized diet. Among frugivores female olive baboons (Papio cynocephalus anubis) exhibit strong rank effects on supplant rates, food intake rates and energy intake (Barton and Whiten 1993, Barton 1993), whereas Assamese macaques (Macaca assamensis) exhibit none of these effects (Heesen et al. 2013). Dominance rank predicts energy intake in folivorous Hanuman langurs (Semnopithecus entellus, Koenig 2000) and rank effects are variable within folivorous Mountain gorillas (Gorilla beringei beringei). Dominance rank affects THV feeding site residence times in one population (Grueter et al. 2016) and fruit feeding time in another (Wright et al 2014), which translates into rank effects on energy intake/balance in one case but not the other. Together these results suggest that the drivers and consequences of feeding competition can only be understood with an integrated view combining detailed knowledge about food resource characteristics, differential feeding behavior within food patches, inter-individual variation occurring between patches, and a full picture of the 
energetic consequences of differential resource use and how it is traded-off against other benefits.

The prediction most clearly differentiating between the two hypotheses is that under the Priority of Access hypothesis female-female coalitions should mainly target other females whereas collaborative aggression should mainly target males under the Cooperative Defense hypothesis. We found the latter prediction to be supported. In dyadic conflicts on the other hand, females did not target males more often than females. Thus, it is not the use of force in general that differs according to target sex, but specifically the collaborative element that characterizes conflicts with males. Another prediction that differentiates between the two hypotheses was that only under the Cooperative Defense hypothesis all contest effects should be weakened by female-female tolerance that is required for their cooperation against males. With both of these predictions being met we conclude the Cooperative Defense hypothesis to be best supported by our data. This does not mean that cooperative defense of food sources against males is the only force promoting gregariousness in female bonobos. Females may associate to protect their offspring against males (e.g. lions, Packer et al. 1990), to defend the groups territory (e.g. spotted hyenas, Boydston et al. 2001), or to support close relatives (e.g. humans, Cant and Johnston 2008) or to maintain autonomy in mating partner choice (Wrangham and Pilbeam 2001, Hare et al. 2012, Hare 2017). In bonobos male infanticide has never been observed, but male aggression against immature individuals is likely to trigger agonistic aid among females (Surbeck and Hohmann 2013).

Other evidence in support of female bonobos counteracting aggressive male mating strategies includes extended periods and reduced precision of sexual signaling compared to chimpanzees (Douglas et al. 2016), and earlier maturation of females (Behringer et al. 2014) coupled with increased tolerance towards and early bonding with young immigrant females in bonobos versus delayed dispersal due to the resistance from residents in chimpanzees (Pusey 1990, Boesch and Boesch-Achermann 2000, Kahlenberg et al. 2008b). While gregariousness among female bonobos may derive multiple benefits, feeding competition remains a prominent factor that affects most or all resident females independent of their age, parity and kinship. Ours and the published results discussed here are most congruent with the Cooperative Defense hypothesis.

More data on intra- and intersexual conflicts, especially on polyadic conflicts, are needed and on other indicators of females excluding males from access to limiting 
resources. A more detailed understanding of female energy allocation to different functions (energy storage and maintenance functions versus investments in current reproduction) will be required until the Priority of Access hypothesis can be firmly rejected. Since another contrast between chimpanzees and bonobos concerns differences in male-male cooperation, one may also turn the argument around and, instead of asking why female bonobos exclude males from resources, ask why the highly cooperative male chimpanzees will never allow that. Thus, understanding the differences in female social relationships between bonobos and chimpanzees ultimately will require broadening the perspective to male-male and intersexual relationships.

\section{FUNDING}

This work was supported by Deutsche Forschungsgemeinschaft (DFG grants SCHU 1554/4-1 and HO 1151/9-1).

\section{ACKNOWLEDGEMENTS}

We thank the Institut Congolaise pour la Conservation de la Nature (ICCN) for granting the research permit along with the export permit for urine samples, the people of Lompole village for permitting to use their forest for research, Josephine Hubbard and Lieven DeVreese for assistance in the field, and Barbara Fruth for advice and support in the field and for the identification of botanic samples. We are grateful to Colleen Stevens and Roger Mundry for statistical analyses and advice, Róisín Murtaugh and Vera Schmeling for their expertise and help with endocrinology laboratory analyses, and Tina Knittel for help with data entry. We also thank Edward Wright, Cédric Girard-Buttoz, Marlies Heesen, Alexander Mielke, Verena Behringer and Martin Surbeck for helpful discussions at various stages of the project, and our two anonymous reviewers for their valuable comments to improve the manuscript.

\section{DATA ACCESSIBILITY}

Analyses reported in this article can be reproduced using the data provided by Nurmi et al. (2018) 


\title{
CHAPTER 2
}

\section{Effects of reproductive state and dominance status on urinary cortisol levels in wild female bonobos}

\author{
Niina O. Nurmi ${ }^{1,2}$, Gottfried Hohmann², Liza Moscovice ${ }^{3}$, Tobias \\ Deschner $^{2}$ and Oliver Schülke ${ }^{1,4}$ \\ ${ }^{1}$ Department of Behavioral Ecology, JFB Institute for Zoology/Anthropology, \\ University of Göttingen, Germany, ${ }^{2}$ Department of Primatology, Max \\ Planck Institute for Evolutionary Anthropology, Germany, ${ }^{3}$ Anthropology \\ Department, Emory University, USA, ${ }^{4}$ Research Group Social Evolution in \\ Primates, German Primate Center, Leibniz Institute for Primate Research, \\ Göttingen, Germany.
}




\section{ABSTRACT}

Living in social groups entails costs arising from feeding competition and social conflict. The concept of allostatic load provides a useful framework to investigate how nutritional stressors arising from increased feeding competition and social stressors arising from unpredictable conflicts with conspecifics challenge the maintenance of homeostasis of individuals. Glucocorticoids are a common proxy of allostatic load. Animals living in social groups often form dominance hierarchies and hence dominant or subordinate group members may experience different levels of allostatic load (stress of subordination, stress of domination). In this study we examined urinary cortisol levels as a hormonal measure of allostatic load in female bonobos (Pan paniscus) to assess if a significant portion of interindividual variation in cortisol levels could be explained by dominance rank and reproductive state. Unlike in many other primate species, female bonobos disperse from natal communities and dominance rank position acquisition does not depend on maternal support. Bonobo females are co-dominant with males, and increased female gregariousness is thought to facilitate female-female coalitions in conflicts against males. Females provide agonistic support for their sons in male-male conflicts over access to mating partners. This makes female bonobos a particularly interesting system to investigate the costs of gregariousness by assessing the relative effects of competition for food and competition for mating partners on female cortisol levels.

\section{KEYWORDS}

dominance, glucocorticoids, Pan paniscus, allostatic load 


\section{INTRODUCTION}

Gregarious animals can benefit in many ways from living in groups including enhanced acquisition and defense of food resources, increased safety from predators, reduced risk of infanticide, and social immunity (Alexander 1974, Wrangham 1980, van Schaik 1996, Meunier 2015). These benefits of gregariousness are diminished by the costs of competition for limiting resources within groups, which include food, safety, and access to mating partners (Alexander 1974, Wragham 1980, Bradbury and Vehrencamp 1977). The costs of gregariousness will not be evenly distributed across group members if they contest over access to resources by interference which can include aggression, displacements and avoidance selecting for ritualized conflicts, displays, and dominance hierarchies to reduce the risk of escalated conflicts (Maynard Smith and Price 1973, Maynard Smith 1974).

Provided the sexes differ in their potential reproductive rates (Ahnesjö et al. 2001) they will differ in within sex variance in reproductive success and within sex competition for access to the sex with the lower potential reproductive rate will ensue (Andersson 1994). Among mammals, females carry the burden of lactation and gestation and typically have longer reproductive time-outs (Clutton-Brock and Parker 1992) constraining their potential reproductive rates which creates intrasexual competition among males. Thus, the key resources males and females compete over can differ (Trivers 1972, Cain and Rosvall 2014). Since females increase their reproductive success by maximizing net energy intake for the production and nurture of offspring, for females the immediate benefit of winning a conflict over food will outweigh the benefit of getting access to a specific mating partner (Stockley and Bro-Jorgenson 2011). Here, we aim at assessing the costs of gregariousness for female bonobos.

Bonobos live in multi-male multi female communities with a high degree of fissionfusion dynamics. Hence, communities can split in to smaller temporary parties that can vary in duration, composition and size (Furuichi 2011). Because most female bonobos disperse from their natal community there is no strong relatedness structure among the females (Gerloff et al. 1999, Moscovice et al. 2017). Dominance hierarchies of males are steeper and more linear than among females and some females can outrank males (Surbeck et al. 2012, Surbeck and Hohmann 2013). Females contest over food as evident from moderate effects of dominance rank on food intake rates, which however do not translate into variation in energy balance (Nurmi et al. 2018). The rate of aggression is low among females compared to males or the congeneric female chimpanzee (Furuichi 2011), aggression does 
not increase in the feeding context, and in dyadic conflicts aggression is directed mostly from long tenured females towards females with the shortest shared tenure i.e. recent immigrants (Moscovice et al. 2017). Females cooperate in conflicts against males but not against females (Tokuyama et al. 2016, Nurmi et al. 2018). Male mating success and reproductive success is affected strongly by the presence of their mother who fends off competitors by providing agonistic support (Surbeck et al. 2011, Surbeck et al. 2017). Therefore, female bonobos are an ideal case to study the dual costs of gregariousness that accrue from competition over access to food and access to mating partners.

The costs of gregariousness can be conceptualized as responses to stressors, which are stimuli that pose a challenge to, or threaten to challenge homeostasis (Romero et al. 2009). Homeostasis is the stability of physiological systems considered essential for sustaining life (McEwen and Wingfield 2003) and deviations from homeostasis cause allostasis which is the process of readjusting internal processes to reinstate homeostasis (McEwen and Wingfield 2003). Among the mediators of allostasis are glucocorticoids (GCs), the primary hormones of the hypothalamo-pituitary-adrenal (HPA) axis. One of the main metabolic effects of glucocorticoids is the mobilization of energy substrates from the peripheral deposits of lipids and proteins in muscles and adipose tissue for glucose production in the liver (Dallman et al. 2007) by inhibiting protein synthesis and stimulating glyconeogenesis and proteolysis (Peckett et al. 2011).

Activation of the HPA axis can be viewed as a one-size-fits-all reaction to many external or internal challenges to homeostasis (Beehner and Bergman 2017); GC levels increase in reaction to low blood sugar levels (Leakey et al. 1994) which may result from low food intake (Messer et al. 1995, Tomiyama et al. 2010) or increased demands during certain life history stages (Salfen et al. 2003, Jelincic et al. 2017). Moreover, GC levels increase in reaction to cold stress (Beehner and McCann 2008, Young et al. 2014), immediate threat from aggressive conspecifics (Wittig et al. 2015), and social isolation in social mammals (Smith and French 1997, Hawkley et al. 2012). As a consequence, it can be difficult to disentangle the sources of observed GC level variation among individuals. One solution is to assess energy balance together with GC levels by measuring insulin, another major regulator of glucose metabolism. The release of the pancreatic hormone insulin into the blood stream is regulated directly via blood sugar levels (Hedeskov 1980) and can be assayed by quantifying the C-peptide that is cleaved off proinsulin at transport into the blood stream (Bonser et al. 1984). Because insulin stimulates the conversion of 
glucose into glycogen (glycogenesis) for storage, its actions are considered as antagonistic to those of glucocorticoids, which inhibit glycogen synthesis and glucose uptake into muscle and adipose tissue to maintain higher levels of circulating glucose (Kuo et al. 2013). If GC levels are mainly driven by low blood sugar levels from low food intake and high demand, they should be negatively correlated to C-peptide levels. The stronger the effects of other stressors on GC levels, the weaker this correlation should be.

The allostatic load accruing from within-group competition can be assayed from correlations between GC levels and dominance rank (McEwen and Wingfield 2003). Depending on the processes by which dominance rank is attained (Beehner and Bergman 2017), the stability of dominance relationships, whether agonistic support is crucial for maintenance of dominance, and whether contests ensue mainly over access to resources or mainly over status (Goymann and Wingfield 2004), positive and negative correlations have been observed across vertebrates (Goymann and Wingfield 2004, Creel et al. 2013). Across primate species, GC levels of females tend to be negatively related with dominance rank, while in males the correlation tends to be positive (Beehner and Bergman 2017). Allostasis caused by food shortages or changing energetic demands is evident from negative associations of GC levels and food availability (Foley et al. 2001, Pride 2005, Berghänel et al. 2017) or changing GC levels depending on female reproductive state (Goymann et al. 2001, Hackländer et al. 2003). Energetic demands of female mammals increase from the cycling phase to gestation, during gestation towards birth, until peak lactation when energetic demands are maximal (e.g. Cervus elaphus, Clutton-Brock et al. 1989; Phoca vitulina, Bowen et al. 2001; Cebus capucinus, McCabe and Fedigan 2007; Pan troglodytes, Emery Thompson et al. 2012).

Here we contribute to disentangling costs of gregariousness accruing from energy restriction and increased demands from those resulting from mating competition in wild female bonobos. More specifically, we predict i) high GC levels for high-ranking females, because they cannot rely on the coalitionary support for dominance rank acquisition, and low GC levels for subordinates because they rarely receive aggression from dominants, ii) that the dominance effect on GC levels changes with the energetic demands of reproduction which are highest during peak lactation (Emery Thompson et al. 2010), and iii) that GC levels are negatively associated with C-peptide levels if allostasis is mainly caused by energetic shortage because C-peptides vary with food abundance in these females (Nurmi et al. 2018). In a post-hoc test, we assess whether C-peptide levels vary with reproductive 
state as a function of varying energy demands. To validate our urinary GC assay for female bonobos we test whether GC levels show the decrease from very high morning values throughout the day that is characteristic for humans and apes (Muller and Lipson 2003) and include time of day in our multivariate models.

\section{METHODS}

\section{Ethics statement}

Our protocols and methods followed a strict non-contact, non-invasive procedure. The Institut Congolaise pour la Conservation de la Nature (ICCN) granted us the permission to conduct research at LuiKotale, Salonga National Park, Democratic Republic of Congo (0683/ICCN/DG/ADG/014/KV/2012). Our permits for exporting the urine samples from the Democratic Republic of Congo were issued by the ICCN (0521/ICCN/DG/CWB/05/01/2014). The state ministry for social affairs and consumer protection of Saxony/Germany issued permits for importing the samples to Germany.

\section{Study site and subjects}

The LuiKotale field site is located close to the south-western edge of Salonga National Park, in Democratic Republic of Congo (Hohmann and Fruth 2003). The study site ( $2^{\circ} 47^{\prime} S, 20^{\circ} 21$ E) is contained within continuous equatorial rainforest comprised mainly of lowland tropical forest (Beaune et al. 2013b). Rainfall tends to be seasonal, with a long dry season spanning from June to August, and a short one around February. Our research was conducted between February 2012 and August 2014. During this period, we collected data on the fully habituated Bompusa West community, consisting of five adult and two subadult males, and 16 adult and three subadult females. Of the 16 adult females, 13 were parous females and three were nulliparous. Our subjects included 14 of the adult females, considered as resident in the community from the onset of the study.

\section{Behavioural observations}

We observed bonobos during half-day shifts ( $N=223$ days), either from the morning nest site until noon or from noon until the evening nest site. Most follows were conducted on party level due to the fission-fusion dynamics of the species. We define a party as a temporary subgroup of the community that varies in size, duration and composition of individuals throughout the day. Two observers collected the data working as a team with 
pre-assigned methods and consistent roles throughout each field season. Each observer carried a notebook and a Garmin GPS device. One observer recorded the party composition at each half hour interval ( $N=3644$ scans), and agonistic interactions on all occurrence basis by noting down the context of the interaction, along with the identities and roles of the opponents (Altmann 1974).

\section{Female dominance ranks}

A graph based method, ADAGIO, has been develop recently for constructing linear and nonlinear dominance hierarchies (Douglas et al. 2017). Agonistic interactions among female bonobos are relatively rare and some female dyads do not have any recorded dyadic agonistic interactions. We used an ADAGIO chart based on all dyadic decided overt and subtle agonistic interactions from our observation period to rank our study subjects into eight rank categories. Overt agonistic interactions included contact aggression such as biting, pulling and holding, and non-contact aggression included charges, chases and directed displays, whereas subtle interactions included displacements and threats. We calculated these categories based on the ADAGIO top-down method approach (Douglas et al. 2017). Each female at the root-nodes of the graph were assigned $r=1$, corresponding to the highest rank, and the direct child-nodes of these females were assigned $r+1$ etc.

\section{Reproductive state}

For each day we assigned each female to the following reproductive states: early lactation lasting from birth to 6 months ( $N=68$ samples); late lactation lasting from 6 months after parturition until two years of infant age; and cycling (females with infants older than 2 years. Because information on reproductive profiles of female bonobos is not yet available, we used corresponding information from chimpanzees (Emery Thompson et al. 2012, Emery Thompson 2013). Because of their low incidence $(N=30$ samples) and possible large effects (Duthie and Reynolds 2013), we excluded samples from pregnant females with pregnancy assessed backwards from birth and confirmed by pregnancy tests for 10 of the 14 individuals.

\section{Urine samples}

We collected 544 urine samples opportunistically from the 14 female bonobos throughout the study period (mean $\pm S D=37 \pm 15$, range $=13-66$ samples per individual). Most of the samples were collected using the underside of a large Marantaceae leaves. Alternatively, 
the urine was pipetted directly from the ground foliage $(N=35)$ or from a frisbee covered with a plastic bag $(N=62)$. All samples were collected on the condition that they had not come into contact with urine from another individual or in contact with feces (Higham et al. 2011a). Samples were stored in liquid nitrogen containers and later transported in dry ice to the Max Planck Institute for Evolutionary Anthropology in Leipzig, Germany, where upon arrival they were stored in $-20^{\circ} \mathrm{C}$ until hormone analysis.

\section{Cortisol extraction and measurement}

We extracted cortisol from urine samples according to the method described by Hauser et al. 2008. We deviated from the method by using testosterone- $\mathrm{d}_{3}$ (T2655, Sigma-Aldrich) as internal standard for measuring extraction efficiency, because the standard used by Hauser et al. (2008), prednisolone, coeluted at a retention time coinciding with an unknown substance. Before extraction, samples were thawed at room temperature, followed by 10 seconds of mixing with a vortexer (VX-2500, WWR), and centrifuged for 10 minutes at 4.400 rpm (Multifuge, Heraeus). After adding an internal standard mixture to each sample, we deconjugated the steroid glucuronides using enzymatic hydrolysis with ß-glucuronidase from Escherichia coli (G7646, Sigma), followed by a solvolysis step using ethyl acetate/sulphuric acid to cleave off steroid sulphate conjugates. We measured urinary cortisol levels using liquid chromatography-tandem mass spectrometry (Waters Alliance 2695 coupled to a Quattro Premier XE with a Z-spray ESI interface) with a method adapted from Hauser et al. (2008). Control samples with known concentration of cortisol were included among each measurement batch. We accepted batches with control sample measurements deviating less than 15\% from known cortisol concentrations. Extraction efficiency was calculated based on the recovery of internal standard testosterone- $d_{3}$. We corrected for variation in urine concentration by measuring creatinine in each sample using the colometric Jaffe method (Taussky 1954, Bahr et al. 2000) and indexing cortisol levels to creatinine. Therefore, we report all cortisol measurements as ng cortisol /mg creatinine. Urine samples with creatinine values of less than $0.05 \mathrm{mg}$ creatinine/ml were excluded from the analysis $(N=4)$.

\section{C-peptide extraction and measurement}

We measured C-peptide levels using solid phase Enzyme Amplified Sensitivity Immunoassay kits (DIAsource, C-Pep-EASIA KAP0401) on thawed urine samples $(N=446)$ that were centrifuged for 10 minutes (Heraeus Multifuge). The immunoassay has been 
validated for bonobo urine (Surbeck et al. 2015). Intra-assay coefficients of variation (CV) were based on the mean CVs of four replicates. The low-value quality controls were $3.89 \%$ and the high-value quality control were 3.75\%. Inter-assay CVs for low-quality controls were $2.66 \%$, and $8.85 \%$ for high-value quality controls ( $N=15$ plates). We indexed C-peptide levels by creatinine (crea) levels in each sample to adjust for variation in the concentration and volume of urine (Bahr et al. 2000). We therefore report the C-peptide measurements as ng C-peptide/mg crea. We omitted samples with a creatinine value lower than $0.05 \mathrm{mg}$ $\mathrm{crea} / \mathrm{ml}$ from further analyses $(N=2,0.84 \%)$. In addition, we excluded $N=1$ sample $(0.42 \%)$ because it did not yield detectable C-peptide levels.

\section{Statistical analyses}

We first examined the relationship between mean cortisol levels and dominance rank in 14 female bonobos using an exact Spearman correlation test ( $R$ function "cor.test") in $R$ version 3.4.3. (R Core Team 2017). For this and other analyses we scaled the ADAGIO based dominance ranks to range between 0 and 1 , with 0 for the lowest ranked female and 1 for the highest. In order to minimize the effect of diurnal cortisol secretion pattern we calculated mean cortisol levels for these correlations from 355 urine samples (range from 8 to 48 samples per individual) collected before $10 \mathrm{AM}$. To assess the degree to which the correlation was driven by the highest-ranking individuals, we repeated the test on a reduced data set of 251 samples (range per female: 8 - 46) excluding the three females that together occupied the highest rank position.

Across 491 samples, we assessed the influence of female dominance rank, reproductive state and their interaction on GC levels by fitting a Linear Mixed Model (LMM) with a Gaussian error structure (Baayen 2008). We included time of sample collection as a predictor because GC levels may decrease from the morning through the day (Surbeck et al. 2012). We log-transformed our response variable, urinary cortisol levels (standardized for creatinine), to obtain a more symmetrical distribution. We included time of urine sample collection as test variable to examine the presence of a predicted diurnal pattern of cortisol secretion (Muller and Lipson 2003, Anestis and Bribiescas 2004). Reproductive state and dominance rank were entered as categorical test variables; reproductive state with three levels (cycling, early lactation, late lactation) and dominance rank with two levels (highest rank position, all other rank positions). We included an interaction between dominance rank and reproductive state to account for the possibility, that rank might have a stronger effect on cortisol levels during specific reproductive states. Each test variable was centered to a 
mean of zero and a standard deviation of one using z-transformation to obtain comparable estimates and improved interpretation of results. We included female identity as a random effect to control for repeated measures (Schielzeth and Forstmeier 2009, Barr et al. 2013). We were not able to add a random slope of reproductive state within female ID because this lead to stability issues with the model, caused by singularity (Darlington and Hayes 2017, page 534). In other words, we needed to simplify the random effect structure due to the lack of variation within females to account for each level of the reproductive state. We determined Variance Inflation Factor (VIFs) for the standard linear model, excluding interaction and random effects, to check for potential multicollinearity issues among the predictors using the function "vif" (R package "car"; Fox and Weisberg 2011). We did not detect any issues with collinearity (maximum VIF=2.04). We established significance of the full model (Forstmeier and Schielzeth 2011) using a likelihood ratio test (Dobson 2002) by comparing it with the respective null model containing only the intercept and the random effect. On the condition that the full model was significantly different from the null model, we proceeded to test for the significance of the interaction term by comparing the full model with a reduced model in which rank, reproductive state and time of sample collection were included as main effects. If the interaction term was not significant, we continued with the reduced model by testing the significance of each test variable one at a time (Barr et al. 2013) using the "drop1" function in R.

We assessed the relationship between C-peptide of insulin and GC levels each measured from the same urine sample across 322 samples collected before 10AM from non-pregnant females in a second LMM with Gaussian error structure (Baayen 2013) with log-transformed GC levels as the response, urinary C-peptide as the sole predictor, and ID as a random factor. To maintain type I error at the nominal levels of $5 \%$, we added C-peptide level as a random slope component within the random effect of female ID (Barr et al. 2013). As a test of significance, we compared the full model (Forstmeier and Schielzeth 2011) with the respective null model (intercept and random effect only) using a likelihood ratio test (Dobson 2002).

As a post-hoc test to check for the relationship between C-peptide and reproductive state, we ran an LMM with log-transformed urinary C-peptide levels as the response variable ( $N=423$ samples from non-pregnant females). We entered reproductive state as the categorical predictor variable, and categorical binary dominance rank and sampling time as control variables. We added reproductive state as a random slope component within the 
random effect of female ID (Barr et al. 2013). There were no issues with collinearity (maximum VIF=2). We tested the significance of the model by comparing the full model (Forstmeier and Schielzeth 2011) with the respective null model (binary dominance rank, sampling time, and random effect only) using a likelihood ratio test (Dobson 2002).

All LMMs were fitted in R version 3.4.3 (R Core Team 2017) using the function "Imer" (R package "Ime4"; Bates et al. 2015) with the threshold for statistical significance set at $P$ $=0.05$. We visually inspected quantile-quantile plots and distribution of residuals plotted against fitted values to check that the assumptions of normally distributed and homoscedastic residuals were met; no signs of deviation from these assumptions were found. Model stabilities were checked by omitting each level of random effect one at a time and comparing the derived model estimates with those obtained from the full model. We did not find any influential cases based on these comparisons.

\section{RESULTS}

Urinary cortisol levels followed a distinct diurnal pattern; cortisol levels were highest in the early morning and declined steadily towards the early evening hours (estimate $\pm S E=-0.80$ $\pm 0.07, P<0.001$; Table 1, Fig 1).

Table 1. Predictors of urinary cortisol levels ( $N=491$ samples) in female bonobos $(N=14$ females). Due to a non-significant interaction between reproductive state and binary rank $\left(X^{2}=3.4, \mathrm{df}=2, P=\right.$ $0.185)$, the results are from a reduced model excluding the interaction. The reduced model was significant compared to the null model $\left(X^{2}=167.8\right.$, df $\left.=6, P<0.001\right)$. Cortisol levels were logtransformed. Time, reproductive state and rank were z-transformed. Significant effects are highlighted in bold.

\begin{tabular}{lrrr}
\hline Effect & Estimate \pm SE & $X^{2}$ & $P$ \\
\hline (Intercept) & $3.84 \pm 0.07$ & 1 & 1 \\
Time of sample collection & $-0.80 \pm 0.07$ & 128.38 & $<0.001$ \\
Reproductive state (early lactation) & $0.57 \pm 0.11$ & 24.39 & $<0.001$ \\
Reproductive state (mid lactation) & $0.19 \pm 0.09$ & & \\
Dominance rank (highest vs all & $0.44 \pm 0.10$ & 12.61 & $<0.001$ \\
other) & & &
\end{tabular}

${ }^{1} P$ - value omitted because has no meaningful interpretation 


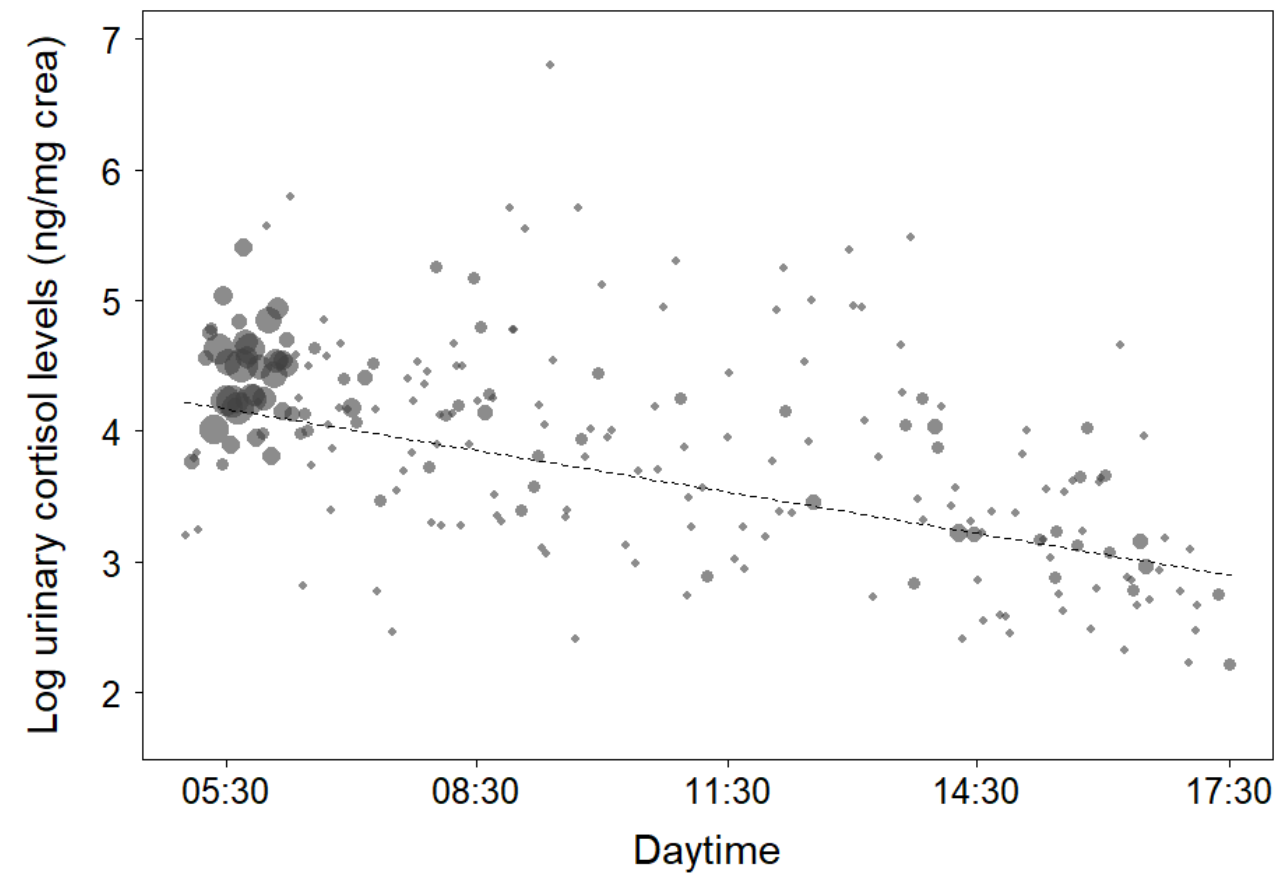

Figure 1. The effect of time of sample collection on urinary cortisol levels $(N=491)$. Time of sample collection had a significant effect on cortisol levels which showed a clear diurnal pattern of higher levels in the morning and declining towards the evening (estimate $\pm \mathrm{SE}=-0.80 \pm 0.07, P<0.001$ ). Cortisol levels are shown on log-scale. The size of data points represents the number of urine samples obtained at different daytimes.

Individual average cortisol levels ranged between 80.6 and $192.8 \mathrm{ng} / \mathrm{mg}$ creatinine and were predicted by social status among the 14 female bonobos (Spearman's rank correlation; rho $=0.63, P=0.017$; Fig $2 \mathrm{a}$ ). This relationship was driven partly by the high cortisol levels of the three top ranked females; cortisol and dominance rank were not associated strongly across the non-alpha females (Spearman's rank correlation; rho $=0.29$, $P=0.38$; Fig $2 \mathrm{~b}$ ) and we treated dominance as a dichotomous variable in the other analyses. 
a)

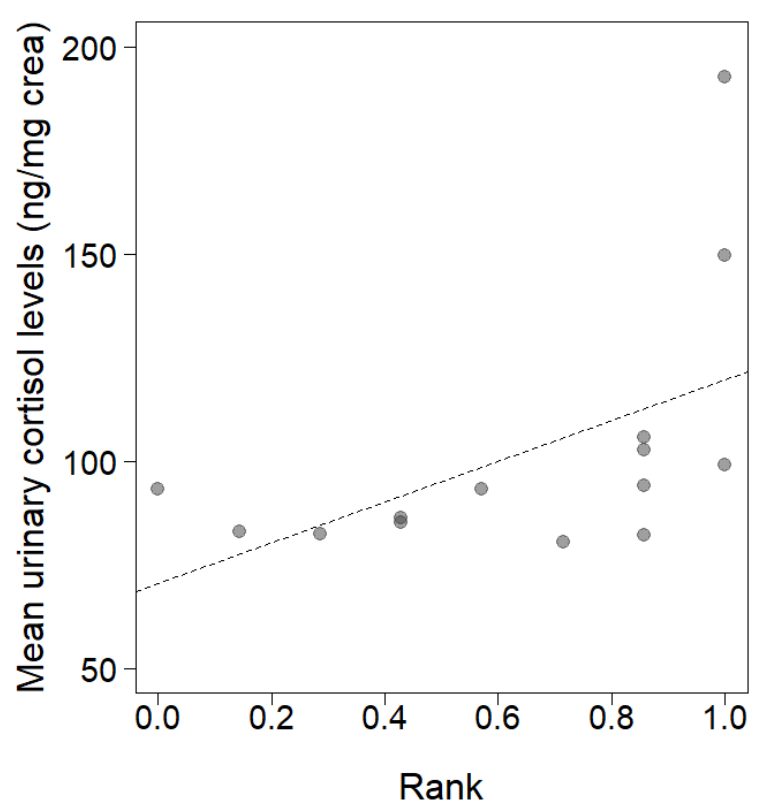

b)

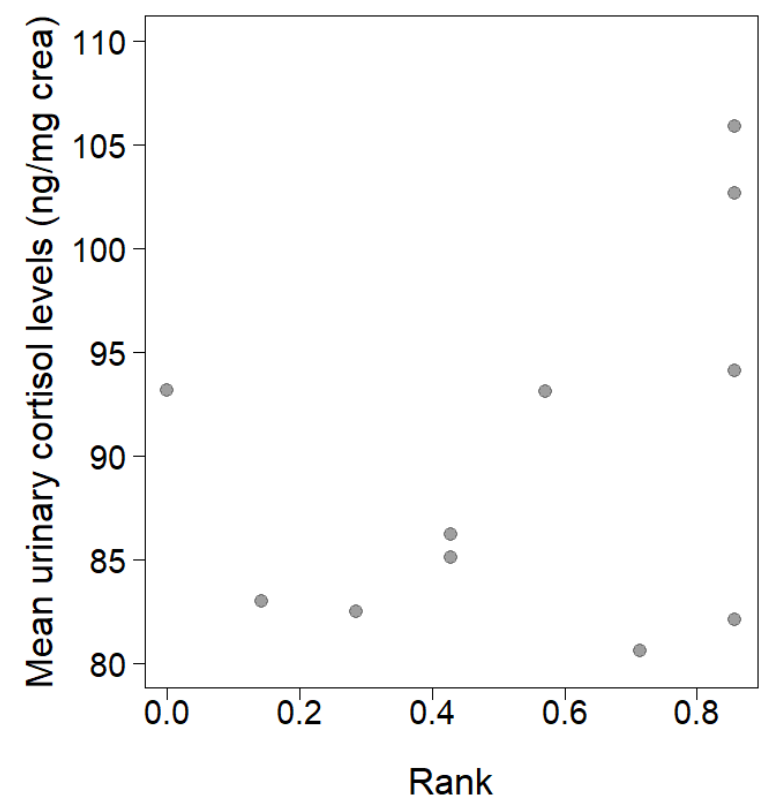

Figure 2. The correlation between mean cortisol levels (standardized for creatinine) and dominance rank is a) significant with all ranks included $(N=14$; Spearman's rank correlation; rho $=0.63, P=$ $0.017)$, and b) not significant when excluding three highest ranked females ( $N=11$; Spearman's rank correlation; rho $=0.29, P=0.380$ ).

In order to test for the costs of dominance in female bonobos, we examined whether variation in urinary cortisol levels (range 5.3-899.4 ng/mg creatinine) was explained by binary dominance rank position (highest dominance rank vs all other dominance ranks) controlled for reproductive state (cycling vs early lactation vs late lactation) and their interaction. Dominance effects did not change with reproductive state; the interaction term between the two variables did not affect significantly the variation in cortisol $\left(x^{2}=3.4\right.$, $d f=$ $2, P=0.185)$. After removing the interaction from the full model, we ran a reduced model to establish $P$ - values for the main effects (Table 1 ). Binary dominance rank affected urinary cortisol levels of females in the sense that the three alpha females had elevated cortisol levels compared to the eleven females on all other rank positions (estimate \pm SE $=0.44 \pm$ $0.10, P<0.001$; Fig 3a). Female reproductive state had an independent effect. Cortisol levels were highest in females during early lactation (estimate $\pm S E=0.57 \pm 0.11, P<0.001$; Fig $3 b$ ). Later lactation was associated with slightly higher cortisol levels (estimate $\pm \mathrm{SE}=$ $0.19 \pm 0.09)$ compared to cycling females. 
a)

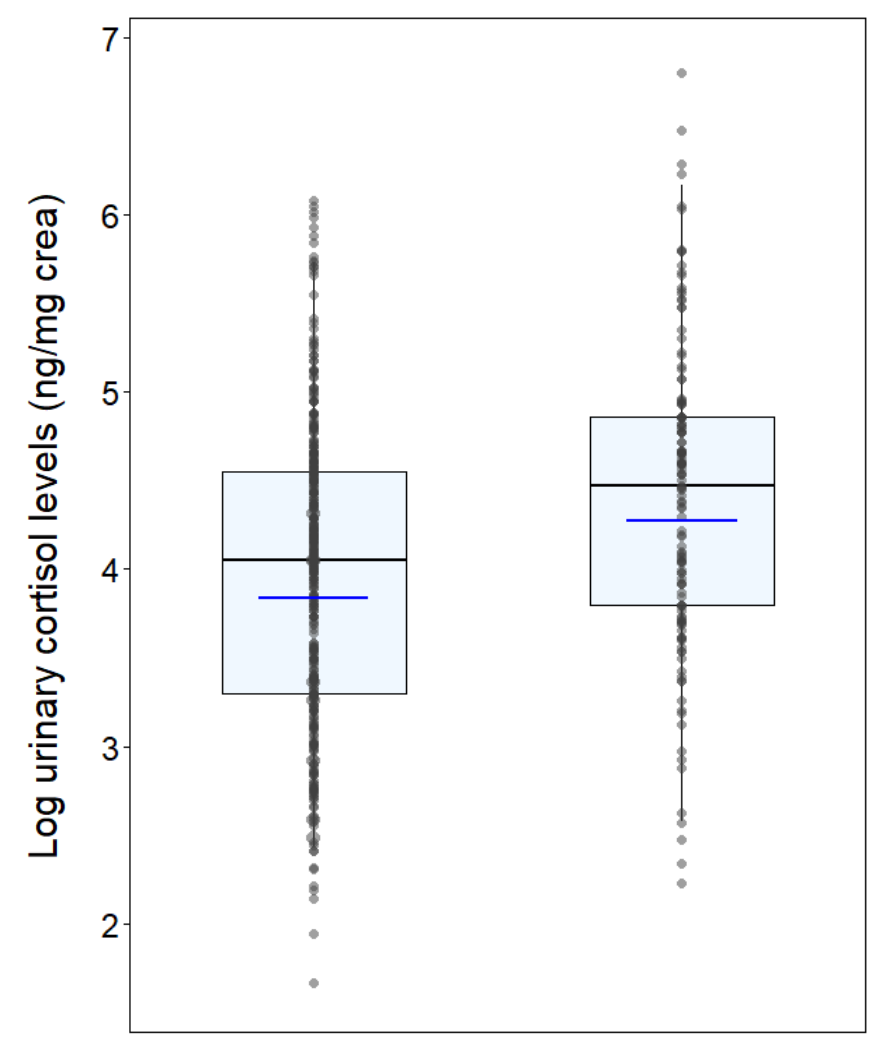

Other dominance rank Highest dominance rank b)

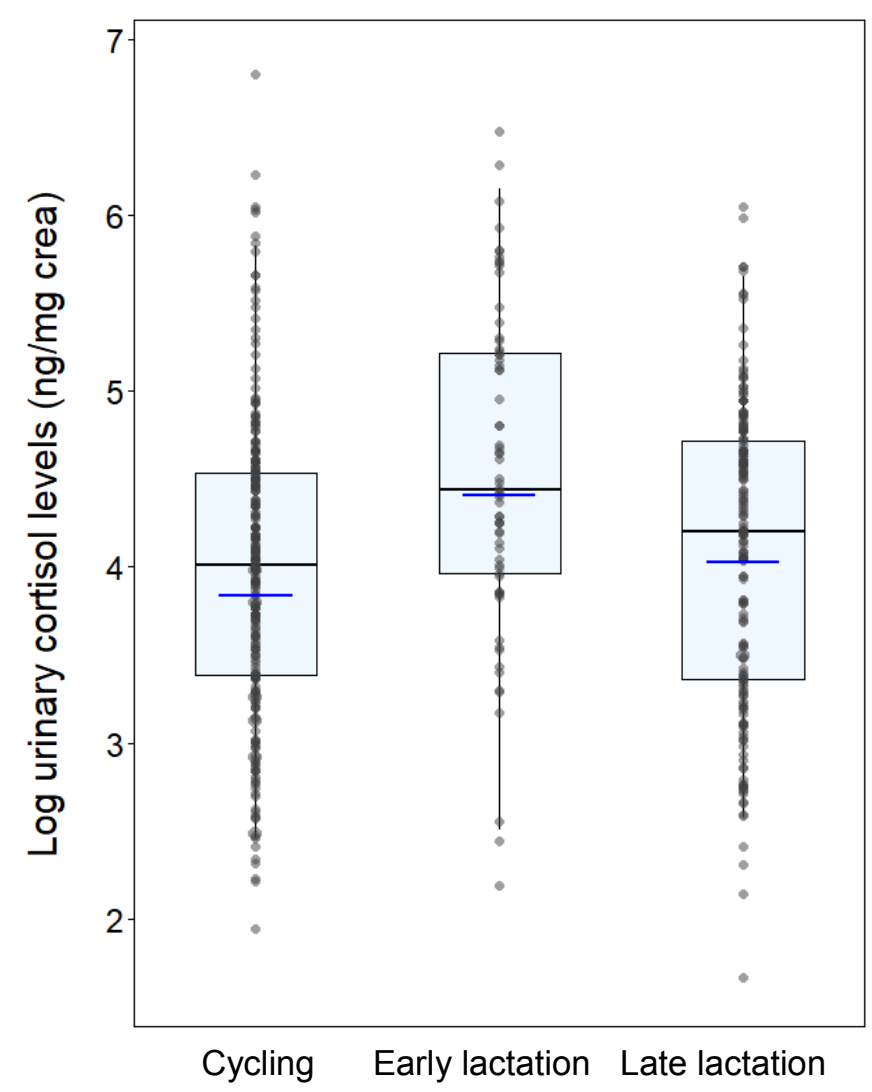

Figure 3. The effect of a) binary dominance rank (estimate $\pm \mathrm{SE}=0.44 \pm 0.10, P<0.001$ ), and b) reproductive state (estimate $\pm \mathrm{SE}=0.57 \pm 0.11, P<0.001$ ) on urinary cortisol levels $(N=491)$. The narrower blue line inside boxes indicates model line obtained from fixed effects. The wider black line indicates mean of individual cortisol level values. Data points are the individual cortisol level values. Box is drawn around the upper $(75 \%)$ and lower $(25 \%)$ quantile. Both urinary markers are standardized for creatinine. Cortisol levels are shown on log-scale.

For a subset of 322 samples we also quantified the C-peptide of insulin as a metabolic marker of energy balance (range $0.4-13.3$, mean \pm SD of $2.5 \pm 1.9 \mathrm{ng} / \mathrm{mg}$ creatinine. The urinary levels of cortisol and C-peptide in the same sample were not significantly associated $\left(X^{2}=0.79, \mathrm{df}=1, P=0.38 ;\right.$ Fig 4$)$. In a post-hoc test, urinary C-peptide levels of cycling females, females in early lactation state, or females in later lactation state were not different (non-significant full model against the null model: $X^{2}=2.1483$, df $=2, P=0.3416$; Fig 5). 


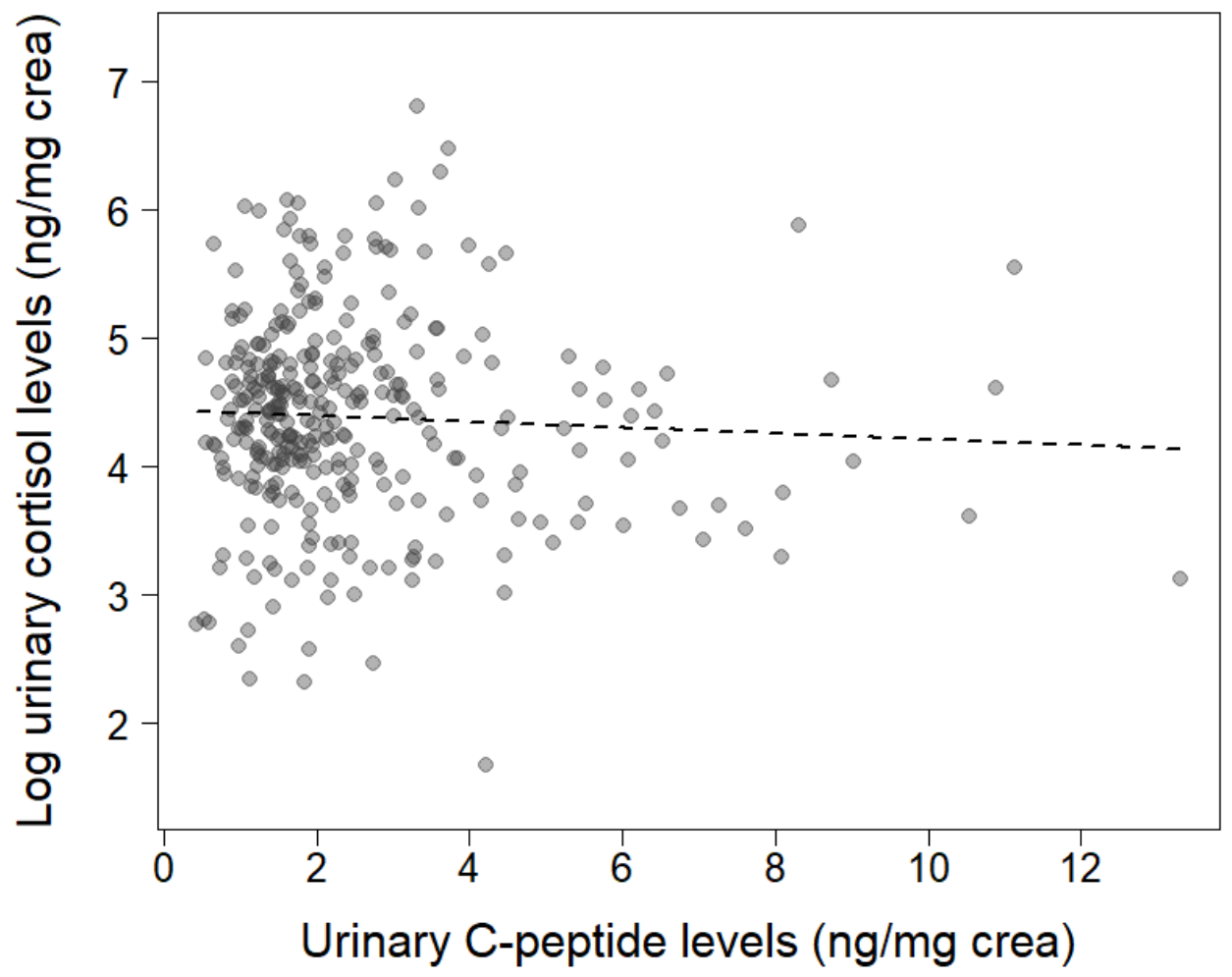

Figure 4. The effect of urinary C-peptide of insulin levels on urinary cortisol levels $(N=322)$. Cpeptide levels do not have a significant effect on cortisol levels when measured from the same urine sample $\left(X^{2}=0.79, \mathrm{df}=1, P=0.38\right)$. Both physiological markers are standardized for creatinine. Cortisol levels are shown on log-scale. 


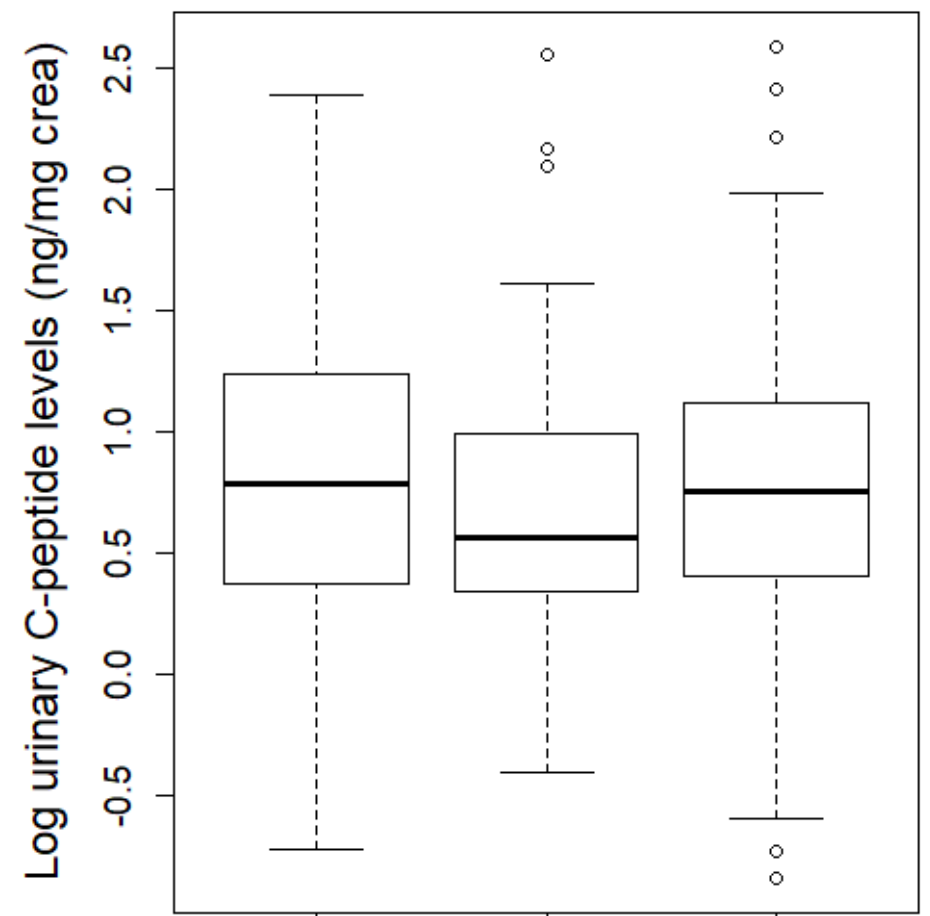

Cycling Early lactation Late lactation

\section{Reprodutive state}

Figure 5. The effect of reproductive state on urinary C-peptide of insulin levels $(N=423)$. Reproductive state does not have a significant effect on C-peptide levels $(\mathrm{X} 2=2.1483$, $\mathrm{df}=2, P=$ 0.3416). C-peptide levels are standardized for creatinine and shown on a log-scale. Box is drawn around the upper (75\%) and lower (25\%) quantile, and the thick vertical line represents mean Cpeptide values.

\section{DISCUSSION}

The costs of gregariousness can differ between the sexes if, due to sexual selection, females mainly compete over access to food resources and males mainly over access to more slowly reproducing females. Here we used variation in urinary GC levels to measure intra- and inter-individual differences in allostatic load and assess the relative importance of feeding and mating competition in female bonobos. Allostatic load varied with female reproductive state, likely mirroring changes in energy demands and independent of these demands was higher in top-ranking compared to other females. Moreover, allostatic load did not vary with energy balance and energy balance did not vary with reproductive state. By exclusion, this suggests that allostatic load for dominant female bonobos may be driven 
more by mating competition, because rank effects on GC levels cannot be explained by variation in energy balance. Ideally, we would have employed multivariate statistical modelling to test these interdependencies with GC levels as a response and C-peptide levels, dominance rank, reproductive state and their interactions as predictors. Yet, all models we built were unstable leaving us with the tests as described above.

Several studies have documented the distinct diurnal oscillation of plasma cortisol levels in humans (Krieger et al. 1971, Vagnucci et al. 1974, Debono et al. 2009) and nonhuman primates (Plant 1981, Smith and Norman 1987), with peak levels recorded in the morning and steadily declining levels towards the evening. This pattern reflects the circadian rhythm of cortisol secretion from the adrenal, which is endogenously governed by a central circadian oscillator located in the hypothalamus (Balsalobre et al. 2000, Chan and Dedono 2010). Our results are consistent with these and other studies using urinary cortisol (van Schaik et al. 1991, Czekala et al. 1994, Muller and Lipson 2003) as we also found higher and more variable urinary cortisol levels in the morning compared to the afternoon or early evening samples which shall serve as a biological validation of our assay for wild female bonobos.

Rank-effects on outcome variables are often the result of contest competition (Janson 2000). With dominance rank varies the exposure to physical and psychosocial stressors (Sapolsky 2005) on the one hand and access to resources on the other (Deag 1977). We have previously shown for the females in this study that high-ranking females move less within food patches and have higher intake rates than low-ranking females independent of food resource characteristics (Nurmi et al. 2018), indicating that dominants enjoy some degree of priority of access to food resources. Against this background, the correlation between dominance rank and cortisol levels could be positive, if dominants lack support and are challenged by subordinates, or negative if subordinates are frequently exposed to dominance behaviors from high-ranking females (Goymann and Wingfield 2004). Our results of increased cortisol levels in dominants is in agreement with these predictions from the allostatic load framework because neither dominants nor subordinates support each other in polyadic conflicts against females (Nurmi et al. 2018). Moreover, subordinates may more easily avoid aggression from dominants in fission-fusion societies, and the rate of aggression towards subordinates is low compared to congeneric chimpanzee females (Nurmi et al. 2018). In more cohesive cercopithecines, where subordinates cannot avoid aggression, and where dominants enjoy matrilineal support, 
cortisol either decreases with decreasing rank or is not clearly associated with status (Weingrill et al. 2004). In contrast to female chimpanzees (Emery Thompson et al. 2010), this dominance effect was not moderated by female reproductive state.

With changing female reproductive state, energetic demands change (baboon, Papio cynocephalus: Muruthi et al. 1991; white-faced capuchin, Cebus capuchinus: McCabe and Fedigan 2007; American badger, Taxidea taxus: Harlow et al. 1985; little brown bat, Myotis lucifugus: Kurta et al. 1989; ungulates: Parker et al. 2009). Because cortisol is involved in energy allocation, a chronic shift of cortisol secretion is expected during pregnancy for mobilizing energy in support of fetal growth. In mammals, the physiological investment continues postpartum, with lactation generally considered as the most energetically demanding reproductive phase for a female (Gittleman and Thompson 1988, Clutton-Brock et al. 1989, Hinde et al. 2015). Primates tend to have relatively long gestation periods and produce dilute milk (Hinde and Milligan 2011), reflective of a life history strategy with costs of reproductive effort spread over a longer time due to shifting resource availability (Charnov and Berrigan 1993, Aiello and Key 2002). The exact energetic expenses of lactation stages can be hard to determine because milk composition and hence the maternal energy output might vary (bottlenose dolphin, Tursiops truncatus: West et al. 2007; house mouse, Mus musculus: König et al. 1988) or mothers may use behavioral compensation to minimise extra energy requirements (Murray et al. 2009; Altmann and Samuels 1992).

We found that females in the first six month of lactation had significantly higher urinary cortisol levels and this effect was independent of the dominance rank of the female. Our results are similar to findings from old world monkeys e.g. the first six months of lactation were associated with increased fecal glucocorticoid levels in blue monkeys (Cercopithecus mitis; Foerster et al. 2012). In two different populations of spotted hyenas (Crocuta crocuta), fecal cortisol levels were higher in lactating than non-lactating females (Goymann et al. 2001). Higher fecal glucorticoid levels in lactating compared to nonlactating females have also been reported in captive orangutans (Weingrill et al. 2011). Lactating female rhesus monkeys (Macaca mulatta) were found to have higher plasma cortisol levels than cycling females (Hoffman et al. 2010). Plasma cortisol levels follow a seasonal reproductive pattern in female degus (Octodon degus) and chipmunk (Tamias amoenus), with a distinct peak in cortisol levels during the lactation period (Kenagy et al. 1999, Kenagy and Place 2000). In several other studies, fecal glucocorticoid levels did not 
vary significantly with lactation state (Geoffroy's spider monkeys, Ateles geoffroyi: Rodrigues et al. 2015; chacma baboons, Papio ursinus: Weingrill et al. 2004; ring-tailed lemurs, Lemur catta: Cavigelli et al. 2003). In semi-free ranging mandrills (Mandrillus sphinx), there was no significant difference in glucocorticoid levels between cycling and lactating females (Setchell et al. 2008). Early lactation is associated with depressed energy balance in humans and chimpanzees (Ellison and Valeggia 2003, Emery Thompson et al. 2012). In contrast, we did not find that energy balance varied with reproductive state in bonobos. Besides the allostatic load of lactation in terms of energetic demands, especially in seasonally reproducing species lactation may be associated with low food availability and nutritional stress or psychological stressors may increase female cortisol levels due to infant vulnerability to threats ranging from increased attention from conspecifics and infanticide to predation (Fürtbauer et al. 2014).

Cortisol and insulin interact to affect lipid and protein metabolism. When access to food resources is restricted, energy intake is reduced and blood sugar levels drop, which directly affects cortisol secretion, which mobilizes energy from stored lipids and proteins instead of glucose oxidation (Dallman et al. 1993). In our study, we did not find an association between urinary C-peptide of insulin levels and urinary cortisol levels. The lack of correlation might reflect differences in excretion rates of the two hormones that would not be captured from the same urine sample. The morning values of urinary C-peptide reflect fasting values of insulin secretion while the high morning cortisol values reflect the endogenously driven diurnal peak. Our results bear resemblance to the pattern found in chimpanzees by Wessling et al. (2018a), who also did not find an effect of urinary C-peptide on urinary cortisol levels. However, as a minor methodological difference, our C-peptide and cortisol levels were corrected for creatinine, whereas Wessling et al. (2018a) corrected both physiological markers for specific gravity. Thus, the variation in cortisol levels was not mainly driven by nutritional stress. In other biological matrices, the total amount of cortisol correlated positively with the total amount of C-peptide in the amniotic fluid of pregnant women (Pschera et al. 1986), whereas a significant negative correlation was found between blood levels of insulin and cortisol in relation to the growth trajectory of malnourished children (Lunn et al. 1979).

One possibility is that the elevated cortisol levels of high-ranking female bonobos reflect indirect consequences of male-male mating competition. Mating in bonobos is characterised by polyandry and polygyny, and females exhibit highly variable genital 
swellings that are poor indicators of the timing of ovulation (Furuichi 2011, Douglas et al. 2016). Male bonobos form a stable linear dominance hierarchy, indicative of contest competition for mating partners (Surbeck et al. 2011). Males tend to range with theirs mothers, who provide agonistic support for their sons in conflicts over access to females (Furuichi and Ihobe 1994, Surbeck et al. 2011). While the pattern of reduced predictability of ovulation is predicted to decrease the level of reproductive skew in bonobos, high-ranking males have been shown to be over-represented in gaining reproductive success (Surbeck et al. 2017). Hence, there is strong indication that females can gain indirect fitness benefits by promoting the reproductive success of sons. Older females provide unidirectional, rather than reciprocal, agonistic support to younger females in conflicts against males (Tokuyama et al. 2016). By exclusion, the effects of dominance rank on cortisol levels are not likely to be due energetic challenges because dominance rank did not explain variation in energy balance (Nurmi et al. 2018) and female cortisol levels did not vary with energy balance in this study. Moreover, female energy balance did not vary with reproductive state, indicating females were not energetically challenged because of their reproductive state. High-ranking females tend to have a central role in the community (Furuichi and Ihobe 1994, Tokuyama and Furuichi 2017), and are therefore often involved in male-male conflicts by providing agonistic support for a son (those high-ranking females with sons) or to female-male conflicts by providing agonistic support for younger females against males. Moreover, these social challenges are likely to involve psychosocial unpredictability in part due to the femalefemale coalitions forming on an opportunistic basis, rather than based on affiliative relationships (Tokuyama and Furuichi 2016, Moscovice et al. 2017). In sum, the costs of sociality to high-ranked females might then derive mainly from such social stressors, albeit this still needs to be tested for.

Intersexual conflict has an important role in shaping social systems, and this conflict can be mediated by inter- and intrasexual relatedness (Rankin 2011). Therefore, considering the social strategies within and between the sexes yields a more complete picture of cooperation and competition over access to food and safety, and to mating partners, than considering social strategies within sexes separately. 


\section{ACKNOWLEDGEMENTS}

This study was funded by the Deutsche Forschungsgesellsschaft (DFG grants SCHU 1554/4-1 and HO 1151/9-1). We thank the Institut Congolaise pour la Conservation de la Nature (ICCN) for granting the research permit and the export permit for the urine samples. We thank the people of Lompole village in granting their permission to use their forest for research purposes. Lucas Goldstone, Josephine Hubbard and Lieven DeVreese provided invaluable assistance in the field. We are grateful to Ruth Sonneweber for help with the statistical analysis, and we thank Verena Behring for valuable advice with the analysis. We thank Roisin Murtaugh and Vera Schmeling for endocrinology laboratory analyses. 
Chapter 2 


\section{GENERAL DISCUSSION}

\section{Summary of results}

Social interactions among conspecific individuals give rise to competition and cooperation in various forms (Alexander 1974). Hence, the consideration of both costs and benefits of group living is of fundamental importance in socio-ecology. Compared to male-male competition, female-female competition has been, until recently, under-studied because females were thought to derive limited benefits from competition with one another (Stockley and BroJørgensen 2011). This view stemmed in part from observations of greater variance in male reproductive success compared to females (Bateman 1948). As data from long-term studies has accumulated, it has become clear that there can be considerable variation in the reproductive capacity of females (e.g. Pusey et al. 1997, Robinson et al. 2017), and the extent of reproductive skew can even be greater in females compared to males (Clutton-Brock and Huckard 2013). However, the sources of this variation are not always clear, as there are a number of different ways females can increase their reproductive success. Because of the high energetic costs of gestation and lactation (particularly in mammals), social strategies that help a female to secure a greater share of limiting food resources is expected to yield a selective advantage. Several studies have demonstrated a positive relationship between dominance status and reproductive success in females, with high-ranking females breeding earlier and more frequently, having faster growing offspring, and/or having offspring that are more likely to survive past the first year of life, compared to low-ranking females (CluttonBrock ad Huchard 2013). A correlation between female dominance status and reproductive success of females is likely to exert strong selection for attaining high dominance rank by females (Clutton-Brock and Huchard 2013). Female competitive strategies can also be selected to promote the reproductive success of their offspring, and it is important to bear in mind that female and male strategies can interact to influence female reproductive success. Innovations in the field of endocrinology have offered an unprecedented opportunity to illuminate the physiological causes and consequences of repeated social interactions among individuals.

In this thesis, I assessed the extent and form of female-female feeding competition in bonobos at Luikotale. My particular focus was to test for potential effects of contest competition, because this form of competition is predicted to cause dominance-rank dependent variation in energy balance, with implications for individual female reproductive 
success. Bonobos feed primarily on ripe fruits which may be a contestable resource and hence the potential for contest competition could be elevated according to predictions of the socio-ecological model, even though fission-fusion dynamics may mitigate contest effects. The socio-ecological framework places bonobos in the dispersal-egalitarian category, due to the female dispersal pattern and the weak female dominance relationships, yet this would not be in accordance with the assumption of relying mainly on contestable foods. Hence, the predictions of the socio-ecological model for bonobos necessitate proper testing, as there seem to be some elements of bonobo characteristics that may run counter to the assumptions or predicted outcomes of socio-ecological theory. Testing for the Resource Abundance hypothesis offers another piece of the puzzle. The hypothesis proposes that high quality terrestrial herbaceous vegetation reduces within-group scramble competition between fruit patches, whereas feeding from large fruit tree patches would reduce within-group contest competition. The integrative approach of my study allowed me to examine the links between ecological, social and physiological factors, and the relative strength of their effects on female energy balance and glucocorticoid levels. In combination with data on female-female coalitions, I was able to characterise the proximate mechanisms of feeding competition and to draw conclusions on the adaptive significance of female-female social interactions in a species known for its social tolerance. The results of my study enhance our understanding of bonobo feeding behaviours, how female bonobos can benefit from increased gregariousness and what are the costs involved, and whether these costs are equally shared among female.

I found that female bonobos form coalitions mostly with other females against males in line with previous research, and that despite contest competition induced dominance-rank effects on food intake and feeding efficiency, the energy balance of females did not vary with dominance rank but rather with monthly fruit availability. Females that occupy the highest rank in the group have increased glucocorticoid levels compared to all other females, and glucocorticoid levels are increased in females during the early lactation phase independent of the effect of rank. In the following, I will summarise briefly my main findings and discuss their implications in the socio-ecological framework. I discuss the potential mechanisms behind divergent behavioural and physiological indicators of contest competition. I also discuss the significance of the finding of female-female coalitions and the associated costs and benefits. I discuss the pros and cons of comparing energy balance and glucocorticoid levels, and offer possible mechanisms to explain the results on glucocorticoid levels. I conclude with a discussion on the differences between the Pan species, the modulating effect 
of male strategies and the implications of my study on the evolution of human social behaviour, along with an outlook for future directions.

\section{Mode of feeding competition in female bonobos}

An important assumption for feeding competition to occur is that food patches are depletable (Chapman et al. 1988). I began Chapter 1 by examining whether bonobos depleted food patches. I found that as the time the bonobos spent in the food patches increased, food intake rates decreased steadily (Figure 2b in Chapter 1; Nurmi et al. 2018) with a concurrent increase in the probability of movement (Figure 3b in Chapter 1; Nurmi et al. 2018). I was thus able to establish that food patches used by bonobos depleted over time. This is in line with observations on another primarily frugivorous species with a high degree of fission-fusion dynamics, the spider monkey (Ateles geoffroyi; Chapman 1988). The result of patch depletion lends support for the Cooperative Defense and Priority of Access hypotheses, but run counter to what is predicted for the Resource Abundance hypothesis. It is noteworthy however, that I was only able to consider patch depletion of those food types that I was able to include as focal tree patches, and I did not include terrestrial herbaceous vegetation patches as focal trees due to the difficulty of assessing patch size.

Measuring within-group feeding competition and separating the relative contributions of scramble and contest competition requires quantitative data on net energy gain of individuals in the group (Janson and van Schaik 1988). It has been shown that food intake rates have a strong impact on energy intake in primates (Schülke et al. 2006). I examined the form of feeding competition by testing the effect of food patch characteristics, feeding group size, and dominance rank on female food intake rates. I found that across all food patches, higher ranking females ingested more food per unit time spent in the patch compared to lower ranking females, albeit the effect was quite moderate (Figure $2 a$ in Chapter 1; Nurmi et al. 2018 ). Contest competition did not generally increase in patches that contained less food, were smaller, or contained an increasing number of co-feeding individuals. Using the same approach, I also tested for effects on female feeding efficiency, with probability of movement during feeding as a proxy. I found a clear effect of dominance rank on feeding efficiency, whereby low-ranking females were more likely to move in food patches than high-ranking females (Figure 3a in Chapter 1; Nurmi et al. 2018). Similar to the

food intake result, patches containing less food, smaller patches, or increasing party size did not induce stronger contest effects. Taken together, my results on feeding behaviour indicate 
that there is clear, yet moderate contest competition for food in bonobos with high-ranking females having higher food intake and reduced feeding effort during co-feeding, in support of the Cooperative defense hypothesis. My findings run counter to the hypothesis that bonobos feed from fruit trees too large to be depleted, because bonobos depleted fruit patches independent of patch size, leading to competition. One possible explanation for these results is that low-ranking females avoid conflict with high-ranking individuals by settling on a feeding spot containing less (Houle et al. 2007) or lower quality food (Houle et al. 2014), as found in chimpanzees (Pan troglodytes; Kahlenberg 2006, Houle et al. 2010), mountain gorillas (Gorilla beringei beringei; Wright et al. 2014), and possibly in Hanuman langurs (Semnopithecus entellus; Koenig and Borries 2001). Similar results of a positive effect of rank on food intake have been recorded in other female mammals, including red deer (Cervus elaphus: Thouless 1990), Appenine chamois (Rupicapra pyrenaica ornate; Lovari and Rosto 1985, Locati and Lovari 1991), vervet monkeys (Cercopithecus aethiops; Whitten 1983), white-faced capuchin monkeys (Cebus capucinus; Vogel 2005), olive baboons (Papio anubis; Barton and Whitten 1993) and mountain gorillas (Gorilla beringei beringei; Wright et al. 2014). In contrast, there was no effect of rank on food intake in mountain goats (Oreamnos americanus; Fournier and Festa-Blanchet 1995) or Assamese macaques (Macaca assamensis; Heesen et al. 2013).

Although food intake rate can have an important effect on net energy intake, it might not always match the actualised energy intake (Koenig 2002). Energy balance is the net difference between energy intake and energy expenditure (Sherry and Ellison 2007) and hence energy balance reflects competition on the ultimate level better than either energy intake or expenditure separately (Koenig 2002). Hormone analysis from non-invasively collected samples provides an important toolkit for supplementing studies on social behaviours. Insulin is a hormone with a central role in mediating glucose homeostasis and promoting energy allocation to storage, growth and reproduction (Ellison 2017). Changes in insulin levels can be studied in field conditions based on measurements of urinary C-peptide, which has been validated as a biomarker of energy balance in many primate species, including bonobos (Descher et al. 2008, Surbeck et al. 2015). Thus, I used urinary C-peptide levels to examine whether the dominance rank effect on food intake and feeding efficiency translated into physiological consequences of feeding competition for female bonobos. I additionally tested other factors influencing energy balance, including travelling effort, mean party size and monthly proportion of fruit in diet (full list of variables: Table 5 in Chapter 1; Nurmi et al. 2018). I did not find an effect of dominance rank on female energy balance, in 
support for the prediction of the Resource Abundance hypothesis. My result is congruent with findings from most other studies on female primates, which did not find a relationship between urinary C-peptide levels and dominance rank (baboons, Lodge 2012; capuchins, Bergstrom 2015; Sanje mangabeys, McCabe and Emery Thompson 2015; mountain gorillas at Virunga Volcanoes, Grueter et al. 2014). A positive relationship between dominance rank and energy balance (based on calculated energy intake/expenditure) was found in female mountain gorillas at Bwindi (Wright et al. 2014). In contrast, studies on male primates have reported both negative and positive relationships between urinary C-peptide levels and dominance rank (e.g. rhesus macaques, Higham et al. 2011; chimpanzees, Emery Thompson et al. 2009; bonobos, Surbeck et al. 2015). Nevertheless, I did find that monthly proportion of fleshy fruits in the diet had a significant positive effect on female C-peptide levels (Table 5 and Figure 4 in Chapter 1; Nurmi et al. 2018), in support of the Resource Abundance hypothesis. I was also able to establish that the average morning C-peptide levels were elevated following days when bonobos fed on Dialium spp. fruits (containing high sugar content; Beaune et al. 2013a), compared to the morning levels of the same individuals following days of dry seed consumption (Figure $1 \mathrm{a} / \mathrm{b}$ in Chapter 1 ; Nurmi et al. 2018). My results are in agreement with findings from the chimpanzee community at Taï, Ivory Coast, where the urinary C-peptide levels of both females and males correlated positively with ripe fruit availability (Wessling et al. 2018b). In other primate studies, feeding on preferred foods has a positive relationship with female urinary C-peptide levels in whitefaced capuchins (Bergstrom 2015), mountain gorillas (Grueter et al. 2014), and Kanyawara chimpanzees (Emery Thompson et al. 2014). In contrast to my findings, ripe fruit availability did not explain much of the variation in urinary C-peptide levels of female and male chimpanzees at Fongoli (Wessling et al. 2018a). Instead, energy balance of Fongoli chimpanzees showed variation with overall food availability, highlighting the importance of non-ripe fruit items in their diet (Wessling et al. 2018a).

\section{Effects of dominance rank on feeding efficiency but not on energy balance}

My combined findings indicate that higher-ranking females do not have better energy balance than lower-ranking females, despite having better access to food. The lack of dominance rank-related benefit for energy balance may be due to a variety of reasons. Urinary C-peptide levels may reflect other metabolic outcomes besides energy intake and expenditure from travel. In addition, costs of basal metabolic rate, reproductive state or illness are reflected (Ellison and Valeggia 2003, Emery Thomson et al. 2009, McCabe et al. 
2013). In the energy balance model, I controlled for reproductive state by indicating whether the female was lactating or not, whereas I did not include health status of the female due to the low number of individuals showing external symptoms of illness and lack of physiological data on health status. With regard to reproductive state, it is possible that high-ranking females were indeed able to obtain energetic gains through contest competition, but converted these gains to benefits for their offspring, either by producing more or better quality milk. This energetic investment in offspring would be expected to manifest as shorter inter-birth intervals or better offspring survival, rather than better energy balance. It is currently not known whether high-ranking bonobos have shorter inter-birth-intervals or high offspring survival compared to low-ranking females. In chimpanzees, high-ranking females have shorter inter-birth intervals and higher infant survival, including faster maturing female offspring (Pusey et al. 1997).

Even though high-ranking females have higher intake rates than low-ranking ones, the difference was very moderate, and hence may not have been strong enough to yield measurable differences in energy balance. It is also possible, that lower-ranking females may be able to compensate for lower intake rates by arriving at food resources before higherranking females, and spending longer time feeding before needing to move to a less profitable position in the food patch, which would then yield similar energy balance across females. Low-ranking females have been found to spend more time feeding compared to high-ranking females in blue monkeys (Cercopithecus mitis stuhlmanni; Pazol and Cords 2005) and in chimpanzees (Pan troglodytes; Murray et al. 2006), whereas dominance rank did not affect time spent feeding in mountain gorillas (Gorilla beringei beringei; Wright et al. 2014). However, in bonobos, old females are more likely to initiate group movements than young females or males (Tokuyama et al. 2017), and hence it may be more probable that young females follow older females to food patches so that low-rankers would enter at the same time or after high-rankers (under the assumption that dominance rank increases with age in bonobos). Alternatively, low-ranking females might increase their feeding time by staying food patches after high-rankers have departed. Based on my personal observation, low-ranking females do occasionally remain in feeding trees after most individuals departed, especially when the other community members remain near the feeding patch to rest, or prepare for night nesting. The effect of dominance rank on feeding would still need to be tested for, especially because increasing feeding time in order to compensate for lower energy intake may trade-off against other fitness enhacing activities, such having less time for resting (Pazol and Cords 2005) or affiliate behaviours. Subordinate females might opt out 
from the main party to feed alone in another fruit tree patch to compensate for their lower food intake, which can also increase predation risk (Vogel and Janson 2011). I therefore tested, how often females were missing from party scans and whether the average party size a female travelled in, correlated with dominance rank. I found that dominance rank did not predict how often a female was missing from the main party, nor did it correlate with the average party size of a female (Figure $5 a$ in Chapter 1; Nurmi et al. 2018). If high- and lowranking females are found in parties of similar average size, there is still the possibility that females assort according to dominance rank such that sub-parties are composed of similarly ranked females. However, it has been shown that dominance rank does not predict female association patterns following fissions, which speaks against the notion that females assort by rank in parties (Moscovice et al. 2017). I therefore concluded that lower-ranking females do not seem to compensate for lower food intake and increased feeding effort by splitting off from the main party to feed further.

Subordinate females may also compensate their lower energy gain in fruit tree patches by feeding more on terrestrial herbaceous vegetation, when parties travel between fruit patches (feed-as-you-go hypothesis sensu Wragham et al. 1996). The feed-as-you-go hypothesis suggests that bonobos travel at a lower velocity than chimpanzees between fruit patches, but this already implies that both low- and high-ranking individuals would have a similar opportunity to feed on terrestrial herbaceous vegetation. I compared the proportion of scans with feeding on terrestrial herbaceous vegetation and dominance rank, and did not find an association between the two factors. I therefore concluded that it is unlikely that lowranking females compensate for feeding deficits in fruit patches by consuming more terrestrial herbaceous vegetation. However, compared to scan samples, individual focal follows would yield a better insight in to potential compensatory feeding mechanisms. Moreover, fruits tend to have more energy than terrestrial herbs and I found elevated energy balance corresponding to months of high fruit feeding, future studies should examine diet quality.

\section{Female-female coalitions in bonobos}

Female-female coalitions among maternal relatives have a central role in primate socioecological models (Wrangham 1980, van Schaik 1989, Sterck et al. 1997). Coalitions refer to two or more individuals directing their behaviour together against a common third party target in a competitive or aggressive context (Bissonette et al. 2015). Research on cooperation in primates has tended to focus on inclusive fitness benefits derived from biasing cooperative 
behaviours toward genetic relatives (Langergraber 2012). However, the direct benefits of supporting maternal relatives may have been underestimated (Silk 2009) and coalitionary support for maternal relatives may help to reinforce the matrilineal dominance hierarchy, thus strenghtening the helpers own rank position (Langergraber 2012). Another neglected aspect is nepotism among paternal relatives, with more studies needed especially on male philopatric species where reproductive skew is biased towards few males (Schülke and Ostner 2008).

Despite the evidence for nepotistic biases contributing to the evolution of cooperative behaviours in primates (Silk 2009), cooperation among non-related individuals can also evolve by mutualism or reciprocity (Trivers 1971, Clutton-Brock 2009). Female bonobos form coalitions with other females under captive (Parish 1994, Stevens et al. 2006) and wild conditions (Tokuyama et al. 2016). The Cooperative Defence and Priority of Access hypotheses are difficult to distinguish based on my results on food intake, feeding effort and energy balance, because both hypotheses make similar predictions in terms of the outcomes, with variation only in the intensity of the effect (Table 1 in Chapter 1; Nurmi et al. 2018). However, the predictions concerning the targets of coalition are different between the two hypotheses; the Cooperative Defence hypothesis predicts coalitions to target mostly males, and the Priority of Access hypothesis predicts targets are mostly other females. The third hypothesis, the Resource Abundance hypothesis, does not predict the formation of femalefemale coalitions (although see page 70 in Wrangham 1993). By assessing data on agonistic interactions, I was able to show that during polyadic conflicts in a feeding context, most female-female coalitions targeted males (90\%). I also showed that in dyadic conflicts in the same context, females did not aggress males more often than other females. My findings lend support for the Cooperative Defence hypothesis. This hypothesis predicts that femalefemale coalitions should mainly target males, because males may dominate females in dyadic interactions, yet females may reduce the costs of aggression and gain feeding priority (over males) when food resources are economically defendable by forming coalitions with other females (White and Wood 2007). My results corroborate findings from bonobos at Wamba, where all female-female coalitions targeted males (Tokuyama et al. 2016). This also begs the question, whether female chimpanzees form coalitions with other females against males, and if not, what migth constrain female-female coalitions in chimpanzees? Sexual coercion by male chimpanzees imposes costs on females, as shown by elevations in cortisol levels of females during reproductive phases (cycling, parous) that attracted most male aggression (Muller et al. 2007). In the chimpanzees of the Kanyawa community at Kibale, 
female-female coalitions in chimpanzees have been found to target immigrant females (Kahlenberg et al. 2008b), whereas female-female coalitions targeting males are reported for the Sonso community, Budongo (Newton-Fisher 2006). A possible explanation for these discrepancies is that the Kanyawara female chimpanzees compete for core-areas in highquality neighborhoods that yield fitness benefits (Emery Thompson 2007) and hence it may be beneficial to cooperate in blocking incoming females, whereas Sonso females are described as more gregarious, with access to relatively abundant foods (Newton-Fisher 2003). However, resident Sonso females also show hostility towards female immigrants, suggesting resource competition for foods (Townsend et al. 2007). Spider monkeys ( $A$. geoffroyi) are frugivorous New World primates, which also exhibit fission-fusion dynamics and are male philopatric. Most female-female coalitions in spider monkeys targeted young immigrant females, suggestive of increased competition for food or breeding partners (Slater et al. 2009). This species shares traits with bonobos and chimpanzees, including male philopatry, frugivory and fission-fusion dynamics. Rimbach et al. (2014) found that spider monkeys ranged in smaller subgroups during high fruit availability, which is an atypical pattern under the assumption that fission-fusion dynamics alleviates feeding competition leading to the formation of smaller groups during low fruit abundance.

\section{Effects of dominance rank and reproductive state on allostatic load}

Besides measuring individual energy balance, the fitness consequences of social strategies can also be evaluated by assessing variation in glucocorticoid secretion (Higham 2016). As variation in glucocorticoid levels can be driven by energetic and social challenges, assessing glucocorticoid secretion provides a valuable complementary approach for understating the physiological consequences of feeding competition. Glucocorticoid secretion is an integral feature of the vertebrate stress response, enabling animals to cope with challenges posed by physical and psychological stressors (Romero 2004). The allostatic load concept provides a framework to study how dominance status is reflected in glucocorticoid levels, and helps in distinguishing between stressors deriving from the physical environment versus the social environment (Goymann and Wingfield 2004). The framework emphasizes, that the stress physiological profile of dominance status does not depend on being high- or low-ranked per se, but rather on the style of dominance rank maintenance and acquisition, along with the social milieu where it occurs (Sapolsky 1993). In Chapter 2, I applied urinary hormone analysis of cortisol (main glucocorticoid in primates) metabolite levels to assess whether the costs of gregariousness vary with dominance status in female bonobos. I found that the three 
female bonobos occupying the highest dominance rank stratum had elevated urinary cortisol levels compared to the 11 females on all other rank strata (Figure 2 and Figure 3a in Chapter 2). I did not find that the effect of binary dominance rank on cortisol levels changed with reproductive state. My finding of increased cortisol levels in dominant females is in accordance with the predictions of the allostatic load framework because high-ranking female bonobos do not generally have support from other females in conflicts with females, and lowranking females may be able to avoid aggression from high-ranking females due to the fission-fusion dynamics. High-ranking female bonobos tend to support low-ranking females unidirectionally in conflicts against males, indicating that coping mechanisms are available for subordinate females (Tokuyama et al. 2016). My results contrast with findings in chimpanzees to some extent because the effect of lactation on cortisol levels was stronger in low-ranking females compared to high-ranking females of the Kanyawara community (Emery Thompson et al. 2010). At Kanyawara, higher dominance rank was associated with occupying core areas containing better quality forage (Kahlenberg et al. 2008), and females in lower quality core areas were more peripheral with an increased risk of encountering males from neighbouring groups (Wilson and Wrangham 2003). However, Emery Thompson et al. (2010) found that (with all else being equal) cortisol levels of females with access to better quality foraging areas were higher than levels of more peripheral females. This hints at the possibility that although low-ranking female chimpanzees may suffer reduced foraging success, they might benefit by having less exposure to social stressors due to reduced association or aggression with other individuals. Another possibility is, that individual differences in glucocorticoid secretion do not result from dominance status characteristics directly, but rather from other traits that vary among individuals of different dominance status (Creel 2001). For example, Emery Thompson et al. (2010) noted that cortisol levels increased with age in the Kanyawara females. Age-related changes in the reactivity of the hypothalamopituitary-adrenal axis have been found in studies of humans (van Cauter et al. 1996) and rodents (Sapolsky et al. 1983), indicating that wear-and-tear due to life-long exposure to stressors may cause age-related loss of sensitivity to the inhibitory feed-back-loop of high circulating glucorticoid levels. In this respect, my finding of elevated cortisol levels in the highranking female bonobos might in part be explained by longer exposure to stressors overall, again under the assumption that dominance of female bonobos is positively correlated with age, as found in chimpanzees (Kahlenberg et al. 2008). Based on a visual inspection of the data points (Figure 2a in Chapter 2), the female who appears to be the oldest of the Bompusa community does not seem to have the highest mean cortisol levels. The ages of most females 
in the Bompusa bonobo community are not known and hence it was not possibly to properly test for a potential age effect on cortisol levels. In primates, most findings of higher glucocorticoid levels in high-ranking females have been reported in cooperative breeders (Creel 2001). Studies of cooperative breeders indicate that aspects of reproductive function and sex steroids (such as estrogens) are linked to the higher glucocorticoids levels of dominant females, as proposed for common marmosets (Callithrix jacchus; Saltzman et al. 1994), and African wild dogs (Lycaon pictus; Creel et al. 1997). In non-cooperatively breeding primates, dominance rank did not affect glucorticoid levels of females in most studies (reviewed by Beehner and Bergman 2017), including semi-free ranging mandrills (Mandrillus sphinx; Setchell et al. 2008), long-tailed macaques (Macaca fascicularis; van Schaik et al. 1991), and white-faced capuchins (Cebus capucinus; Carnegie et al. 2011).

Reproduction in mammals is an energetically demanding life history state for females (Gittleman and Thompson 1988). Reproductive status may therefore shape the endocrine response of the hypothalamo-pituitary-adrenal axis of females, as found in degus (Octodon degus; Kenagy et al. 1999), spotted hyenas (Crocuta crocuta; Goymann et al. 2001), rhesus macaques (Macaca mulatta; Hoffman et al. 2010), baboons (Papio cynocephalus; Gesquiere et al. 2008), and chimpanzees (Pan troglodytes schweinfurthii; Muller et al. 2007). I found that urinary cortisol levels of female bonobos were highest during early lactation (Figure $3 \mathrm{~b}$ in Chapter 2), corresponding to the first six months post-partum. The cortisol levels of females during later lactation (corresponding to six months post-partum to when the offspring is 2 years old) were slightly higher than the levels of cycling females (Figure $3 \mathrm{~b}$ in Chapter 2). My findings are in line with the notion that lactation is the most energetically demanding reproductive phase of female mammals (Gittleman and Thompson 1988, Clutton-Brock et al. 1989, Dufour and Sauther 2002). The first six months of lactation are associated with elevated faecal glucocorticoid levels in blue monkeys (Foerster et al. 2012). Elevated glucocorticoid levels during lactation compared to non-lactation have been found in spotted hyenas (Goymann et al. 2001), rhesus monkeys (Hoffman et al. 2010), and orangutans (Pongo spp.; Weingrill et al. 2011), whereas other studies have not found an effect of lactation on glucocorticoid levels (chacma baboons, Papio hamadryas ursinus, Weingrill et al. 2004; ring-tailed lemurs, Lemur catta, Cavigelli et al. 2003; white-faced capuchins, Carnegie et al. 2011; spider monkeys, Ateles geoffroyi, Rodrigues et al. 2015).

Elevated glucocorticoid levels may reflect food-related challenges, as found in seabirds (Kitaysky et al. 2010), African elephants (Loxodonta africana, Foley et al. 2001) and 
chimpanzees (Muller and Wrangham 2004). Comparing the relationship between levels of Cpeptide of insulin and glucocorticoids may help in disentangling the relative importance of nutritional status in affecting allostatic load, because both C-peptide and glucocorticoids are metabolic biomarkers, yet glucocorticoids are in addition important mediators of the stress response (Beehner and Bergman 2017). In my study, urinary C-peptide levels did not explain the variation in cortisol levels (Figure 4 in Chapter 2) suggesting, that cortisol excretion was not elevated due to nutritional status in female bonobos. In line with my findings, there was an absence of a correlation between urinary C-peptide and cortisol levels in chimpanzees at Fongoli and at Taï, where the former inhabit a savanna-mosaic habitat and the latter a lowland rainforest (Wessling et al. 2018b). In my study, I compared the two biomarkers in same urine samples collected before $10 \mathrm{AM}$. The morning values of urinary C-peptide reflect fasting values of insulin secretion, whereas the high morning cortisol values reflect an endogenously oscillating diurnal peak (Oster et al. 2017), which might partially obfuscate the relationship. A study on humans using experimentally elevated morning cortisol levels found that an increase in plasma cortisol was followed by a significant decrease in insulin secretion rates and plasma insulin levels within one hour, while plasma glucose levels did not start increasing until four hours after the hydrocortisone administration (Plat et al. 1996). The study suggested that elevated morning cortisol levels at physiological levels are likely to coincide with minimal insulin secretory responses. The study by Plat et al. (1996) also demonstrated the several hour delay in gluconeogenesis upon elevated cortisol levels, illustrating the differences in the time-course between cortisol and insulin actions. Lunn et al. (1979) found a negative correlation between plasma insulin and cortisol levels of malnourished childen in relation to their growth trajectories, whereby plasma cortisol had a negative and plasma insulin a positive correlation with both height and weight. One possibility for the clearer relationship between the two biomarkers in the study of Lunn et al. (1979) compared to my results is the difference in sampling timeline, whereby they sampled each individual at mothly intervals over three years, while I had overall fewer individuals sampled on shorter timescale and more frequently.

Early lactation is associated with depressed energy balance in chimpanzees (Emery Thompson et al. 2012) and humans (Ellison and Valeggia 2003), and therefore it is possible that the higher cortisol levels of females in early lactation state are due to nutritional stressors. Counter to this expectation, I did not find that early lactation was associated with much lower C-peptide levels compared to the levels of females during cycling or later lactation (Figure 5 in Chapter 2). Primates have several strategies for increasing energy intake to compensate 
for the costs of lactation, including reductions in physical activity, increasing metabolic efficiency, increasing caloric intake, or relying on stored body tissue (revied in Dufour and Sauther 2002). Female chimpanzees at Gombe have been shown to consume higher quality foods during lactation and pregnancy (Murray et al. 2009), whereas in Hanuman langurs, females in different reproductive phases did not consume foods with significantly different energetic values (Koenig et al. 1997). The daily energetic costs of lactation are generally though to be relatively low in large-bodied primates compared to other mammals of similar body size (Dufour and Sauther 2002), due to the prolonged offspring growth period (Charnov and Berrigan 1993) and relatively dilute breast milk (Hinde et al. 2009). The possibility, that female bonobos use compensatory strategies, such as more selective feeding or longer feeding times during lactation, would still need to be tested for.

The combined results of my second study have shown that the top-ranking female bonobos have elevated cortisol levels compared to females on all other ranks, suggesting increased allostatic load in these high-ranking females. I have suggested that these rank effects on cortisol levels are unlikely to stem from nutritional challenges, because dominance rank did not affect energy balance (Chapter 1) and energy balance did not explain variation in cortisol levels. The rank-effect on cortisol levels did not depend on female reproductive state in my study. However, this possibility would preferably need to be investigated further with a much larger set of urine samples from females in different reproductive states, as I had a only a limited number of samples per female representing different reprodutice states. I showed that females had elevated cortisol levels especially during early lactation compared to females during cycling or later lactation. I further established that these differences in cortisol levels were unlikely to be driven by energetic challenges because energy balance did not vary with reproductive state. Using inference by exclusion, I concluded that the elevated allostatic load of top-ranking females is likely to derive from social stressors. These social stressors are possibly linked to the central role of high-ranking females in bonobo communitites, where they provide agonistic support to younger, unrelated females during conflicts with males (Tokuyama et al. 2016, Nurmi et al. 2018), and/or for their sons during male-male conflicts over mating opportunities (Surbeck et al. 2011). 


\section{Conclusions and future directions}

Taken together, my field study of feeding behaviour and endocrinology in wild female bonobos makes an important contribution to our understanding of primate socio-ecology. My first chapter has helped to fill an important gap concerning female-female feeding competition in a species, which is widely known for its social tolerance. By demonstrating that bonobos depleted food resources and that female bonobos experience contest competition for foods, my findings provide a base for future studies of this, and of other species, where resource competition does not involve overt aggression. This is particularly important, because several generations of hypotheses proposed for the higher female gregariousness in bonobos compared to the congeneric chimpanzees have been formulated under the assumption that feeding competition is reduced in bonobos. The most prominent hypothesis to explain the species differences within genus Pan is the Resource Abundance hypothesis. The most recent incarnation of the Resource Abundance hypothesis is the Self Domestication hypothesis (Wrangham and Pilbean 2001). The Self Domestication hypothesis also builds on the premise that bonobos experience reduced scramble and contest competition compared to chimpanzees, allowing female bonobos to travel in larger, more stable parties than those of most chimpanzee communities. These larger parties in turn facilitate female-female coalitions, which can thwart male coercion. The high costs of male aggression to female fitness is then expected to select for less aggressive (or increasingly tolerant) male bonobos, who primarily ally with their female relatives, leading to increases in fitness via selfdomestication (Hare et al. 2012, Hare et al. 2017). My combined findings of patch depletion and the positive effect of dominance rank on food intake run counter to the predictions of both the Resource Abundance and the Self Domestication hypothesis, because contest competition would not be expected to prevail over evolutionary time, when foods would occur in large patches supplemented by highly abundant terrestrial herbaceous vegetation. The argument of contest effects fading over evolutionary time would be especially valid if females would use costly, frequent physical aggression or chases to gain feeding priority. Although I was not able to analyse agonistic rates in feeding patches due to methodological limitations, I suggested that the effects of contest competition for food in bonobos are more likely due to avoidance by subordinate females of dominant females. Under such a scenario, the costs of contest competition for high-ranking females could be expected to be reduced. There is also a possibility that high-ranking females exert their dominance outside of the feeding context, which could consolidate their priority of access to foods during feeding bouts. Further research to investigate these scenarios may include analyses of agonistic rates in feeding 
and non-feeding contexts, spatial position of females in feeding patches, individual proximities in the patch, and patterns of entries and exits to and from the patches.

My combined results on female energy balance and cortisol levels add important empirical data on the physiological consequences of the daily social interactions among female bonobos. The socio-ecological model conventionally categorises female bonobo social relationships as dispersal-egalitarian (Sterck et al. 1997). This category reflects shallow dominance relationships among unrelated females, whereby aggression is expected to be less severe and bidirectional (Chapais and Berman 2004). The lack of dominance rank effect on female energy balance in bonobos is in agreement with the socio-ecological model in this respect. This also draws attention to considering factors that may mitigate feeding competition in bonobos, because low-ranking ranking females appear to be compensating for lower intake rates. The fission-fusion dynamics implies flexibility to cope with contest competition. Another coping mechanism, which needs to be investigated further, is the extent of dietary flexibility and foraging strategies in bonobos. Despite the importance of ripe fruits, bonobos also feed on tree foliage, terrestrial herbs, underground items such as truffels, just to name a few. Therefore, the relative importance of nutrient balancing in the bonobo diet, including assessing variation in individual dietary choices depending on sex, age, or reproductive status are potential avenues for future research. In my study, I did not include pregnant females in the glucocorticoid analysis. Future studies focusing on the reproductive energetics of female bonobos would yield more insight into how females deal with the energetic challenges of gestation and lactation. Pregnant female chimpanzees have been found to reduce their physical activity (Murray et al. 2009), and to be less social, particularly during times before parturition (Pusey et al. 2008). Based on my personal observations, particularly the younger female bonobos seemed to range alone weeks before parturition.

From a game-theoretical perspective, the slow life-history strategy of bonobos may also reflect means of mitigating escalated contest competition. One of the predictions of game theory posits, that opponents with closely matched resource holding power would show the greatest tendency for escalated conflicts, whereas in cases of disparity between the resource holding power of the opponents, the opponent with higher resource holding power is expected to win while the loser withdraws following conventional display (Parker 1974b). Giving birth to one offspring at a time, the slow growth of offspring, and the relatively small group size in bonobos has the consequence, that there may be fewer matched opponents at any time in the community, leading to fewer opportunities for escalated conflict in this species. However, 
a broader analysis would be needed to compare other species with similar life histories, to those species with larger cohorts of offspring. From an inclusive fitness theory perspective, it is possible that female-biased dispersal in bonobos is selected for reduced local competition for food among female relatives, as the benefits of altruism can be cancelled by increased competition between relatives when dispersal is limited (West et al. 2002), albeit it is not likely to be the main reason (Lee and Strier 2015). Johnstone and Cant (2010) have modelled the relatedness outcomes in social groups with female-biased dispersal, male philopatry and within-group mating. What they have shown is that local relatedness increases with female age, which in turn would favour late-life helping (Johnstone and Cant 2010). The asymmetries in relatedness between young and old females arise because young females join new groups where they mate with males who are related to the older females in the group, resulting in offspring, which are genetically similar to the old females (Koster et al. 2018). The asymmetry is thought to resolve reproductive conflicts to the advantage of younger females (Kostner et al. 2018), which might in turn help to explain why older female bonobos provide unidirectional agonistic support to younger females.

By showing, that the top-ranked female bonobos may face stronger social stressors, I have set an interesting stage for additional research on the proximate mechanisms that drive this pattern, and how consistent this rank-dependent glucocorticoid pattern is within the same, and in other bonobo communities over time. I have proposed that the centrality of older, high-ranking females in the bonobo community exposes them to male-male conflicts over mating partners. High-ranking females may therefore accumulate costs, manifested as elevated cortisol levels, by providing agonistic support to their sons, while these costs may be offset through the direct benefit of gaining dominance over other males, and by the indirect benefit of gaining grand-offspring sired by their son(s) (Surbeck et al. 2017). Similarly, highranking females may accumulate costs by providing unidirectional agonistic support to younger females, with the benefits of maintaining dominance over males and by attracting potential mating partners for their sons. What remains to be tested is, whether high-ranking females with sons are more likely to provide support for young females, compared to highranking females without sons.

My finding concerning female-female coalitions contributes towards existing literature on female social strategies in bonobos, corroborating the importance of flexible alliance formation among unrelated females. My finding of female-female coalitions targeting mostly males does not comply with the prediction of the Resource Abundance hypothesis. However, 
this depends on how strictly we interpret the Resource Abundance hypothesis, because it contains several generations of sub-hypotheses, with adjusted predictions. The assumption of large food patches and abundant, high quality terrestrial herbs could be predicted to do away with the need of forming female-female coalitions in defence of food resources. However, the later versions of the Resource Abundance hypothesis, such as the Self Domestication hypothesis, emphasize, that while food abundance may facilitative femalefemale cooperation against males, it is mainly selected by other factors than feeding competition with males, namely male coercion of females and male efforts to dominate females (Hare et al. 2012). In comparison, the Cooperative Defence hypothesis remains silent about the mechanisms facilitating female gregariousness in bonobos, while proposing that female-female cooperation is mainly selected by feeding competition with males. These contrasting factors raise an important point for future investigation. In particular, more data are needed to understand the relative importance of males as competitors for food on the one hand, and more detailed observations to quantify the relative risks of male coercion in bonobos on the other. One of the observational challenges during feeding bouts is the clear context-specific assignment of male-female agonistic interactions as either food-related or coercion-related.

From a broader perspective, the points raised about males tie in with the primate socio-ecological model, which incorporates the modulating effect of male coercion on female strategies, yet does not currently consider feeding competition by males as an important factor affecting female social relationships. The traditional notion of female reproductive effort being more constrained by access to food compared to males rests mainly on the low energetic investment needed by mammalian males to produce gametes (Trivers 1972). This view tends to overlook, that the energetic costs of male mating effort can be quite considerable (Emery Thompson and Georgiev 2014) and that variation in testosterone production depends on longer-term energetic condition (Ellison 2003).

A full understanding of female-female cooperation and competition in multi-male multi-female social groups necessitates the simultaneous consideration of male social strategies. This is particularly relevant for understanding the factors shaping the social system of bonobos, where females are able to dominate males via coalitionary support of other females, and increase their inclusive fitness via coalitionary support of their sons. Demography, patterns of relatedness, and nutritional ecology are additional key factors in understanding variation in social systems. My thesis has enhanced our understanding of the 
costs and benefits of sociality in female bonobos and highlighted promising directions for future research. My summary of the most pertinent avenues for future studies is as follows:

1) More detailed studies of foraging strategies, including data on male food intake rates to assess the relative importance of males as competitors for food. Individual focal data on females for a more detailed analysis of potential compensatory feeding mechanisms, along with a qualitative assessment of possible inter-individual variation in terrestrial herbaceous vegetation consumption. Nutritional analyses in combination with longitudinal feeding data and energy balance data to assess dietary flexibility in bonobos.

2) Supplementary data to investigate the proximate mechanisms of feeding competition among females, including data on spatial position of individuals, group spread and proximities in feeding patches. Assessing avoidance behaviours and feeding times with data on entries and exits to and from feeding patches, in combination with data on agonistic displacements.

3) Analyses of agonistic behaviour in feeding- and non-feeding contexts, focal data to compare agonistic behaviour of females without adult sons and females with adult sons, particularly during periods of male dominance rank instability.

4) Assessing the relative importance of agonistic interactions on female cortisol levels.

5) Social network analyses to quantify the degree of female centrality in bonobos.

6) Comparative studies of feeding competition and foraging ecology of bonobo communities inhabiting forest-savanna mosaic habitat (Narat et al. 2015) to better understand the relative importance of ecological factors in facilitating female gregariousness in bonobos.

7) Ultimately, assessing reproductive success of female bonobos with comparative data on age at first reproduction, inter-birth-intervals, probability of infant survival, and maternal investment in male versus female offspring, particularly in relation to female dominance status. 


\section{REFERENCES}

Abbot D.H. Abbott DH, Keverne EB, Bercovitch FB, Shively CA, Mendoza SP, Saltzman W, Snowdon CT, Ziegler TE, Banjevic M, Garland T, Sapolsky RM. Are subordinates always stressed? A comparative analysis of rank differences in cortisol levels among primates. Horm Behav. 43:67-82.

Ahnesjö I, Kvarnemo C, Merilaita S. 2001. Using potential reproductive rates to predict mating competition among individuals qualified to mate. Behav. Ecol. 12:397-401.

Aiello LC, Key C. 2002. Energetic consequences of being a Homo erectus female. Am J Hum Biol. 14:551-565.

Alatalo RV, Eriksson D, Gustafsson L, Larsson K. 1987. Exploitation competition influences the use of foraging sites by tits: Experimental evidence. Ecology. 68:284-290.

Alberts SC. 2012. Magnitude and sources of variation in male reproductive performance. In: Mitani JC, Call J, Kappeler PM, Palombit RA, Silk JB, editors. The Evolution of Primate Societies. Chicago and London: The University of Chicago Press. pp. 412-431.

Alexander RD 1974. The evolution of social behavior. Ann. Rev. Ecol. Syst. 5:325-383.

Altmann J. 1974. Observational study of behavior: sampling methods. Behaviour 49:227-267.

Altman J, Samuels, A. 1992. Costs of maternal care: Infant carrying in baboons. Behav Ecol Socbiol. 29(6):391-398.

Andersson M. 1994. Sexual Selection. In: Krebs JR, Clutton-Brock T, editors. Monographs in Behavior and Ecology. Princeton, New Jersey: Princeton University Press.

Anestis SF, Bribiescas RG. 2004. Rapid changes in chimpanzee (Pan troglodytes) urinary cortisol excretion. Horm Behav. 45:209- 213. 
Archie EA, Morrison TA, Foley CAH, Moss CJ, Alberts SC. 2006. Dominance rank relationships among wild female African elephants, Loxodonta africana. Anim Behav. 71:117127.

Asensio N, Korstjens AH, Schaffner CM, Aureli F. 2008. Intragroup aggression, fission-fusion dynamics and feeding competition in spider monkeys. Behavior. 145:983-1001.

Aureli F, Cords M, van Schaik CP. 2002. Conflict resolution following aggression in gregarious animals: a predictive framework. Anim Behav. 64:325-343.

Aureli F, Schaffner CM, Boesch C, Bearder SK., Call J, Chapman CA, Connor R, Di Fiore A, Dunbar RIM., Henzi SP, Holekamp K, Korstjens AH, Layton R, Lee P, Lehmann J, Manson JH, Ramos-Fernandez G, Strier KB, van Schaik CP. 2008. Fission-fusion dynamics: new research frameworks. Curr Anthropol. 49:627-654.

Baayen RH. 2008. Analyzing linguistic data: a practical introduction to statistics using R. Cambridge: Cambridge University Press.

Baayen RH. 2013. Multivariate Statistics. In: Podesva R, Sharman D. editors. Research Methods in Linguistics. Cambridge: Cambridge University Press. pp. 337-372.

Badrian AJ, Badrian NL. 1984. Social organization of Pan paniscus in the Lomako forest, Zaire. In: Sussman RL, editor. The Pygmy Chimpanzee: evolutionary biology and behavior. New York and London: Plenum Press. p. 325-346.

Badrian NL, Malenky RK. 1984. Feeding ecology of Pan paniscus in the Lomako forest, Zaire. In: Sussman RL, editor. The Pygmy Chimpanzee: evolutionary biology and behavior. New York and London: Plenum Press. p. 275-299.

Bahr NI, Palme R, Möhle U, Hodges JK, Heistermann M. 2000. Comparative aspects of the metabolism and excretion of cortisol in three individual nonhuman primates. Gen Comp Endocrinol. 117:427-438. 
Balsalobre A, Brown SA, Marcacci L, Tronche F, Kellendonk C, Reichardt HM, Schütz G, Schibler U. 2000. Resetting of circadian time in peripheral tissues by glucocorticoid signaling. Science. 289:2344-2347. DOI: 10.1126/science.289.5488.2344.

Barr DJ, Levy R, Scheepers C, Tily HJ. 2013. Random effects structure for confirmatory hypothesis testing: keep it maximal. J Mem Lang. 68:255-278.

Bartlett TQ, Sussman RW, Cheverud JM. 1993. Infant killing in primates: A review of observed cases with specific reference to the sexual selection hypothesis. Am Anthropol. 95:958-990.

Barton RA. 1993. Sociospatial mechanisms of feeding competition among female olive baboons Papio anubis. Anim Behav. 46:791-802

Barton RA, Whiten A. 1993. Feeding competition among female olive baboons, Papio anubis. Anim Behav. 46:777-789.

Barton RA, Byrne RW, White A. 1996. Ecology, feeding competition and social structure in baboons. Behav Ecol Sociobiol. 38:321-329.

Bateman AJ. 1948. Intra-sexual selection in Drosophila. Heredity. 2:349-368.

Bates D, Mächler M, Bolker BM, Walker SC. 2015. Fitting linear mixed-effects models using Ime4. J Stat Softw. 67(1):1-48. doi:10.18637/jss.v067.101.

Beaune D, Bretagnolle F, Bollache L, Hohmann G, Surbeck M, Bourson C, Fruth B. 2013 a. The bonobo-Dialium positive interactions: seed dispersal mutualism. Am J Primatol. 75:394403.

Beaune D, Bretagnolle F, Bollache L, Hohmann G, Surbeck M, Fruth B. 2013b. Seed dispersal strategies and the threat of defaunation in a Congo rainforest. Biodivers Conserv. 22:225-238.

Beehner JC, Bergman TJ. 2017. The next step for stress research in primates: To identify relationships between glucocorticoid secretion and fitness. Horm Behav. 91:68-83. 
Beehner JC, McCann C. 2008. Seasonal and altitudinal effects on glucocorticoid metabolites in a wild primate (Theropithecus gelada). Physiol Behav. 95:508-514.

Behringer V, Deschner T, Deimel C, Stevens JMG, Hohmann G. 2014. Age-related changes in urinary testosterone levels suggest differences in puberty onset and divergent life history strategies in bonobos and chimpanzees. Horm Behav. 66:525-533.

Belgardt BF, Stoffel M. 2014. SIK2 regulates insulin secretion. Nat Cell Biol. 16:210-212.

Berghänel A, Heistermann M, Schülke O, Ostner J. 2017. Prenatal stress accelerates offspring growth to compensate for reduced maternal investment across mammals. Proc Natl Acad Sci USA. 114(50):E10658-E10666.

Bergman TJ, Beehner JC. 2015. Measuring social complexity. Special issue: Social Evolution. Anim Behav. 103:203-209.

Bergstrom ML. 2015. Seasonal effects on the nutrition and energetic condition of female white-faced capuchin monkeys. Ph.D. Thesis, University of Calgary, Canada.

Bergouignan A, Habold C, Rudwill F, Gauquelin-Koch G, Normand S, Simon C, Blanc S. 2012. Urinary C-peptide is not an accurate bioindicator of energy balance in humans. Obesity. 20:683-688.

Bernstein IS. 1981. Dominance: The baby and the bathwater. Behav Brain Sci. 4:419-457.

Bernstein IS. 1988. Kinship and behavior in nonhuman primates. Behav Genet. 18:511-524.

Birch LC. 1957. The meanings of competition. Am Nat. 91(856):5-18.

Bissonnette A, Perry S, Barrett L, Mitani JC, Flinn M, Gavrilets S, de Waal FBM. 2015. Coalitions in theory and reality: a review of pertinent variables and processes. Behavior. 152:1-56.

Brockmann HJ, Barnard CJ. 1979. Kleptoparasitism in birds. Anim Behav. 27:487-514. 
Boesch C, Boesch-Achermann H. 2000. The Chimpanzees of the Taï Forest: Behavioural Ecology and Evolution. Oxford: Oxford University Press.

Boesch C, Hohmann G, Marchant LF. 2002. Behavioural diversity in chimpanzees and bonobos. Cambridge: Cambridge University Press.

Boesch C. 2009. The real chimpanzee: sex strategies in the forest. Cambridge: Cambridge University Press.

Bonser AM, Garcia-Webb P, Harrison LC. 1984. C-Peptide Measurement: Methods and Clinical Utility. Crit Rev Clin Lab Sci. 19(4):297-352. DOI: 10.3109/10408368409165766.

Boonstra R. 2013. Reality as the leading cause of stress: rethinking the impact of chronic stress in nature. Func Ecol. 27:11-23.

Borries C, Sommer V, Srivastava A. 1991. Dominance, age, and reproductive success in free-ranging female Hanuman langurs (Presbytis entellus). Int J Primatol. 12:231-257.

Borries C, Larney E, Lu A, Ossi K, Koenig A. 2008. Costs of group size: lower developmental and reproductive rates in larger groups of leaf monkeys. Behav Ecol. 19(6):1186-1194.

Bowen WD, Iverson SJ, Boness DJ, Oftedal OT. 2001. Foraging effort, food intake and lactation performance depend on maternal mass in a small phocid seal. Funct Ecol. 15:325334.

Boydston EE, Morelli TL, Holekamp KE. 2001. Sex differences in territorial behavior exhibited by the spotted hyena (Hyaenidae, Crocuta crocuta). Ethology 107:369-385.

Bradbury JW, Vehrencamp SL. 1976. Social organization and foraging in emballonurid bats. II. A model for the determination of group size. Behav Ecol Sociobiol. 1:383-404.

Bradbury JW, Vehrencamp SL. 1977. Social organization and foraging in emballonurid bats. III. Mating systems. Behav. Ecol. Sociobiol. 2:1-17. 
Brereton AR. 1995. Coercion-defense hypothesis: The evolution of primate sociality. Folia Primatol. 64:207-214.

Bronson FH. 1989. Mammalian reproductive biology. Chicago:University of Chicago Press. pp. 53-55.

Broom M, Koenig A, Borries C. 2009. Variation in dominance hierarchies among group-living animals: modeling stability and the likelihood of coalitions. Beha Ecol. 20:844-855.

Brown JL. 1964. The evolution of diversity in avian territorial systems. Wilson Bull. 76(2):160169.

Brown JL. 1969. Territorial behavior and population regulation in birds. A review and reevaluation. Wilson Bull. 81:293-329.

Brown JL, Orians GH. 1970. Spacing patterns in mobile animals. Annu Rev Ecol Syst. 1:239262.

Brown GR, Dickins TE, Sear R, Laland KN. 2011. Evolutionary accounts of human behavioural diversity. Phil Trans R Soc B. 366:313-324.

Bullinger AF, Burkart JM, Melis AP, Tomasello M. 2013. Bonobos, Pan paniscus, chimpanzees, Pan troglodytes, and marmosets, Callithrix jacchus, prefer to feed alone. Anim Behav. 85:51-60.

Burt WH. 1943. Territoriality and home range concepts as applied to mammals. J Mammal. 24:346-352.

Cain KE, Rosvall KA. 2014. Next steps for understanding the selective relevance of femalefemale competition. Front Ecol Evol. 2:1-3. DOI: 10.3389/fevo.2014.00032

Cameron AC, Trivedi PK. 1990. Regression-based tests for overdispersion in the Poisson model. J Econometrics 46:347-364. 
Cameron TC, Wearing HJ, Rohani P, Sait SM. 2007. Two-species asymmetric competition: effects of age structure on intra- and interspecific interactions. J Anim Ecol 76:83-93.

Cano-Huertes B, Rangel-Negrín A, Coyohua-Fuentes A, Chavira-Ramírez DR, CanalesEspinosa D, Dias PAD. 2017. Reproductive Energetics of Female Mantled Howlers (Alouatta palliata). Int J Primatol. 38:942-961.

Cant MA, Johnstone RA. 2008. Reproductive conflict and the separation of reproductive generations in humans. Proc Natl Acad Sci USA 105:5332-5336.

Carlson BA, Rothman JM, Mitani JC. 2013. Diurnal variation in nutrients and chimpanzee foraging behavior. Am J Primatol. 75:342-349.

Carnegie SD, Fedigan LM, Ziegler TE. 2011. Social and environmental factors affecting fecal glucocorticoids in wild, female white-faced capuchins (Cebus capucinus). Am J Primatol. 73:861-869.

Carpenter FL. 1987. Food abundance and territoriality: To defend or not to defend? Amer Zool. 27(2):387-399.

Cavigelli SA, Dubovick T, Levash W, Jolly A, Pitts A. 2003. Female dominance status and fecal corticoids in a cooperative breeder with low reproductive skew: ring-tailed lemurs (Lemur catta). Horm Behav. 43:166-179.

Chan S, Debono M. 2010. Replication of cortisol circadian rhythm: new advances in hydrocortisone replacement therapy. Ther Adv Endocrinol Metab. 1(3):129-138.

Chapais B. 1995. Alliances as a means of competition in primates: Evolutionary, developmental, and cognitive aspects. Yearb Phys Anthropol. 38:115-136.

Chapais B, Berman CM. 2004. Kinship and behavior in primates. Oxford: Oxford University Press. pp. 484.

Chapman CA. 1988. Patch use and patch depletion by the spider and howling monkeys of Santa Rosa national park, Costa Rica. Behaviour 105:99-116. 
Chapman CA, Chapman LJ, Wrangham R, Hunt K, Gebo D, Gardner L. 1992. Estimators of fruit abundance of tropical trees. Biotropica 24:527-531.

Chapman CA, White FJ, Wrangham RW. 1994. Party size in chimpanzees and bonobos: a reevaluation of theory based on two similarly forested sites. In: Wrangham RW, McGrew WC, de Waal FBM, Heltne PG, editors. Chimpanzee cultures. Cambridge: Harvard University press. pp. 41-57.

Charnov EL. 1976. Optimal foraging, the marginal value theorem. Theor Popul Biol. 9:129136.

Charnov EL, Berrigan D. 1993. Why do female primates have such long lifespans and so few babies? or Life in the slow lane. Evol Anthropol. 1:191-194.

Chase I, Bartolomeo C, Dugatkin LA. 1994. Aggressive interactions and inter-contest interval: how long do winners keep winning? Anim Behav. 48:393-400.

Cheney DL, Seyfarth RM. 1987. The influence of intergroup competition on the survival and reproduction of female vervet monkeys. Behav Ecol Sociobiol. 21:375-386.

Clay Z, de Waal FBM. 2014. Sex and strife: post-conflict sexual contacts in bonobos. Behaviour 152:313-334.

Clutton-Brock TH. 1974. Primate social organisation and ecology. Nature. 250:539-542.

Clutton-Brock TH. 2009. Cooperation between non-kin in animal societies. Nature. 462:5157.

Clutton-Brock TH, Albon SD, Guinness FE. 1989. Fitness costs of gestation and lactation in wild mammals. Nature. 337:260-262.

Clutton-Brock TH, Parker GA. 1992. Potential Reproductive Rates and the Operation of Sexual Selection. Q Rev Biol. 67:437-456.

Clutton-Brock T, Janson C. 2012. Primate socioecology at the crossroads: Past, present, and future. Evol Anthropol. 21:136-150. 
Clutton-Brock T, Huchard E. 2013. Social competition and its consequences in female mammals. J Zool. 289:151-171.

Collins DA, Busse CD, Goodall J. 1984. Infanticide in two populations of savanna baboons. In: Hausfater G, Hrdy SB, editors. Infanticide: Comparative and Evolutionary Perspectives. New York: Aldine Publishing. pp. 193-215.

Cords M. 2012. The behavior, ecology and social evolution of Cercopithecine monkeys. In: Mitani JC Call J, Kappeler PM, Palombit RA, Silk JB, editors. The evolution of primate societies. Chicago: The University of Chicago Press. pp. 91-112.

Craig JL, Douglas ME. 1986. Resource distribution, aggressive asymmetries and variable access

to resources in the nectar feeding bellbird. Behav Ecol Sociobiol. 18:231-240.

Creel S, Marusha Creel N, Mills MGL, Monfort SL. 1997. Rank and reproduction on cooperatively breeding African wild dogs: behavioral and endocrine correlates. Behav Ecol. 8:298-306.

Creel S, Dantzer B, Goymann W, Rubenstein DR. 2013. The ecology of stress: effects of the social environment. Funct Ecol. 27:66-80.

Cronin KA., De Groot E, Stevens JMG. 2015. Bonobos show limited social tolerance in a group setting: a comparison with chimpanzees and a test of the relational model. Folia Primatol. 86:164-177.

Crook JH, Gartlan JS. 1966. Evolution of primate societies. Nature. 210:1200-1203.

Czekala NM, Lance VA, Sutherland-Smith M. 1994. Diurnal urinary corticoid excretion in the human and gorilla. Am J Primatol. 34:29-34.

Dallman MF, Strack AM, Akana SF, Bradbury MJ, Hanson ES, Scribner KA, Smith M. 1993. Feast and famine: critical role of glucocorticoids with insulin in daily energy flow. Front Neuroendocrinol. 14:303-347. 
Dallman MF, Warne JP, Foster MT, Pecoraro NC. 2007. Glucocorticoids and insulin both modulate caloric intake through actions on the brain. J Physiol. 583.2:431-436.

Dantzer B, Westrick SE, van Kesteren F. 2016. Relationships between endocrine traits and life histories in wild animals: Insights, problems, and potential pitfalls. Integ Comp Biol. 56:185-197.

Darlington RB, Hayes AF. 2017. Power measurement error, and various miscellaneous topics. In: Little TD, editor. Regression analysis and linear models. New York: Guildford Publishing. pp. 519-548.

Davis DE. 1958. The role of density in aggressive behaviour of house mice. Anim Behav. 6:207-210.

Dawkins 1982. Selfish wasp or selfish strategy? The extended phenotype. Oxford: Oxford University Press. pp. 118-132.

Deag JM. 1977. Aggression and submission in monkey societies. Anim Behav. 25:465-474.

Debono M, Ghobadi C, Rostami-Hodjegan A, Huatan H, Campbell MJ, Newell-Price J, Darzy K, Merke DP, Wiebke A, Ross RJ. 2009. Modified-release hydrocortisone to provide circadian cortisol profiles. J Clin Endocrinol Metab. 94: 1548-1554.

De Jong G. 1976. A model of competition for food. I. Frequency-dependent viabilities. Am Nat. 110(976): 1013-1027.

de Manuel M, Kuhlwilm M, Frandsen P, Sousa VC, Desai T, Prado-Martinez J, HernandezRodriguez J, Dupanloup I, et. al. 2016. Chimpanzee genomic diversity reveals ancient admixture with bonobos. Science 354:477-481.

de Ruiter JR. 1986. The influence of group size on predator scanning and foraging behaviour of wedgecapped capuchin monkeys (Cebus olivaceus). Behavior. 98:240-258.

de Waal FBM, Yoshihara D. 1983. Reconciliation and redirected affection in rhesus monkeys. Behavior. 85:224-241. 
de Waal FBM. 1989. Behavioral contrasts between bonobo and chimpanzee. In: Marquardt P. editor. Understanding chimpanzees. Cambridge MA: Harvard University Press. pp. 154175.

de Waal FBM, Harcourt AH. 1992. Coalitions and alliances: a history of ethological research. In: Harcourt AH, de Waal FBM, editors. Coalitions and Alliances in Humans and Other Animals. Oxford: Oxford University Press. p. 1-19.

Denham WW. 1971. Energy relations and some basic properties of primate social organization. Am Anthropol. 73:77-95.

Deschner T, Heistermann M, Hodges K, Boesch C. 2003. Timing and probability of ovulation in relation to sex skin swelling in wild West African chimpanzees, Pan troglodytes verus. Anim. Behav. 66:551-560.

Deschner T, Kratzsch J, Hohmann G. 2008. Urinary C-peptide as a method for monitoring body mass changes in captive bonobos (Pan paniscus). Horm Behav. 54:620-626.

Dobson AJ. 2002. An Introduction to Generalized Linear Models. 2nd edition. London: Chapman \& Hall.

Dobson FS. 1982. Competition for mates and predominant male dispersal in mammals. Anim Behav. 30:1183-1192.

Douglas PH, Hohmann G, Murtagh R, Thiessen-Bock R, Deschner T. 2016. Mixed messages: wild female bonobos show high variability in the timing of ovulation in relation to sexual swelling patterns. BMC Evol Biol. 16:140.

Douglas PH, Ngonga Ngomo AC, Hohmann G. 2017. A novel approach for dominance assessment in gregarious species: ADAGIO. Anim Behav. 123:21-32.

Drews C. 1993. The Concept and Definition of Dominance in Animal Behaviour. Behavior 125:283-313. 
Dufour DL, Sauther ML. 2002. Comparative and evolutionary dimensions of the energetics of human pregnancy and lactation. Am J Hum Biol. 14:584-602.

Dugatkin LA. 1997. Winner and loser effects and the structure of dominance hierarchies. Behav Ecol. 8:583-587.

Dugatkin LA. 2001. Bystander effects and the structure of dominance hierarchies. Behav Ecol. 12:348-352.

Duijns S, Piersma T. 2014. Interference competition in a sexually dimorphic shorebird: prey behaviour explains intraspecific competition. Anim Behav. 92:195-201.

Dunbar RIM. 2002. Modelling primate behavioral ecology. Int J Primatol. 23:785-819.

Duthie L, Reynolds RM. 2013. Changes in the maternal hypothalamic-pituitary-adrenal axis in pregnancy and postpartum: Influences on maternal and fetal outcomes. Neuroendocrinology. 98:106-115.

Ellis L. 1995. Dominance and reproductive success among nonhuman animals: a crossspecies comparison. Ethol Sociobiol. 16:257-333.

Ellison PT. 2003. Energetics and reproductive effort. Am J Hum Biol. 15:342-351.

Ellison PT. 2017. Endocrinology, energetics, and human life history: A synthetic model. Horm Behav. 91:97-106.

Ellison PT, Valeggia CR. 2003. C-peptide levels and the duration of lactational amenorrhea. Fertil Steril. 80:1279-1280.

Emery Thompson M. 2013. Reproductive ecology of female chimpanzees. Am J Primatol. 75:222-237.

Emery Thompson M. 2017. Energetics of feeding, social behavior, and life history in nonhuman primates. Horm Behav. 91:84-96. 
Emery Thompson M, Kahlenberg SM, Gilby IC, Wrangham RW. 2007. Core area quality is associated with variance in reproductive success among female chimpanzees at Kibale National Park. Anim Behav. 73:501-512.

Emery Thompson M, Knott CD. 2008. Urinary C-peptide of insulin as a non-invasive marker of energy balance in wild orangutans. Horm Behav. 53:526-535.

Emery Thompson M, Muller MN, Wranhgam RW, Lwanga JS, Potts KB. 2009. Urinary Cpeptide tracks seasonal and individual variation in energy balance in wild chimpanzees. Horm Behav. 55:299-305.

Emery Thompson M, Muller MN, Kahlenberg SM, Wrangham RW. 2010. Dynamics of social and energetic stress in wild female chimpanzees. Horm Behav. 58:440-449.

Emery Thompson M, Muller MN, Wrangham RW. 2012. The energetics of lactation and the return to fecundity in wild chimpanzees. Behav Ecol. 23:1234-1241.

Emery Thompson M, Muller MN, Wrangham RW. 2014. Male chimpanzees compromise the foraging success of their mates in Kibale National Park, Uganda. Behav Ecol Sociobiol. 68:1973-1983.

Emlen ST. 1976. Lek organization and mating strategies in the bullfrog. Behav Ecol Sociobiol. $1: 283-313$.

Emlen ST, Oring LW. 1977. Ecology, sexual selection, and the evolution of mating systems. Science. 197:215-223.

Eriksson J, Siedel H, Lukas D, Kayser M, Erler A, Hashimoto C, Hohmann G, Boesch C, Vigilant L. 2006. Y-chromosome analysis confirms highly sex-biased dispersal and suggests a low male effective population size in bonobos (Pan paniscus). Molec Ecol. 15:939-949.

Field A. 2005. Discovering Statistics using SPPS. London: Sage Publications.

Finch CE, Rose MR. 1995. Hormones and the physiological architecture of life history evolution. Q Rev Biol. 70:1-52. 
Foerster S, Cords M, Monfort SL. 2012. Seasonal energetic stress in a tropical forest primate: Proximate causes and evolutionary implications. PLOS One. 7(11):e50108. doi:10.1371/journal.pone.0050108.

Foerster S, Franz M, Murray CM, Gilby IC, Feldblum JT, Walker KK, Pusey AE. 2016. Chimpanzee females queue but males compete for social status. Sci Rep. 6:35404; doi: 10.1038/srep35404.

Foley CAH, Papageorge S, Wasser SK. 2001. Noninvasive stress and reproductive measures of social and ecological pressures in free-ranging African elephants. Conserv Biol. 15(4): 1134-1142.

Forstmeier W, Schielzeth H. 2011. Cryptic multiple hypotheses testing in linear models: overestimated effect sizes and the winner's curse. Behav Ecol Sociobiol. 65:47-55.

Fournier F, Festa-Bianchet M. 1995. Social dominance in adult female mountain goats. Anim Behav. 49:1449-1459.

Fox J, Weisberg S. 2011. An R companion to applied regression. 2nd ed. Thousand Oaks: Sage Publications. URL: http://socserv.socsci.mcmaster.ca/jfox/Books/Companion

Freeberg TM, Dunbar RIM, Ord TJ. 2012. Social complexity as a proximate and ultimate factor in communicative complexity. Phil Trans R Soc B 367:1785-1801.

Fretwell SD. 1972. Populations in a Seasonal Environment. Princeton: Princeton University Press.

Fretwell SD, Lucas HL. 1970. On territorial behaviour and other factors influencing habitat distribution in birds. Acta Biotheor. 19:16-36.

Fruth B, Hohmann G. 2018. Food Sharing across borders. First observation of intercommunity meat sharing by bonobos at LuiKotale, DRC. Hum Nat. 29:91-103.

Furuichi T. 1989. Social interactions and the life history of female Pan paniscus in Wamba, Zaire. Int J of Primatol. 10:173-197. 
Furuichi T. 1997. Agonistic interactions and matrifocal dominance rank of wild bonobos (Pan paniscus) at Wamba. Int J Primatol. 18:855-875.

Furuichi T. 2011. Female Contributions to the Peaceful Nature of Bonobo Society. Evol Anthropol. 20:131-142.

Furuichi T, Ihobe H. 1994. Male bonding in non-human primates. Behavior. 130:211-228.

Furuichi T, Sanz C, Koops K. Sakamaki T, Ryu H, Tokuyama N, Morgan D. 2015. Why do wild bonobos not use tools like chimpanzees do? Behaviour 152:425-460.

Fürtbauer I, Heistermann M, Schülke O, Ostner J. 2014. Low female stress hormone levels are predicted by same- or opposite-sex sociality depending on season in wild Assamese macaques. Psychoneuroendocrinol. 48:19-28.

Gale JS, Eaves LJ. 1975. Logic of animal conflict. Nature. 254:463-464.

Ganslosser U, Dellert B. 1997. Experimental alterations of food distribution in two species of captive equids (Equus burchelli and E. hemionus kulan), Ethol Ecol Evol. 9(1):1-17. DOI: 10.1080/08927014.1997.9522898.

Gardner A, West SA, Wild G. 2011. The genetical theory of kin selection. J Evol Biol. 24:10201043.

Geist V. 1974. On the relationship of social evolution and ecology in Ungulates. Amer Zool. $14: 205-220$.

Georgiev AV, Emery Thompson M, Lokasola AL, Wrangham RW. 2011. Seed predation by bonobos (Pan paniscus) at Kokolopori, Democratic Republic of the Congo. Primates 52:309314.

Gerloff U, Hartung B, Fruth B, Hohmann G, Tautz D. 1999. Intracommunity relationships, dispersal pattern and paternity success in a wild living community of bonobos ( $P a n$ paniscus) determined from DNA analysis of faecal samples. Proc R Soc Lond. B266:1189-1195. 
Gesquiere LR, Khan M, Shek L., Wango TL., Wango EO, Alberts SA, Altmann J. 2008. Coping with a challenging environment: effects of seasonal variability and reproductive status on glucocorticoid concentrations of female baboons (Papio cynocephalus). Horm Behav. 54(3):410-416.

Girard-Buttoz C, Higham JP, Heistermann M, Wedegärtner S, Maestripieri D, Engelhardt A. 2011. Urinary C-peptide measurement as a marker of nutritional status in macaques. PLoS ONE 6(3): e18042.

Gittleman JL, Thomson SD. 1988. Energy allocation in mammalian reproduction. Amer Zool. 28:863-875.

Goldberg JL, Grant JWA, Lefebvre L. 2001. Effects of the temporal predictability and spatial clumping of food on the intensity of competitive aggression in the Zenaida dove. Behav Ecol. 12(4):490-495.

Goss-Custard JD, Clarke RT, Durell SEA. 1984. Rates of food intake and aggression of oystercatchers Haematopus ostralegus on the most and least preferred mussel Mytilus edulis beds on the Exe estuary. J Anim Ecol. 53:233-245.

Goymann W, East ML, Wachter B, Höner OP, Möstl E, Van't Hof TJ, Hofer H. 2001. Social, state-dependent and environmental modulation of faecal glucocorticoid levels in free-ranging female spotted hyenas. Proc R Soc Lond B Biol. 268:2453-2459.

Goymann W, Wingfield JC. 2004. Allostatic load, social status and stress hormones: the costs of social status matter. Anim Behav. 67:591-602. doi:10.1016/j.anbehav.2003.08.007.

Grafen A. 1984. Natural selection, kin selection and group selection. In: Krebs JR, Davies NB. Editors. Behavioural Ecology, 2nd edition. Oxford: Blackwell Scientific Publications. pp. $62-84$.

Grafen A. 1985. Hamilton's rule OK. Nature. 318:310-311.

Grafen A. 1987. The logic of divisively asymmetric contests: respect for ownership and the desperado effect. Anim Behav. 35:462-467. 
Grant JWA, Guha RT. 1993. Spatial clumping of food increases its monopolization and defense by convict cichlids, Cichlasoma nigrofasciatum. Behav Ecol. 4:293-296.

Green RH. 1979. Sampling Design and Statistical Methods for Environmental Biologists. New York: John Wiley.

Greengrass EJ. 2005. Mothers and offspring: social relationships and social behavior of the Kasekala community of chimpanzees at Gombe National Park, Tanzania. Ph.D. dissertation. Bristol: University of Bristol.

Greenwood PJ. 1980. Mating systems, philopatry and dispersal in birds and mammals. Anim Behav. 28:1140-1162.

Gruber T, Clay Z. 2016. A comparison between bonobos and chimpanzees: a review and update. Evol Anthropol. 25:239-252.

Grueter CC, Deschner T, Behringer V, Fawcett K, Robbins MM. 2014. Socioecological correlates of energy balance using urinary $C$-peptide measurements in wild female mountain gorillas. Physiol Behav. 127:13-19.

Grueter CC, Robbins AM, Abavandimwe D, Vecellio V, Ndagijimana F, Ortman S, Stoinski TS, Robbins MM. 2016. Causes, mechanisms, and consequences of contest competition among female mountain gorillas in Rwanda. Behav Ecol. 27:766-776.

Hackländer K, Möstl E, Arnold W. 2003. Reproductive suppression in female Alpine marmots, Marmota marmot. Anim Behav. 65:1133-1140.

Hamilton WD. 1964. The Genetical Evolution of Social Behaviour. I. J Theoret. Biol. 7:1-16.

Hand JL. 1986. Resolution of social conflicts: dominance, egalitarianism, spheres of dominance, and game theory. Q Rev Biol. 61:201-220.

Harcourt AH. 1987. Dominance and fertility among female primates. J Zool Lond. 213:471487. 
Harcourt AH. 1989. Environment, competition and reproductive performance of female monkeys. Trends Ecol Evol. 4:101-105.

Hare B, Melis AP, Woods V, Hastings S, Wrangham R. 2007. Tolerance allows bonobos to outperform chimpanzees on a cooperative task. Curr Biol. 17:619-623.

Hare B, Kwetuenda S. 2010. Bonobos voluntarily share their own food with others. Curr Biol. 20:230-231.

Hare B, Wobber V, Wrangham RW. 2012. The self-domestication hypothesis: evolution of bonobo psychology is due to selection against aggression. Anim Behav. 83:573-585.

Hare B, Yamamoto S. 2017. Bonobos: Unique in mind, brain, and behavior. Oxford: Oxford University Press.

Hare B. 2017. Survival of the friendliest: Homo sapiens evolved via selection for prosociality. Annu Rev Psychol. 68:155-186.

Harlow HJ, Miller B, Ryder T, Ryder L. 1985. Energy requirements for gestation and lactation in delayed implanter, the American badger. Comp Biochem Physiol. 82A:885-889.

Harper DGC. 1982. Competitive foraging in mallards: 'ideal free' ducks. Anim Behav. 30:575584.

Harris TR, Chapman CA, Monfort SL. 2010. Small folivorous primate groups exhibit behavioral and physiological effects of food scarcity. Behav Ecol. 21:46-56.

Hashimoto C, Takenaka O, Furuichi T. 1996. Matrilineal kin relationship and social behavior of wild bonobos (Pan paniscus): Sequencing the D-loop region of mitochondrial DNA. Primates. 37:305-318.

Hassell MP. 1975. Density-dependence in single-species populations. J Anim Ecol. 44:283295. 
Hauser B, Deschner T, Boesch C. 2008. Development of a liquid chromatography-tandem mass spectrometry method for the determination of 23 endogenous steroids in small quantities of primate urine. J Chromatogr B Biomed Sci Appl. 862:100-112.

Hauser MD. 1993. Primatology: some lessons from and for related disciplines. Evol Anthropol. 2(5):182-186.

Hawkley LC, Cole SW, Capitanio JP, Norman GJ, Cacioppo JT. 2012. Effects of social isolation on glucocorticoid regulation in social mammals. Horm Behav. 62(3):314-323.

Hedeskov CJ. 1980. Mechanism of glucose-induced insulin secretion. Physiol Rev. 60:442509.

Heesen M, Rogahn S, Ostner J, Schülke O. 2013. Food abundance affects energy intake and reproduction in frugivorous female Assamese macaques. Behav Ecol Sociobiol. 67:1053-1066.

Heesen M, Rogahn S, Macdonald S, Ostner J, Schülke O. 2014. Predictors of food-related aggression in wild Assamese macaques and the role of conflict avoidance. Behav Ecol Sociobiol. 68:1829-1841.

Heilbronner SR, Rosati AG, Stevens JR, Hare B, Hauser MD. 2008. A fruit in the hand or two in the bush? Divergent risk preferences in chimpanzees and bonobos. Biol Lett. 4:246-249.

Herman JP, Figueiredo H, Mueller NK, Ulrich-Lai Y, Ostrander MM, Choi DC, Cullinan WE. 2003. Central mechanisms of stress integration: hierarchical circuitry controlling hypothalamo-pituitary-adrenocortical responsiveness. Front Neuroendocrinol. 24:151-180.

Higham JP. 2016. Field endocrinology of nonhuman primates: past, present, and future. Horm Behav. 84:145-155.

Higham JP, Girard-Buttoz C, Engelhardt A, Heistermann M. 2011. Urinary C-peptide of insulin as a non-invasive marker of nutritional status: some practicalities. PLoS ONE 6(7):e22398. 
Hill RA, Lee PC. 1998. Predation risk as an infuence on group size in cercopithecoid primates: implications for social structure. J Zool Lond. 245:447-456.

Hinde K, Power ML, Oftedal OT. 2009. Rhesus macaque milk: Magnitude, sources, and consequences of individual variation over lactation. Am J Phys Anthropol. 138(2):148-157.

Hinde K, Milligan LA. 2011. Primate milk: proximate mechanisms and ultimate perspectives. Evol Anthropol. 20(1):9-23.

Hinde K, Skibiel AL, Foster AB, Del Rosso L, Mendoza SP, Capitanio JP. 2015. Cortisol in mother's milk across lactation reflects maternal life history and predicts infant temperament. Behav Ecol. 26(1):269-281.

Hinde RA. 1978. Dominance and role - two concepts with dual meanings.J Sociol Biol Struct. $1: 27-38$.

Hoffman CL, Ayala JE, Mas-Rivera A, Maestripieri D. 2010. Effects of reproductive condition and dominance rank on cortisol responsiveness to stress in free-ranging female rhesus macaques. Am J Primatol. 72(7):559-565. doi:10.1002/ajp.20793.

Hohmann G, Gerloff U, Tautz D, Fruth B. 1999. Social bonds and genetic ties: kinship, association, and affiliation in a community of bonobos (Pan paniscus). Behaviour 136:12191235.

Hohmann G, Fruth B. 2000. Use and function of genital contacts among female bonobos. Anim Behav. 60:107-120.

Hohmann G, Fruth B. 2002. Dynamics of social organization in bonobos (Pan paniscus). In: Boesch C, Hohmann G, Marchant LF, editors. Behavioural diversity in chimpanzees and bonobos. Cambridge: Cambridge University Press. p. 138-149.

Hohmann G, Fruth B. 2003. Lui Kotal - a new site for field research on bonobos in the Salonga National Park. Pan Afr News 10:25-27. 
Hohmann G, Mundry R, Deschner T. 2009. The relationship between socio-sexual behavior and salivary cortisol in bonobos: tests of the tension regulation hypothesis. Am J Primatol. $71: 223-232$.

Hohmann G, Potts K, N'Guessan A, Fowler A, Mundry R, Ganzhorn JU, Ortmann S. 2010. Plant foods consumed by Pan: Exploring the variation of nutritional ecology across Africa. Am J Phys Anthropol. 141:476-485.

Hood LC. 1994. Infanticide among ringtailed lemurs (Lemur catta) at Berenty Reserve, Madagascar. Am J Primatol. 33:65-69.

Houle A, Chapman CA, Vickery WL. 2007. Intratree variation in fruit production and implications for primate foraging. Int J Primatol. 28:1197-1217.

Houle A, Chapman CA, Vickery WL. 2010. Intratree vertical variation of fruit density and the nature of contest competition in frugivores. 64:429-441.

Houle A, Conklin-Brittain, NL, Wrangham RW. 2014. Vertical Stratification of the Nutritional Value of Fruit: Macronutrients and Condensed Tannins. Am J Primatol. 76:1207-1232.

Hrdy SB, Hrdy DB. 1976. Hierarchical relations among female Hanuman langurs (Primates: Colobinae, Presbytis entellus). Science. 193:913-915.

Isbell LA. 1991. Contest and scramble competition: patterns of female aggression and ranging behavior among primates. Behav Ecol. 2:143-155.

Isbell LA, Pruetz JD, Young TP. 1998. Movements of vervets (Cercopithecus aethiops) and patas monkeys (Erythrocebus patas) as estimators of food resource size, density, and distribution. Behav Ecol Sociobiol. 42:123-133.

Isbell LA, Young TP. 2002. Ecological models of female social relationships in primates: similarities, disparities, and some directions for future clarity. Behaviour. 139:177-202. 
Jacobson B, Grant JWA, Peres-Neto PR. 2015. The interaction between the spatial distribution of resource patches and population density: consequences for intraspecific growth and morphology. J Anim Ecol. 84:934-942.

Jaeggi AV, Stevens JMG, van Schaik CP. 2010. Tolerant food sharing and reciprocity is precluded by despotism among bonobos but not chimpanzees. Am J Phys Anthropol. 143:4151.

Janson CH. 2000. Primate socio-ecology: the end of a golden age. Evol Anthropol. 9:73-86.

Janson $\mathrm{CH}$, van Schaik CP. 1988. Recognizing the many faces of primate food competition: Methods. Behavior. 105:165-186.

Janson $\mathrm{CH}$, Chapman CA. 1999. Resources and primate community structure. In: Fleagle JG, Reed KE, editors. Primate communities. Cambridge: Cambridge University Press. p. 237-268.

Jarman PJ. 1974. The social organisation of antelope in relation to their ecology. Behaviour 48:215-267.

Jelincic JA, Tift MS, Houser DS, Crocker DE. 2017. Variation in adrenal and thyroid hormones with life-history stage in juvenile northern elephant seals (Mirounga angustirostris). Gen Comp Endocrinol. 252:111-118.

Jensen J, Rustad PI, Kolnes AJ, Lai YC. 2011. The role of skeletal muscle glycogen brekadown for regulation of insulin sensitivity by exercise. Front Physiol. 2(112):1-11.

Johnstone RA, Cant MA. 2010. The evolution of menopause in cetaceans and humans: the role of demography. Proc R Soc B, 277:3765-3771.

Jungers WL, Susman RL. 1984. Body size and skeletal anatomy in the African apes. In: Susman, RL, editor. The Pygmy Chimpanzee: Evolutionary Biology and Behavior. New York: Plenum Press. pp. 131-178. 
Kahlenberg SM. 2006. Female-female competition and male sexual coercion in Kanyawara chimpanzees. Ph.D. thesis. Harvard University, Cambridge, MA. pp. 61-62.

Kahlenberg SM, Emery Thompson M, Wrangham RW. 2008a. Female competition over core areas in Pan troglodytes schweinfurthii, Kibale National Park, Uganda. Int J Primatol. 29:931947.

Kahlenberg SM, Emery Thompson M, Muller MN, Wrangham RW. 2008b. Immigration costs for female chimpanzees and male protection as an immigrant counterstrategy to intrasexual aggression. Anim Behav. 76:1497-1509.

Kaneko T, Munemura M, Oka H, Oda T, Suzuki S. 1975. Demonstration of C-peptide immunoreactivity in various body fluids and clinical evaluation of the determination of urinary C-peptide immunoreactivity. Endocrinol Jpn. 22:207-212.

Kano T. 1980. Social behavior of wild pygmy chimpanzees (Pan paniscus) of Wamba: a preliminary report. J Hum Evol. 9:243-260.

Kano T. 1982. The social group of pygmy chimpanzees (Pan paniscus) of Wamba. Primates. 23(2):171-188.

Kano T. 1983. An ecological study of the pygmy chimpanzees (Pan paniscus) of Yalosidi, Republic of Zaire. Int J Primatol. 4(1):1-31.

Kano T, Mulawwa M. 1984. Feeding ecology of the pygmy chimpanzees (Pan paniscus) of Wamba. In: Susman, RL, editor. The Pygmy Chimpanzee: Evolutionary Biology and Behavior. New York: Plenum Press. pp. 233-274.

Kappeler PM, van Schaik CP. 2002. Evolution of primate social systems. Int J Primatol. 23:707-740.

Kaufmann JH. 1971. Is territoriality definable? In: Esser AH, editor. Behavior and environment: The use of space by animals and men. New York: Plenum Press. 
Kenagy GJ, Place NJ, Veloso C. 1999. Relation of glucocorticosteroids and testosterone to the annual cycle of free-living degus in semiarid central Chile. Gen Comp Endocrinol. 115:236-243.

Kenagy GJ, Place NJ. 2000. Seasonal changes in plasma glucocorticosteroids of free-living female yellow-pine chipmunks: Effects of reproduction and capture and handling. Gen Comp Endcrinol. 117:189-199.

King JA. 1973. The ecology of aggressive behavior. Ann Rev Ecol System. 4:117-138.

Kitaysky AS, Piatt JF, Hatch SA, Kitaiskaia EV, Benowitz-Fredericks ZM, Shultz MT, Wingfield JC. 2010. Food availability and population processes: severity of nutritional stress during reproduction predicts survival of long-lived seabirds. Func Ecol. 24:625-637.

Klass K, Cords M. 2011. Effect of unknown relationships on linearity, steepness and rank ordering of dominance hierarchies: Simulation studies based on data from wild monkeys. Behav Process. 88:168-176.

Kleiber M. 1975. Metabolic turnover rate: A physiological meaning of the metabolic rate per unit body weight. J Theor Biol. 53:199-204.

Koenig A. 2000. Competitive regimes in forest-dwelling Hanuman langur females (Semnopithecus entellus). Behav Ecol Sociobiol. 48:93-109.

Koenig A. 2002. Competition for resources and its behavioral consequences among female primates. Int J Primatol. 23:759-783.

Koenig A, Borries C, Chalise MK, Winkler P. 1997. Ecology, nutrition, and timing of reproductive events in an Asian primate, the Hanuman langur (Presbytis entellus). J Zool. 234:215-235.

Koenig A, Beise J, Chalise MK, Ganzhorn JU. 1998. When females should contest for food testing hypotheses about resource density, distribution, size, and quality with Hanuman langurs (Presbytis entellus). Behav Ecol Sociobiol. 42:225-237. 
Koenig A, Borries C. 2001. Socioecology of Hanuman langurs: The story of their success. Evol Anthropol. 10:122-137.

Koenig A, Borries C. 2006. The predictive power of socioecological models: A reconsideration of resource characteristics, agonism, and dominance hierarchies. In: Hohmann G, Robbins MM, Boesch C, editors. Feeding Ecology in Apes and Other Primates - Ecological, Physical, and Behavioral Aspects. Cambridge: Cambridge University Press. pp.263-284.

Koenig A, Borries C. 2009. The lost dream of ecological determinism: Time to say goodbye? ... Or a White Queen's proposal? Evol Enthropol. 18:166-174.

Koenig A, Scarry CJ, Wheeler BC, Borries C. 2013. Variation in grouping patterns, mating systems and social structure: what socio-ecological models attempt to explain. Phil. Trans. R. Soc. B. 368:20120348.

Koren L, Whiteside D, Fahlman Å, Ruckstuhl K, Kutz S, Checkley S, Dumond M, WynneEdwards K. 2012. Cortisol and corticosterone independence in cortisol-dominant wildlife. Gen Comp Endocrinol. 177:113-119.

Krieger DT, Allen W, Rizzo F, Krieger HP. 1971. Characterization of the normal temporal pattern of plasma corticosteroid levels. J Clin Endocrinol Metab. 32(2):266-284.

Kuo T, Harris CA, Wang JC. 2013. Metabolic functions of glucocorticoid receptor in skeletal muscle. Mol Cell Endocrinol. 380(1-2):79-88.

Kuroda S. 1979. Grouping of the pygmy chimpanzees. Primates 20:161-183.

Kuroda S. 1980. Social behavior of the pygmy chimpanzees. Primates. 21(2):181-197.

Kurta A, Bell GP, Nagy KA, Kunz TH. 1989. Energetics of pregnancy and lactation in freeranging little brown bats (Myotis lucifugus). Physiol Zool. 62:804-818.

Kuzuya T, Matsuda A. 1976. Disappearance rate of endogenous human C-peptide from blood. Diabetologia. 12(5):519-521. 
König B, Riester J, Markl H. 1988. Maternal care in house mice (Mus musculus): II. The energy cost of lactation as a function of litter size. J Zool. 216(2):195-210.

Landau HG. 1951a. On dominance relations and the structure of animal societies: I. Effect of inherent characteristics. Bull Math Biophys. 13:1-19.

Landau HG. 1951b. On dominance relations and the structure of animal societies: II. Some effect of possible social factors. Bull Math Biophys. 13:245-262.

Langergraber K. 2012. Cooperation among kin. In: Mitani JC, Call J, Kappeler PM, Palombit RA, Silk JB, editors. The Evolution of Primate Societies. Chicago and London: The University of Chicago Press. pp. 491-513.

Langergraber K, Mitani J, Vigilant L. 2009. Kinship and social bonds in female chimpanzees (Pan troglodytes). Am J Primatol. 71: 840-851.

Langergraber KE, Prüfer K, Rowney C, Boesch C, Crockford C, Fawcett K, Inoue E, InoueMuruyama M, Mitani JC, Muller MN, Robbins MM, Schubert G, Stoinski TS, Viola B, Watt D, Wittig RM, Wrangham RW, Zuberbühler K, Pääbo S, Vigilant L. 2012. Generation times in wild chimpanzees and gorillas suggest earlier divergence times in great ape and human evolution. Proc Natl Acad Sci USA 109:15716-15721.

Leakey JEA, Chen S, Manjgaladze M, Turturro A, Duffy PH, Pipkin JL, Hart RW. 1994. Role of Glucocorticoids and "Caloric Stress" in Modulating the Effects of Caloric Restriction in Rodents. Ann N Y Acad Sci. 719:171-194.

Lee Strier 2015. Complexities of undersanding female dispersal in primates. In: Furuichi T, Yawagiwa J, Aureli F, editors. Dispersing Primate Females. Tokyo: Springer Japan. pp.215231.

Lehmann J, Boesch C. 2009. Sociality of the dispersing sex: the nature of social bonds in West African female chimpanzees, Pan troglodytes. Anim Behav. 77:377-387.

Lewontin RC. 1961. Evolution and the Theory of Games. J Theor Biol. 1:382-403. 
Locati M, Lovari S. 1991. Clues for dominance in female chamois: Age, weight, or horn size? Aggress Behav. 17(1):11-15.

Lovari S, Rosto G. 1985. Feeding rate and social stress of female chamois foraging groups. In: Lovari S. editor. The Biology and Management of Mountain Ungulates. London: Croom Helm. pp. 102-105.

Lunn PG, Whitehead RG, Cole TJ, Austin S. 1979. The relationship between hormonal balance and growth in malnourished children and rats. $\mathrm{Br} \mathrm{J}$ Nutr. 41:73-84.

MacKinnon JR., MacKinnon KS. 1978. Comparative feeding ecology of six sympatric primates in West Malaysia. In: Chivers DJ, Herbert JA, editors. Recent Advances in Primatology, Vol. 1. London: Academic Press. pp. 305-321.

Majolo B, Lehmann J, de Bortoli Vizioli A, Schino G. 2012. Fitness-related benefits of dominance in primates. Am J Phys Anthropol. 147:652-660.

Malenky RK. 1990. Ecological factors affecting food choice and social organization in Pan paniscus. Ph.D. Thesis. SUNY at Stony Brook, NY.

Malenky RK, Stiles EW. 1991. Distribution of terrestrial herbaceous vegetation and its consumption by Pan paniscus in the Lomako Forest, Zaire. Am J Primatol. 23:153-169.

Malenky RK, Wrangham RW. 1994. A quantitative comparison of terrestrial herbaceous food consumption by Pan paniscus in the Lomako Forest, Zaire, and Pan troglodytes in the Kibale Forest, Uganda. Am J Primatol. 32:1-12.

Malenky RK, Kuroda S, Vineberg EO, Wrangham RW. 1994. The significance of terrestrial herbaceous foods for bonobos, chimpanzees, and gorillas. In: Wrangham RW, McGrew WC, de Waal FBM, Heltne PG, editors. Chimpanzee Cultures. Cambridge, Massachusetts: Harvard University Press. pp. 59-75.

Maynard Smith J. 1974. The theory of games and the evolution of animal conflicts. J. Theor. Biol. 47:209-221. 
Maynard Smith J. 1979. Game theory and the evolution of behaviour. Proc R Soc Lond B. 205:475-488.

Maynard Smith J. 1982. Evolution and the theory of games. Cambridge: Cambridge University Press.

Maynard Smith J, Price GR. 1973. The logic of animal conflict. Nature 246:15-18.

Maynard Smith J, Parker GA. 1976. The logic of asymmetric contests. Anim Behav. 24:159 175.

McCabe GM, Fedigan LM. 2007. Effects of reproductive status on energy intake, ingestion rates, and dietary composition of female Cebus capucinus at Santa Rosa, Costa Rica. Int J Primatol. 28:837-851.

McCabe GM, Emery Thompson M. 2013. Reproductive seasonality in wild Sanje mangabeys (Cercocebus sanjei), Tanzania: Relationship between the capital breeding strategy and infant survival. Behavior. 150:1399-1429.

McCabe GM, Fernandez D, Ehardt CL. 2013. Ecology of reproduction in Sanje mangabey (Cercocebus sanjei): Dietary strategies and energetic condition during a high fruit period. Am J Primatol. 75:1196-1208.

McCleery RH. 1977. On satiation curves. Anim Behav. 25:1005-1015.

McElreath R, Boyd R. 2007. Mathematical models of social evolution. Chicago: The University of Chicago Press. pp. 37-60.

McEwen BS. 1998. Stress, Adaptation, and Disease. Ann NY Acad Sci. 840:33-44.

McEwen BS, Wingfield JC. 2003. The concept of allostasis in biology and biomedicine. Horm Behav. 43:2-15. 
Messer NT, Johnson PJ, Refsal KR, Nachreiner RF, Ganjam VK, Krause GF. 1995. Effect of food deprivation on baseline iodothyronine and cortisol concentrations in healthy, adult horses. Am J Vet Re. 56:116-121.

Meunier J. 2015 Social immunity and the evolution of group living in insects. Phil. Trans. R Soc. B 370: 20140102. doi:10.1098/rstb.2014.0102.

Milinski M. 1979. An evolutionary stable feeding strategy in sticklebacks. Zietschrift für Tierpsychologie. 51:36-40.

Milinski M, Parker GA. 1991. Competition for resources. In: Krebs JR, Davies NB, editors. Behavioural Ecology: An Evolutionary Approach. Oxford: Blackwell Scientific Publications. p. 148-169.

Miller RS. 1967. Pattern and process in competition. Adv Ecol Res. 4:1-74.

Miller JA, Pusey AE, Gilby IC, Schroepher-Walke K, Markham AC, Murray CM. 2014. Competing for space: female chimpanzees are more aggressive inside than outside their core areas. Anim Behav. 87:147-152.

Mitchell CL, Boinski S, van Schaik CP. 1991. Competitive regimes and female bonding in two species of squirrel monkeys (Saimiri oerstedi and S. sciureus). Behav Ecol Sociobiol. 28:5560.

Monaghan P, Metcalf NB. 1985. Group foraging in wild brown hares: effects of resource distribution and social status. Anim Behav. 33:993-999.

Morin PA, Moore JJ, Chakraborty R, Jin L, Goodall J, Woodruff DS. 1994. Kin selection, social structure, gene flow, and the evolution of chimpanzees. Science 265:1193-1201.

Moscovice LR, Douglas PH, Martinez-Inigo L, Surbeck M, Vigilant L, Hohmann G. 2017. Stable and fluctuating social preferences and implications for cooperation among female bonobos at LuiKotale, Salonga National Park, DRC. Am J Phys Antropol. 163:158-172. 
Muller MN, Lipson SF. 2003. Diurnal patterns of urinary steroid excretion in wild chimpanzees. Am J Primatol. 60:161-166.

Muller MN, Wrangham RW. 2004. Dominance, cortisol and stress in wild chimpanzees (Pan troglodytes schweinfurthii). Behav Ecol Sociobiol. 55:332-340.

Muller MN, Kahlenberg SM, Emery Thompson M, Wrangham RW. 2007. Male coercion and the costs of promiscuous mating for female chimpanzees. Proc R Soc B. 274:1009-1014.

Murray CM, Eberly LE, Pusey AE. 2006. Foraging strategies as a function of season and rank among wild female chimpanzees (Pan troglodytes). Behav Ecol. 17:1020-1028.

Murray CM, Mane SV, Pusey AE. 2007. Dominance rank influences female space use in wild chimpanzees, Pan troglodytes: towards an ideal despotic distribution. Anim Behav. 74:17951804.

Murray CM, Lonsdorf EV, Eberly LE, Pusey AE. 2009. Reproductive energetics in free-living female chimpanzees (Pan troglodytes schweinfurthii). Behav Ecol. 20(6):1211-1216.

Muruthi P, Altmann J, Altmann S. 1991. Resource base, parity, and reproductive condition affect females' feeding time and nutrient intake within and between groups of a baboon population. Oecologia. 87:467-472.

Nakagawa N. 2009. Feeding rate as valuable information in primate feeding ecology. Primates. 50:131-141.

Narat V, Pennec F, Simmen B, Ngawolo JCB, Krief S. 2015. Bonobo habituation in a forestsavanna mosaic habitat: influence of ape species, habitat type, and sociocultural context. Primates. 56:339-349.

Newton PN. 1988. The variable social organization of Hanuman langurs (Presbytis entellus), infanticide, and the monopolization of females. Int J Primatol. 9:59-77.

Nicholson AJ. 1954. An outline of the dynamics of animal populations. Aust J Zool. 2:9-65. 
Nicholson AJ. 1957. The self-adjustment of populations to change. Cold Spring Harb Symp Quant Biol. 22:153-173.

Nurmi NO, Hohmann GH, Goldstone LG, Deschner T, Schülke O. 2018. Data from: The "tolerant chimpanzee" - towards the costs and benefits of sociality in female bonobos. Behavioral Ecology. Dryad Digital Repository. https://doi.org/10.5061/dryad.4c1246q

Olupot W, Chapman CA, Brown CH, Waser PM. 1994. Mangabey (Cercocebus albigena) population density, group size, and ranging: a twenty-year comparison. Am J Primatol. 32:197-205.

Ostner J, Schülke O. 2014. The evolution of social bonds in primate males. Behaviour 151:871-906.

Oyegbile TO, Marler CA. 2005. Winning fights elevates testosterone levels in California mice and enhances future ability to win fights. Horm Behav. 48:259-267.

Packer C, Scheel D, Pusey AE. 1990. Why lions form groups: food is not enough. Am Nat. 136:1-19.

Palme R, Rettenbacher S, Touma C, El-Bahr SM, Möstl E. 2005. Stress hormones in mammals and birds comparative aspects regarding metabolism, excretion, and noninvasive measurement in fecal samples. Ann NY Acad Sci. 1040:162-171.

Parish AR. 1994. Sex and food control in the "uncommon chimpanzee': how bonobo females overcome a phylogenetic legacy of male dominance. Ethol Sociobiol. 15:157-179.

Parish AR. 1996. Female relationships in bonobos (Pan paniscus): evidence for bonding, cooperation, and female dominance in a male-philopatric species. Hum Nat. 7:61:96.

Park T. 1954. Experimental studies of interspecies competition II. Temperature, humidity, and competition in two species of Tribolium. Phys Zool. 27(3):177-239.

Parker GA. 1970. The reproductive behaviour and the nature of sexual selection in Scatophaga stercoraria L. (Diptera: Scatophagidae). II. The fertilization rate and the spatial 
and temporal relationships of each sex around the site of mating and oviposition. J Anim Ecol. 39:205-228.

Parker GA. 1974a. The reproductive behaviour and the nature of sexual selection in Scatophaga stercoraria L. (Diptera: Scatophagidae). IX. Spatial distribution of fertilization rates and evolution of male search strategy within the reproductive area. Evolution. 28:93108.

Parker GA. 1974b. Assessment strategy and the evolution of fighting behavior. J Theor Biol. 47:223-243.

Parker GA. 2000. Scramble in behavior and ecology. Phil Trans R Soc Lond B. 355:16371645.

Parker GA, Sutherland WJ. 1986. Ideal free distributions when individuals differ in competitive ability: phenotype-limited ideal free models. Anim Behav. 34:1222-1242.

Parker GA, Maynard Smith J. 1990. Optimality theory in evolutionary biology. Nature. 348:2733.

Parker KL, Barboza PS, Gillingham MP. 2009. Nutrition integrates environmental responses of ungulates. Funct Ecol. 23:57-69.

Peckett AJ, Wright DC, Riddell MC. 2011. The effects of glucocorticoids on adipose tissue lipid metabolism. Metabolism. 60:1500-1510.

Plant TM. 1981. Time courses of concentrations of circulating gonadotropin, prolactin, testosterone, and cortisol in adult male rhesus monkeys (Macaca mulatta) throughout the 24 h light-dark cycle. Biol Reprod. 25:244-252.

Plat L, Byrne MM, Sturis J, Polonsky KS, Mockel J, Fery F, van Cauter E. 1996. Effects of morning cortisol elevation on insulin secretion and glucose regulation in humans. Am $J$ Physiol. 270(33):E36-E42. 
Plavcan JM, van Schaik CP, Kappeler PM. 1995. Competition, coalitions and canine size in primates. J Hum Evol. 28:245-276.

Pontzer H, Wrangham RW. 2004. Climbing and the daily energy cost of locomotion in wild chimpanzees: implications for hominoid locomotor evolution. J Hum Evol. 46:315-333.

Pontzer H, Raichlen DA, Gordon AD, Schroepfer-Walker KK, Hare B, O’Neill MC, Muldoon KM, Dunsworth HM, Wood BM, Isler K, Burkart J, Irwin M, Shumaker RW, Lonsdorf EV, Ross SR. 2013. Primate energy expenditure and life history. Proc Natl Acad Sci USA. 111:14331437.

Pradella M, Dorizzi RM, Rigolin F, Statland BE. 1988. Relative Density of Urine: Methods and Clinical Significance. Crit Rev Clin Lab Sci. 26(3):195-242.

Prado-Martinez J, Sudmant PH, Kidd JM, Li H, Kelley JL, Lorente-Galdos B, Veeramah KR Woerner AE, O'Connor TD, Santpere G, Cagan A, Theunert C, Casals F, Laayouni H, Munch K, Hobolth A, Halager AE, Malig M, et. al. 2013. Great ape genetic diversity and population history. Nature. 499:471-475.

Pride E. 2005. Foraging success, agonism, and predator alarms: Behavioral predictors of cortisol in Lemur catta. Int J Primatol. 26(2):296-319.

Pruetz JD, Isbell LA. 2000. Correlations of food distribution and patch size with agonistic interactions in female vervets (Chlorocebus aethiops) and patas monkeys (Erythrocebus patas) living in simple habitats. Behav Ecol Sociobiol. 49:38-47.

Prüfer K, Munch K, Hellmann I, Akagi K, Miller JR, Walenz B, Koren S, Sutton G, Kodira C, Winer R, Knight JR, Mullikin JC, Meader SJ, Ponting CP, Lunter G, Higashino S, Hobolth A, Dutheil J, Karakoç E, Alkan C, et. al. 2012. The bonobo genome comparedwith the chimpanzee and human genomes. Nature. 486:527-531.

Pschera H, Persson B, Lunell NO. 1986. Amniotic fluid C-peptide and cortisol in normal and diabetic pregnancies accompanied by fetal growth retardation. Am J Perinatol. 3:16-21. 
Pusey AE. 1990. Behavioural changes at adolescence in chimpanzees. Behaviour. 15:203246.

Pusey A. 2012. Magnitude and sources of variation in female reproductive performance. In: Mitani JC, Call J, Kappeler PM, Palombit RA, Silk JB, editors. The Evolution of Primate Societies. Chicago and London: The University of Chicago Press. pp. 343-366.

Pusey AE, Packer C. 1987. The evolution of sex-biased dispersal in lions. Behaviour. 101(4):275-310.

Pusey A, Williams J, Goodall J. 1997. The influence of dominance ank on the reproductive success of female chimpanzees. Science. 277:828-831.

Pusey AE, Murray CM, Wallauer W, Wilson M, Wroblewski EE. 2008. Severe aggression among female chimpanzees at Gombe National Park, Tanzania. Int J Primatol. 29:949-973.

Pusey AE, Schroepfer-Walker K. 2013. Female competition in chimpanzees. Phil Trans R Soc B 368: 20130077.

R Core Team (2017) R: A language and environment for statistical computing. R Foundation for Statistical Computing, Vienna, Austria. URL https://www.R-project.org/. (accessed 28 Feb 2017).

Rankin DJ. Kin selection and the evolution of sexual conflict. J Evol Biol. 24:71-81. doi: 10.1111/j.1420-9101.2010.02143.x.

Rimbach R, Link A, Montes-Rojas A, Di Fiore A, Heistermann M, Heymann EW. 2014. Behavioral and physiological responses to fruit availability of spider monkeys ranging in a small forest fragment. Am J Primatol. 76:1049-1061.

Robb SE, Grant JWA. 1998. Interactions between the spatial and temporal clumping of food affect the intensity of aggression in Japanese medaka. Anim Behav. 56:29-34. 
Robbins MM, Robbins AM, Gerald-Steklis N, Steklis HD. 2005. Long-term dominance relationships in female mountain gorillas: Strength, stability and determinants of rank. Behavior. 142:779-809.

Robinson KP, Sim TM, Culloch RM, Bean TS, Cordoba Aguilar I, Eisfeld SM, Filan M, Haskins GN, Williams G, Pierce GJ. 2017. Female reproductive success and calf survival in a North Sea coastal bottlenose dolphin (Tursiops truncatus) population. PLOS One. 12(9):e0185000.

Rodrigues MA, Wittwer D, Kitchen DM. 2015. Measuring stress responses in female Geoffroy's spider monkeys: Validation and the influence of reproductive state. Am J Primatol. 77(9):925-935.

Romero LM. 2004. Physiological stress in ecology: lessons from biomedical research. Trends Ecol Evol. 19:249-255.

Romero LM, Dickens MJ, Cyr NE. 2009. The reactive scope model - a new model integrating homeostasis, allostasis, and stress. Horm Behav. 55:375-389.

Russon AE, Begun DR. 2004. The Evolution of Great Ape Intelligence. Cambridge: Cambridge University Press.

Ruxton GD, Moody AL. 1997. The Ideal Free distribution with Kleptoparasitism. J Theor Biol. 186:449-458.

Sakamaki T, Ryu H, Toda K, Tokuyama N, Furuichi T. 2018. Increased frequency of intergroup encounters in wild bonobos (Pan paniscus) around the yearly peak in fruit abundance at Wamba. Int J Primatol. 1-20.

Salfen BE, Carroll JA, Keisler DH. 2003. Endocrine responses to short-term feed deprivation in weanling pigs. J Endocrinol. 178:541-551.

Saltzman W, Schultz-Darken NJ, Scheffler G, Wegner FH, Abbott DH. 1994. Social and reproductive influences on plasma cortisol in female marmoset monkeys. Physiol Behav. 4:801-810. 
Sapolsky RM. 1993. The physiology of dominance. In: Mason WA, Mendoza SP, editors. Primate Social Conflict. New York: State University of New York Press. pp. 171-204.

Sapolsky RM, Krey LC, McEwen BS. 1983. The adrenocorticol stress-response in the aged male rat: impairment of recovery from stress. Exp Gerontol. 18:55-64.

Sapolsky RM, Romero LM, Munck AU. 2000. How do glucocorticoids influence stress responses? integrating permissive, suppressive, stimulatory, and preparative actions. Endocr Rev. 21(1):55-89.

Sapolsky RM. 2005. The influence of social hierarchy on primate health. Science. 308:648652.

Schielzeth H. 2010. Simple means to improve the interpretability of regression coefficients. Meth Ecol Evol. 1:103-113.

Schielzeth H, Forstmeier W. 2009. Conclusions beyond support: overconfident estimates in mixed models. Behav Ecol. 20:416-420.

Schuett GW, Harlow HJ, Rose JD, Van Kirk EA, Murdoch WJ. 1996. Levels of plasma corticosterone and testosterone in male copperheads (Agkistrodon contortrix) following staged fights. Horm Behav. 30:60-68.

Schülke O, Chalise MH, Koenig A. 2006. The importance of ingestion rates for estimating food quality and energy intake. Am J Primatol. 68:951-965.

Schülke O, Ostner J. 2008. Male reproductive skew, paternal relatedness, and female social relationships. Am J Primatol. 70(7):695-698.

Schülke O, Ostner J. 2012. Ecological and Social Influences on Sociality. In: Mitani JC, Call J, Kappeler PM, Palombit RA, Silk JB, editors. The Evolution of Primate Societies. Chicago and London: The University of Chicago Press. pp. 195-219. 
Setchell JM, Smith T, Wickings EJ, Knapp LA. 2008. Factors affecting fecal glucocorticoid levels in semi-free-ranging female mandrills (Mandrillus sphinx). Am J Primatol. 70:10231032.

Sharpe RV, Avilés L. 2016. Prey size and scramble vs. contest competition in a social spider: implications for population dynamics. J Anim Ecol. 85:1401-1410.

Sherry DS, Ellison PT. 2007. Potential applications of urinary C-peptide of insulin for comparative energetics research. Am J Phys Anthropol. 133:771-778.

Shopland JM. 1987. Food quality, spatial deployment, and the intensity of feeding interference in yellow baboons (Papio cynocephalus). Behav Ecol Sociobiol. 21:149-156.

Silk JB. 2009. Nepotistic cooperation in non-human primate groups. Phil Trans R Soc B. 364: 3243-3254.

Slater KY, Schaffner CM, Aureli F. 2009. Sex differences in the social behavior of wild spider monkeys (Ateles geoffroyi yucatanensis). Am J Primatol. 71:21-29.

Smith CJ, Norman RL. 1987. Influence of the gonads on cortisol secretion in female rhesus macaques. Endocrinology. 121(6):2186-2191.

Smith LW, Link A, Cords M. 2008. Cheek pouch use, predation risk, and feeding competition in blue monkeys (Cercopithecus mitis stuhlmanni). Am J Phys Anthropol. 137:334-341.

Smith SM, Vale WW. 2006. The role of the hypothalamic-pituitary-adrenal axis in neuroendocrine responses to stress. Dial Clin Neurosci. 8:383-395.

Smith TE, French JA. 1997. Social and reproductive conditions modulate urinary cortisol excretion in black tufted-ear marmosets (Callithrix kuhli). Am J Primatol. 42:253-267.

Smuts BB, Smuts RW. 1993. Male aggression and sexual coercion of females in nonhuman primates and other mammals: Evidence and theoretical implications. Adv Study Behav. 22:163. 
Snaith TV, Chapman CA. 2005. Towards an ecological solution to the folivore paradox: patch depletion as an indicator of within-group scramble competition in red colobus monkeys (Piliocolobus tephrosceles). Behav Ecol Sociobiol. 59:185-190.

Sol D, Santos DM, Garcia J, Cuadrado M. 1998. Competition for food in urban pigeons: the cost of being juvenile. Condor 100:298-304.

Spellacy WN, Goetz FC. 1963. Plasma insulin in normal late pregnancy. New England J Med. 268:988.

Spellacy WN, Goetz FC, Greenberg BZ, Ells J. 1965. Plasma insulin in normal midpregnancy. Am J Obst Gynecol. 92:11-15.

Stearns FW. 2010. One hundred years of pleiotropy: a retrospective. Genetics. 186:767-773.

Stevens JMG, Vervaecke H, De Vries H, Van Elsacker L. 2006. Social structures in Pan paniscus: testing the female bonding hypothesis. Primates. 47:210-217.

Sterck EHM, Watts DP, van Schaik CP. 1997. The evolution of female social relationships in nonhuman primates. Behav Ecol Sociobiol. 41:291-309.

Stockley P, Bro-Jørgensen J. 2011. Female competition and its evolutionary consequences in mammals. Biol Rev. 86:341-366.

Strack AM, Sebastian RJ, Schwartz MW, Dallman MF. 1995. Glucocorticoids and insulin: reciprocal signals for energy balance.Am J Physiol. 268(1 Pt 2):R142-R149.

Strier KB. 1994. Myth of the typical primate. Yearb Phys Anthropol. 37:233-271.

Struhsaker TT. 1967. Social Structure among vervet monkeys (Cercopithecus aethiops). Behavior. 29:83-121.

Sugiyama Y. 1968. Social organization of chimpanzees in the Budongo forest, Uganda. Primates 9:225-258. 
Surbeck M., Mundry R, Hohmann G. 2011. Mothers matter! Maternal support, dominance status and mating success in male bonobos (Pan paniscus). Proc R Soc Lond B Biol. 278:590-598.

Surbeck M, Deschner D, Weltring A, Hohmann G. 2012. Social correlates of variation in urinary cortisol in wild male bonobos (Pan paniscus). Horm Behav. 62:27-35.

Surbeck M, Hohmann G. 2013. Intersexual dominance relationships and the influence of leverage on the outcome of conflicts in wild bonobos (Pan paniscus). Behav Ecol Sociobiol. 67:1767-1780.

Surbeck M, Deschner T, Behringer V, Hohmann G. 2015. Urinary C-peptide levels in male bonobos (Pan paniscus) are related to party size and rank but not mate competition. Horm Behav. 71:22-30.

Surbeck M, Boesch C, Girard-Buttoz C, Crockford C, Hohmann G, Wittig RM. 2017. Comparison of male conflict behavior in chimpanzees (Pan troglodytes) and bonobos (Pan paniscus), with specific regard to coalition and post-conflict behavior. Am J Primatol. 79:e22641.

Susman RL. 1984. The Pygmy Chimpanzee. Evolutionary Biology and Behavior. New York and London: Plenum Press.

Sussman RW. 1974. Ecological distinctions in sympatric species of Lemur. In: Martin RD, Doyle GA, Walker AC, editors. Prosimian Biology. London: Duckworth.

Sussman RW, Cheverud JM, Bartlett TQ. 1995. Infant killing as an evolutionary strategy: reality or myth? Evol Anthropol. 3:149-151.

Sussman RW, Garber PA, Cheverud JM. 2005. Importance of cooperation and affiliation in the evolution of primate sociality. Am J Phy Anthropol. 128:84-97.

Symington MM. 1988. Food competition and foraging party size in the black spider monkey (Ateles paniscus chamek). Behaviour. 105:117-134. 
Takasaki H. 1981. Troop size, habitat quality, and home range area in Japanese macaques. Behav Ecol Sociobiol. 9:277-281.

Takemoto H, Kawamoto Y, Furuichi T. How did bonobos come to range south of the Congo River? Reconsideration of the divergence of Pan paniscus from other Pan populations. Evol Anthropol. 24:170-184.

Taussky HH. 1954. A microcolometric determination of creatinine in urine by the Jaffe reaction. J Biol Chem. 208:853-862.

Terborgh J, Janson $\mathrm{CH}$. 1986. The socioecology of primate groups. Ann Rev Ecol Syst. 17:111-135.

Thierry B. 2007. Unity in diversity: Lessons from macaque societies. Evol Anthropol. 16:224238.

Thierry B. 2008. Primate socioecology, the lost dream of ecological determinism. Evol Anthropol. 17:93-96.

Thouless CR. 1990. Feeding competition between grazing red deer hinds. Anim Behav. 40:105-111.

Tokuyama N, Furuichi T. 2016. Do friends help each other? Patterns of female coalition formation in wild bonobos at Wamba. Anim Behav. 119:27-35.

Tokuyama N, Furuichi T. 2017. Leadership of old females in collective departures in wild bonobos (Pan paniscus) at Wamba. Behav Ecol Sociobiol. 71:55.

Tomiyama AJ, Mann T, Vinas D, Hunger JM, DeJager J, Taylor SE. 2010. Low Calorie Dieting Increases Cortisol. Psychosom Med. 72: 357-364.

Touma C, Palme R. 2005. Measuring fecal glucocorticoid metabolites in mammals and birds: The importance of validation. Ann NY Acad Sci. 1046:54-74. 
Townsend SW, Slocombe KE, Emery Thompson M, Zuberbühler K. 2007. Female-led infanticide in wild chimpanzees. Curr Biol. 17(10):R355-R356.

Tregenza T, Hack MA, Thompson DJ. 1996. Relative competitive success of unequal competitors changes with overall density. Oikos. 77:158-162.

Trivers RL. 1971. The evolution of reciprocal altruism. Quart Rev Biol. 46:35-57.

Trivers RL. 1972. Parental investment and sexual selection. In: Campbell B, editor. Sexual Selection and the Descent of Man, 1871-1971. Chicago: Aldine. pp.136-179.

Vagnuzzi AH. 1974. Time series analysis of hormonal patters in human plasma. Comput Biomed Res. 7:513-532.

VahI WK, Lok T, van der Meer J, Piersma T, Weissing FJ. 2005. Spatial clumping of food and social dominance affect interference competition among ruddy turnstones. Behav Ecol. 16:834-844.

Van Belle S, Estrada A. 2008. Group size and composition influence male and female reproductive success in black howler monkeys (Alouatta pigra). Am J Primatol. 70(6):613619.

van Cauter E, Leproult R, Kupfer DJ. 1996. Effects of gender and age on the levels and circadian rhythmicity of plasma cortisol. J Clin Endocrinol Metab. 81:2468-2473.

van Schaik CP. 1983. Why are diurnal primates living in groups? Behavior. 87:120-144.

van Schaik CP. 1989. Ecology of social relationships amongst female primates. In: Standen V, Foley RA, editors. Comparative Socioecology: the behavioural ecology of humans and other mammals. Oxford: Blackwell Scientific Publications. pp. 195-218.

van Schaik CP. 1996. Social evolution in primates: the role of ecological factors and male behavior. Proc. Br. Acad. 88:9-31. 
van Schaik CP, Noordwijk MA, van Bragt T, Blankenstein MA. 1991. A pilot study of the social correlates of levels of urinary cortisol, prolactin, and testosterone in wild long-tailed macaques (Macaca fascicularis). Primates 32:345-356.

Vasey N. 2004. Circadian rhythms in diet and habitat use in red ruffed lemurs (Varecia rubra) and white-fronted brown lemurs (Eulemur fulvus albifrons). Am J Phys Anthropol. 124:353363.

Vehrencamp SL. 1983. A model for the evolution of despotic versus egalitarian societies. Anim Behav. 31:667-682.

Vervaecke $\mathrm{H}$, de Vries $\mathrm{H}$, van Elsacker L. 1999. An experimental evaluation of the consistency of competitive ability and agonistic dominance in different social contexts in captive bonobos. Behaviour 136:423-442.

Vervaecke H, de Vries H, van Elsacker L. 2000. Dominance and its behavioral measures in a captive group of bonobos (Pan paniscus). Int J Primatol. 21:47-68.

Vogel ER. 2005. Rank differences in energy intake rates in white-faced capuchin monkeys, Cebus capucinus: the effect of contest competition. Behav Ecol Sociobiol. 58:333-344.

Vogel ER, Janson CH. 2007. Predicting the frequency of food-related agonism in white-faced capuchin monkeys (Cebus capucinus), using a novel focal-tree method. Am J Primatol. 69:533-550.

Vogel ER, Janson CH. 2011. Quantifying primate food abundance and distribution for socioecological studies: an objective consumer-centered method. Int J Primatol. 32:737-754.

Wade GN, Schneider JE. 1992. Metabolic Fuels and Reproduction in Female Mammals. Neurosci Biobeh Rev. 16:235-272.

Wakefield ML. 2013. Social dynamics among females and their influence on social structure in an East African chimpanzee community. Anim Behav. 85:1303-1313. 
Ward AJW, Webster MM, Hart PJB. 2006. Intraspecific food competition in fishes. Fish and Fisheries 7:231-261.

Weingrill T, Gray DA, Barrett L, Henzi PS. 2004. Fecal cortisol levels in free-ranging female chacma baboons: relationship to dominance, reproductive state and environmental factors. Horm Behav. 45:259-269.

Weingrill T, Willems EP, Zimmermann N, Steinmetz H, Heistermann M. 2011. Speciesspecific patterns in fecal glucocorticoid and androgen levels in zoo-living orangutans (Pongo spp.). Gen Comp Endocrinol. 172:446-457.

Wessling EG, Kühl HS, Mundry R, Deschner T, Pruetz JD. 2018a. The costs of living at the edge: Seasonal stress in wild savanna-dwelling chimpanzees. J Hum Evol. 121:1-11.

Wessling EG, Deschner T, Mundry R, Pruetz JD, Wittig RM, Kühl HS. 2018b. Seasonal variation in physiology challenges the notion of chimpanzees (Pan troglodytes verus) as a forest-adapted species. Front Ecol Evol. 6:60. doi: 10.3389/fevo.2018.00060.

West KL, Oftedal OT, Carpenter JR, Krames BJ, Campbell M, Sweeney JC. 2007. Effect of lactation stage and concurrent pregnancy on milk composition in the bottlenose dolphin. $J$ Zool. 273:148-160. doi:10.1111/j.1469-7998.2007.00309.x

West SA, Pen I, Griffin AS. 2002. Cooperation and competition between relatives. Science. 296:72-75.

White FJ. 1988. Party composition and dynamics in Pan paniscus. Int J Primatol. 9:179-193.

White FJ. 1989. Ecological correlates of pygmy chimpanzee social structure. In: Standen V, Foley RA, editors. Comparative Socioecology: the behavioural ecology of humans and other mammals. Oxford: Blackwell Scientific Publications. p. 151-164.

White FJ. 1996. Pan paniscus 1973 to 1996: twenty-three years of field research. Evol Anthropol. 5(1):11-17. 
White FJ. 1998. Seasonality and Socioecology: The importance of variation in fruit abundance to bonobo sociality. Int J Primatol. 19:1013-1027.

White FJ, Wrangham RW. 1988. Feeding competition and patch size in the chimpanzee species Pan paniscus and Pan troglodytes. Behaviour 105:148-163.

White FJ, Lanjouw A. 1992. Feeding competition in Lomako bonobos: Variation in social cohesion. In: Nishida T, McGrew WC, Marler P, Pickford M, de Waal FBM, editors. Topics in Primatology Vol I. Human Origins. Tokyo: University of Tokyo Press. pp. 67-79.

Whitten PL. 1983. Diet and dominance among female vervet monkeys (Cercopithecus aethiops). Am J Primatol. 5:139-159.

Wiens JA. 1976. Population responses to patchy environments. Ann Rev Ecol Syst. 7:81120.

Wilson ME, Moore CJ, Ethun KF, Johnson ZP. 2014. Understanding the control of ingestive behavior in primates. Horm Behav. 66:86-94.

Wilson ML, Wrangham, RW. 2003. Intergroup relations in chimpanzees. Annu. Rev. Anthropol. 32:363-392.

Wittig RM, Boesch C. 2003. Food competition and linear dominance hierarchy among female chimpanzees of the Taï National Park. Int J Primatol. 24:847-867.

Wittig RM, Crockford C, Weltring A, Deschner T, Zuberbühler K. 2015 .Single Aggressive Interactions Increase Urinary Glucocorticoid Levels in Wild Male Chimpanzees. PLoS ONE 10(2):e0118695. doi:10.1371/journal.pone.0118695.

Wittiger L, Boesch C. 2013. Female gregariousness in Western Chimpanzees (Pan troglodytes verus) is influenced by resource aggregation and the number of females in estrus. Behav Ecol Sociobiol. 67:1097-1111.

Wrangham RW. 1979. On the evolution of ape social systems. Social Science Information. 18:335-368. 
Wrangham RW. 1980. An ecological model of female-bonded primate groups. Behaviour 75:262-300.

Wrangham RW. 1986. Ecology and social evolution in two species of chimpanzees. In: Rubenstein DI, Wrangham RW, editors. Ecology and social evolution: birds and mammals. Princeton: Princeton University Press. p. 352-378.

Wrangham RW. 1993. The evolution of sexuality in chimpanzees and bonobos. Human Nature 4:47-79.

Wrangham RW. 2000. Why are male chimpanzees more gregarious than mothers? A scramble competition hypothesis. In: Kappeler PM, editor. Primate males: causes and consequences of variation in group composition. Cambridge: Cambridge University Press. p. 248-258.

Wrangham RW, Chapman CA, Clark AP, Isabirye-Basuta G. 1996. Social ecology of Kanyawara chimpanzees: implications for understanding the costs of great ape groups. In: McGrew WC, Marchant LF, Nishida T, editors. Great Ape Societies. Cambridge: Cambridge University Press: pp. 45-57.

Wrangham R, Pilbeam D. 2001. African apes as time machines. In: Galdikas BMF, Briggs NE, Sheeran LK, Shapiro GL, Goodall J. editors. All Apes Great and Small Volume I: African Apes. New York: Plenum Publishers. p. 5-17.

Wright E, Robbins AM, Robbins MM. 2014. Dominance rank differences in the energy intake and expenditure of female Bwindi mountain gorillas. Behav Ecol Sociobiol. 68:957-970.

Wright E, Grueter CC, Seiler N, Abavandimwe D, Stoinski TS, Ortmann S, Robbin MM. 2015. Energetic responses to variation in food availability in the two mountain gorilla populations (Gorilla beringei beringei). Am J Phys Anthropol. 158:487-500.

Young C, Majolo B, Heistermann M, Schülke O, Ostner J. 2014. Responses to social and environmental stress are attenuated by strong male bonds in wild macaques. Proc Natl Acad Sci USA. 111:18195-18200. 
Zinner D. 1999. Relationship between feeding time and food intake in hamadryas baboons (Papio hamadryas) and the value of feeding time as predictor of food intake. Zoo Biol. 18:495505.

Zucker EL, Clarke MR. 1998. Agonistic and affiliative relationships of adult female howlers (Alouatta palliata) in Costa Rica over a 4-year period. Int J Primatol. 19:433-449. 


\section{ACKNOWLEDGEMENTS}

The completion of my thesis would not have been possible without the guidance and support of my supervisors, mentors, funders, friends and family. Firstly, I am very grateful for my supervisors Oliver Schülke, Gottfried Hohmann and Tobias Deschner for giving me the opportunity to take on this project. I thank Oliver Schülke for encourament and guidance, which left me with a much improved understanding of the scientific method, thinking critically about results, and constructing a valid scientific argument. In addition, I am grateful to Oliver for the patience and perseverance, when I was not there to take advice in person and had to resort to written communications. I thank Gottfried Hohmann and Barbara Fruth for giving me the chance to work at LuiKotale, for their wonderful insights on bonobo behaviour and ecology, and for their kind support by providing advice on manuscripts, presentations, and bringing carepackages to the field. I thank Tobias Deschner for the guidance and great insights in to the physiology and endocrinology of great apes, which have left me with a great appreciation for the power of hormone analysis and rigorous laboratory methods.

I am very thankful to Hannes Rakoczy and Dietmar Zinner for their support as the members of my BeCog thesis committee; thank you for all the advice and enjoyable meetings, which really improved the structure of the project and made me think of it from a fresh angle. I am much obliged to Julia Ostner, Eckhard W. Heymann, Matthias Waltert, and Hannes Rakoczy for becoming members of my thesis examination board. I thank Clarissa Licht and Claudia Feige for their wonderful support with insurances, contracts, and travel arrangements for the fieldwork and beyond. I am grateful to Chris Schoegl for his advice during my studies. I also thank Rebecca Jürgens for support with the BeCog arrangements and while handing in my thesis. I thank Christophe Boesch for hosting me at the MPI EVA Leipzig, where I much benefited from the excellent facilities and support. I am very thankful to the Deutsche Forschungsgemeinschaft for providing funding for this project. In addition, I thank the Max Planck society for providing funds for the fieldwork costs.

My great gratitude to the Institut Congolaise pur la Conservation de la Nature for granting the permit to conduct fieldwork at LuiKotale and protecting the area with poaching patrols. I thank the villagers of Lompole for allowing me to experience their forest and their hospitability. My field seasons in DRC were only possible with the help of skillful, experienced colleagues. Matondi Mingi to Papa Endu, Fiston, Cistabol, Mangos, Lambert, Ndopo and Djaman for their support in the field. I thank Lucas Golstone for training me to identify and 
follow the bonobos, and for many fun field moments. I thank Josie Hubbard for company and endurance in the field, especially after having to depart the field momentarely due to an injury, much kudos for returning to LuiKotale and supporting me with further data collection! I thank Lieven Devreese for the interesting conversations and assistance in the field. Thanks to Karsten Dierks and Osamu Terao for making everything run smoothly at camp and for great company. I thank Tina Knittel for help with data entry, when I returned to Leipzig with all the handwritten notebooks. I further thank Vera Schmeling, Verena Behringer and Roisin Murtaugh for their excellent support with the hormone analyses. I thank Colleen Stephens and Roger Mundry for their training and support with R.

My deepest gratitude for the support and generosity of my extended family in Finland. I am greatly indebted to Erkki and Merja, without whom I would not have been able to complete this project. A big thanks my parents, sister and sister-in law for their loving, unwavering support throughout my studies. I thank my friends in Helsinki, Mari, Marsu, Minttu, Kirsi, Leena, Jaana, and Jenni, their patience and enduring friendship, even while I have spent prolonged periods away from Finland. A special thanks to Heidi and Biruk for all our shared moments!

I am thankful to Nga Nguyen and Peter Fashing for their friendship, support and mentoring. I further thank $\mathrm{Nga}$ and Peter for giving me the chance to work in their field site at Guassa, where I acquired field skills and indepence. I am grateful to Juha Merilä for providing advice and support during the most challenging phases of this thesis. I thank Abhilash Nair and Riitta Savolainen for great conservations and advice, which have greatly helped me during my studies. I thank John Deag for inspiration and advice during my B.Sc studies.

I thank my friends in Leipzig for their valuable support and company. Thanks to Mateja and Alex for their wonderful company as my trusted flatmates. I thank my great office mates Roisin and Alex for their company and advice. I thank Miri, Chris and Cedric for fun times and great ice-skating session. I much appreciate the support of Ed, Vroni, Laura, Haneul, Lauren, Patricia, Marie, Vidridge, Erin, and Stefano, a.k.a the coffee club; a cup of coffee and relaxing times were much needed to pace the work in the office. In addition, I thank Ed and Vroni for helpful comments on the manuscripts, and Vroni for help with the Zusammenfassung. Thank you also to Amy for many fun moments, especially raiding antique markets! I was fortunate to spend time also in Göttingen, where I much appreciated the company of Sally, Fred, Nadine, Marlies, Josie, Christine, Miranda, Andreas, Adeelia, Steffi, and Anni. A special thanks also to Anni, Steffi and Josie for offering their flats during my stays in Göttingen! 


\title{
CURRICULUM VITAE
}

\author{
NIINA ORVOKKI NURMI \\ Dept of Primatology $\cdot$ Max Planck Institute for Evolutionary Anthropology \\ Deutscher Platz 6 • 04103 Leipzig • Germany \\ e-mail: niina.nurmi@gmail.com
}

\section{EDUCATION}

2013 - $2018 \quad$ University of Göttingen, Ph.D. Behavioral Ecology, Göttingen, Germany Dissertation title: The tolerant chimpanzee - quantifying costs and benefits of sociality in wild female bonobos (Pan paniscus).

Committee: Oliver Schülke (Advisor), Dietmar Zinner, Hannes Rakozcy

$2010-2012 \quad$ University of Helsinki, M.Sc. Ecology \& Evolutionary Biology, 2012, Helsinki, Finland. Thesis: Lek dynamics and dispersal of capercaillie (Tetrao urogallus).

2004 - $2008 \quad$ University of Edinburgh, B.Sc. (Hons) Biology (Zoology), 2008, Edinburgh, UK. Honours Project: Terrestrial locomotion in birds.

\section{FIELD AND LABORATORY EXPERIENCE}

$2012-2014$

$2011-2012$

2011

$2008-2009$
LuiKotale Bonobo Research Project, DRC - Doctoral dissertation research on feeding competition in wild bonobos (18 months).

Molecular Ecology and Systematics Lab, University of Helsinki - M.Sc. research on patterns of genetic relatedness in wild capercaillie (7 months).

Ecological Genetics Research Unit, University of Helsinki - Research assistant for the Ph.D. projects of Jacquelin DeFaveri and Nurul Izza Ab Ghani on local adaptation in the three-spined sticklebacks (6 months).

Guassa Gelada Research Project, Ethiopia - Field manager of long-term research project on the wild geladas at Guassa (13 months).

\section{PUBLISHED PAPERS}

1) Nurmi, N.O., Hohmann, G., Goldstone, L.G., Deschner, T. \& Schülke O. (2018). The "tolerant chimpanzee" - towards costs and benefits of sociality in wild female bonobos. Behavioral Ecology

2) Nguyen, N., Lee, L.M., Fashing, P.J., Nurmi, N.O., Stewart, K.M., Turner, T.J., Barry, T.S., Callingham, K.R., Goodale, C.B., Kellogg, B.S., Burke, R.J., Bechtold, E.K., Claase, M.J., Eriksen, G.A., Jones, S.C.Z., Kerby, J.T., Kraus, J.B., Miller, C.M., Trew, T.H., Zhao, Y., Beierschmitt, E.C., Ramsay, M.S., Reynolds, J.D., \& Venkataraman, V.V. (2017). Comparative primate obstetrics: Observations of 15 diurnal births in wild gelada monkeys (Theropithecus gelada) and their implications for understanding human and nonhuman primate birth evolution. American Journal of Physical Anthropology DOI: 10.1002/ajpa.23141 


\section{PUBLISHED PAPERS (continued)}

3) Nguyen, N., Fashing, P.J., Boyd, D.A., Barry, T.S., Burke, R.J., Goodale, C.B., Jones, S.C.Z., Kerby, J.T., Kellogg, B.S., Lee, L.M., Miller, C.M., Nurmi, N.O., Ramsay, M.S., Reynolds, J.D., Stewart, K.M., Turner, T.J., Venkataraman, V.V., Knauf, Y., Roos, C. \& Knauf, S. (2015). Fitness impacts of tapeworm parasitism on wild gelada monkeys at Guassa, Ethiopia. American Journal of Primatology 77:579-594.

4) Fashing, P.J., Nguyen, N., Barry, T.S., Goodale, C.B., Burke, R.J., Jones, S.C.Z., Kerby, J.T., Lee, L.M., Nurmi, N.O., Venkataraman, V.V. (2011). Death among geladas (Theropithecus gelada): A broader perspective on mummified infants and primate thanatology. American Journal of Primatology 73: 405-409.

\section{MANUSCRIPTS IN PREPARATION}

1) Nurmi, N.O., Hohmann, G., Moscovice, L. Deschner, T. \& Schülke O. (in prep). Effects of reproductive state and dominance status on urinary cortisol levels in wild female bonobos ( $P a n$ paniscus).

\section{CONFERENCE TALKS \& POSTER PRESENTATIONS}

1) Nurmi, N.O., Hohmann, G., Goldstone, L.G., Deschner, T., Schülke O. (2018). The tolerant chimpanzee - towards costs and benefits of sociality in wild female bonobos. Poster at the 13th Meeting of the Ethologische Gesellschaft, Potsdam, Germany.

2) Nurmi, N.O., Hohmann, G., Goldstone, L.G., Deschner, T., Schülke O. (2017). The tolerant chimpanzee - towards cots and benefits of sociality in wild female bonobos. Talk at the 15th Meeting of the Gesellschaft für Primatologie, Zürich, Switzerland.

3) Nurmi, N.O., Gienapp, P., Merilä, J. (2012). Lek dynamics and dispersal of capercaillie (Tetrao urogallus). Poster at the Spring Symposium of the University of Helsinki, Finland.

4) Fashing, P.J., Nguyen, N., Kerby, J.T., Lee, L.M., Nurmi, N.O., and Venkataraman, V.V. (2010). Patterns and sources of mortality among geladas (Theropithecus gelada) at Guassa, Ethiopia. American Journal of Physical Anthropology S50:103.

5) Lee, L.M., Nurmi, N.O., Nguyen, N., and Fashing, P.J. (2010). Observations of multiple live births in wild geladas (Theropithecus gelada) at Guassa, Ethiopia. American Journal of Physical Anthropology S50:153.

6) Fashing, P.J., Nguyen, N., Kerby, J.T., Lee, L.M., Nurmi, N.O., Venkataraman, V.V. (2009). Two group takeovers, infanticides, and pregnancy terminations in Theropithecus gelada at Guassa, Ethiopia. Supplement to the American Association of Physical Anthropologists' meetings, Chicago, IL.

\section{MEDIA COVERAGE}

1) King, B.J. 2017. A new view into the primate birthing process. National Public Radio, February 2017.

2) Marshall, M. 2010. "Bereaved animals grieve - if their lifestyle allows it". New Scientist, issue 2791, December 2010. http://www.newscientist.com/article/mg20827913.900-bereavedanimals-grieve--if-their-lifestyle-allows-it.html 


\section{AWARDS}

2012

Winner of Best Poster of the Spring Symposium, University of Helsinki.

2011

Travel grant, Faculty of Biological and Environmental Sciences, University of Helsinki, for a course in Biotelemetry at UNIS Svalbard, Norway: $500 €$.

2011

Thesis grant, Ecological Genetics Research Unit, University of Helsinki: $1000 €$. 


\section{DECLARATION}

I hereby declare that I have written this thesis, entitled "The tolerant chimpanzee - quantifying costs and benefits of sociality in wild female bonobos (Pan paniscus), independently and with no other aids or sources than quoted.

Leipzig, 28.September 2018

Niina Nurmi 